\title{
An Exploration of Master's Degree Field Study and Teacher and Student Behavior in P.E
}

William J. Davis

Follow this and additional works at: https://researchrepository.wvu.edu/etd

\section{Recommended Citation}

Davis, William J., "An Exploration of Master's Degree Field Study and Teacher and Student Behavior in P.E" (2013). Graduate Theses, Dissertations, and Problem Reports. 7309.

https://researchrepository.wvu.edu/etd/7309

This Dissertation is protected by copyright and/or related rights. It has been brought to you by the The Research Repository @ WVU with permission from the rights-holder(s). You are free to use this Dissertation in any way that is permitted by the copyright and related rights legislation that applies to your use. For other uses you must obtain permission from the rights-holder(s) directly, unless additional rights are indicated by a Creative Commons license in the record and/ or on the work itself. This Dissertation has been accepted for inclusion in WVU Graduate Theses, Dissertations, and Problem Reports collection by an authorized administrator of The Research Repository @ WVU.

For more information, please contact researchrepository@mail.wvu.edu. 
An Exploration of Master's Degree Field Study and Teacher and Student Behavior in P.E. William J. Davis

Dissertation submitted to the College of Physical Activity and Sport Sciences at West Virginia University In partial fulfillment of the requirements for the degree of

Doctor of Philosophy

In

Kinesiology

Andrew Hawkins, Ph.D., Chair

Robert Wiegand, Ph.D. Sean Bulger, Ed.D.

Kristen Dieffenbach, Ph.D.

Deborah Hendricks, Ed.D.

Department of Physical Education

Morgantown, West Virginia

2013

Keywords: Expertise in P.E.; Field Systems Analysis; Master's Degree in P.E. Copyright 2013 William J. Davis 
UMI Number: 3571592

All rights reserved

INFORMATION TO ALL USERS

The quality of this reproduction is dependent upon the quality of the copy submitted.

In the unlikely event that the author did not send a complete manuscript and there are missing pages, these will be noted. Also, if material had to be removed, a note will indicate the deletion.

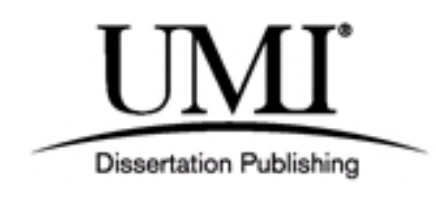

UMI 3571592

Published by ProQuest LLC (2013). Copyright in the Dissertation held by the Author.

Microform Edition () ProQuest LLC.

All rights reserved. This work is protected against unauthorized copying under Title 17, United States Code

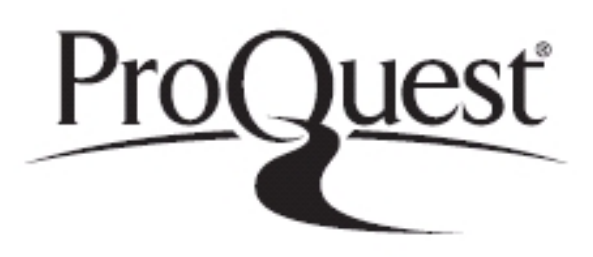

ProQuest LLC.

789 East Eisenhower Parkway

P.O. Box 1346

Ann Arbor, MI 48106 - 1346 


\section{ABSTRACT \\ An Exploration of Master's Degree Field Study and Teacher and Student Behavior in P.E.}

\section{William J. Davis}

This study analyzed two teacher and their corresponding student behavioral systems to determine whether discipline-specific ongoing education may play a role in gaining expertise in physical education. Field systems analysis (FSA) was used to analyze an all-encompassing categorical system, which was created by induction and then used to deductively analyze the two teachers and their corresponding students. Interview data were used as well, in conjunction with FSA, to understand more completely what role each teacher's ongoing education may have played in their behavioral systems. Both teachers received training from the same undergraduate physical education program and were of the same gender and had the same years of teaching experience. These two teachers taught the same content to the same number of students in one physical education lesson. The results exhibited differences in each teacher's behavioral system, as the teacher with ongoing education within-field exhibited a higher percentage of instructional elements that were more common, complex and predictable in their elemental chains. The teacher who received ongoing education out-of-field exhibited more managerial elements in their behavior system, while exhibiting less common, complex and predictable elemental chains. The students' data exhibited much of the same phenomenon, as the students who were taught by the within-field teacher exhibited more content learning elements that were more common, complex and predictable in their elemental chains. The students who were taught by the out-of-field teacher exhibited more non-content learning elements, while exhibiting less common, complex and predicable elemental chains. The interview data paralleled the behavioral systems for each teacher, as the within-field teacher revealed an ongoing education tailored to delivering physical education content to students, while the out-of-field teacher revealed how his ongoing education dealt with more managerial concerns. 


\section{Table of Contents}

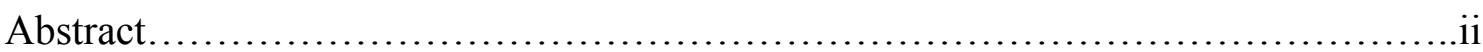

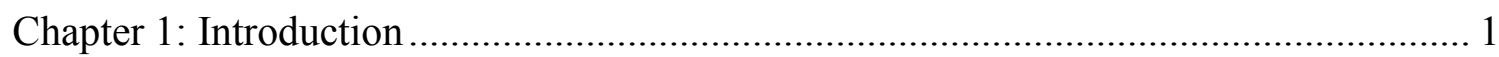

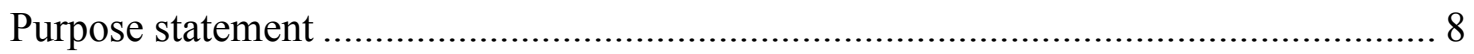

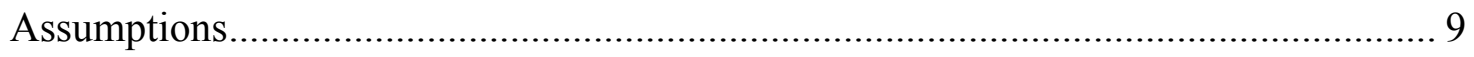

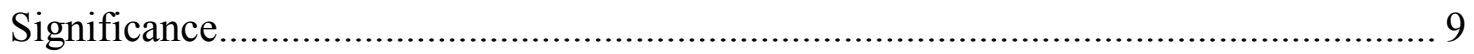

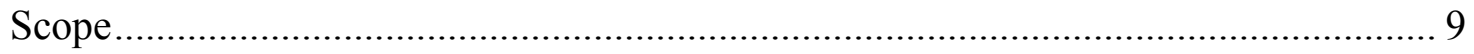

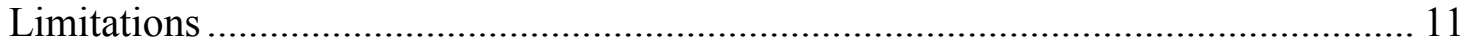

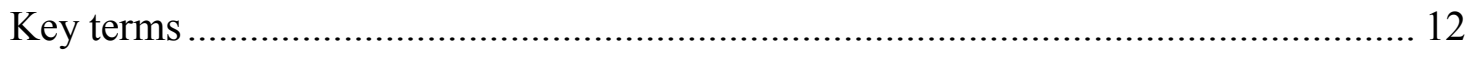

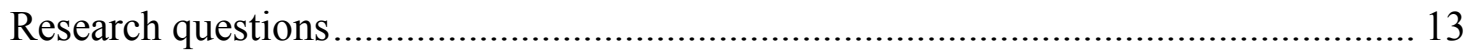

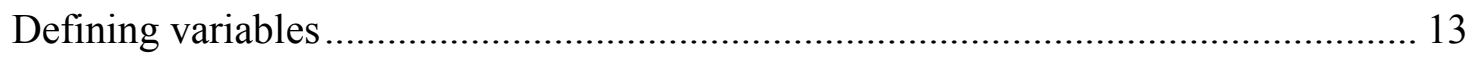

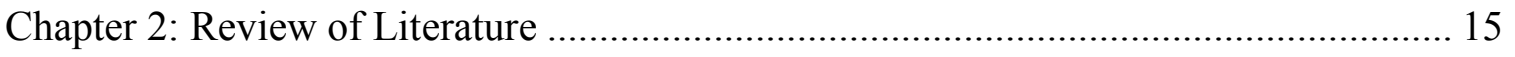

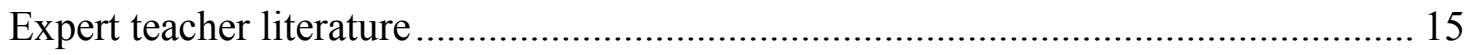

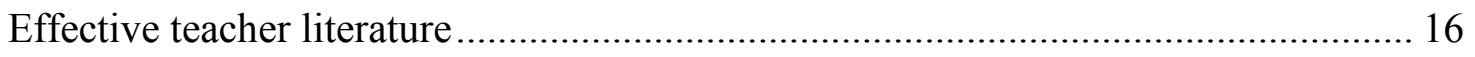

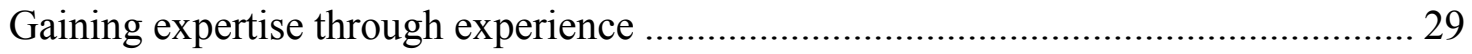

Ambivalence in gaining expertise through continuing education............................. 37

Master's degree out-of-field literature ........................................................ 37

Master's degree within-field literature ................................................... 40

Measurement of effectiveness and expertise ................................................... 44

Effectiveness evaluation tools............................................................. 44

Expertise evaluation tool ambivalence ….................................................. 45

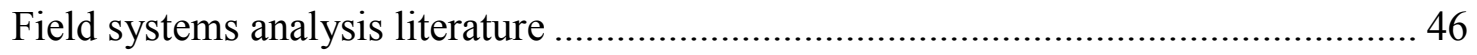




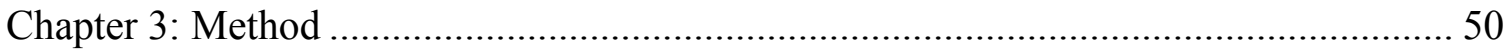

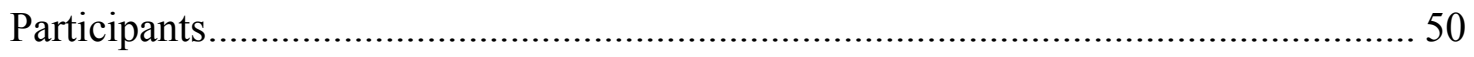

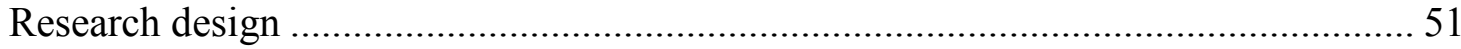

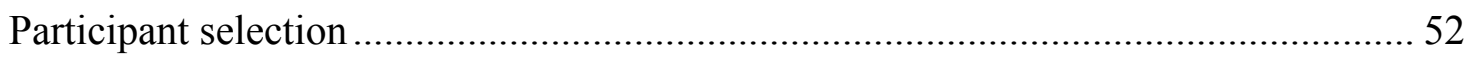

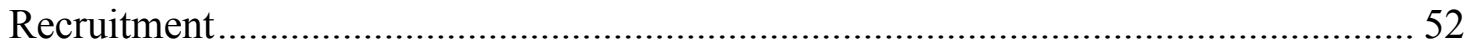

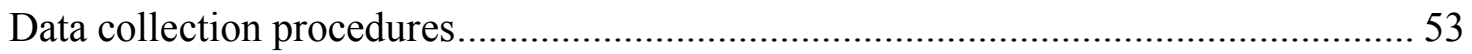

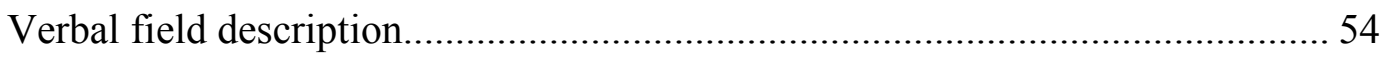

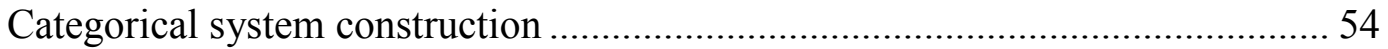

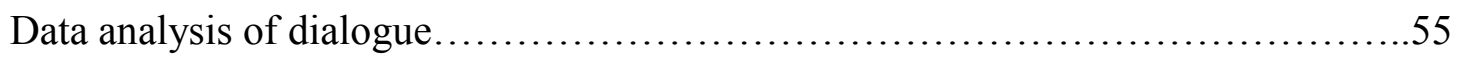

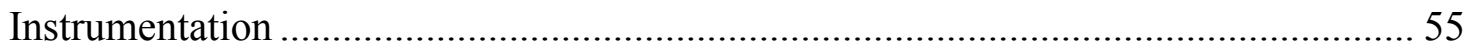

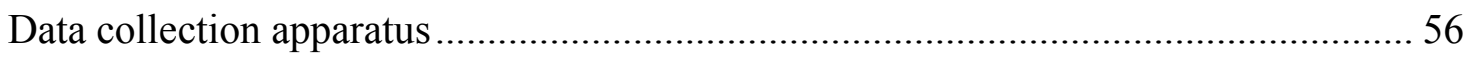

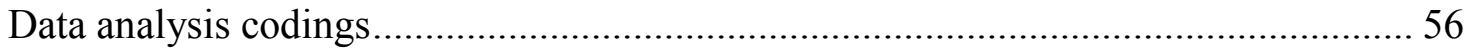

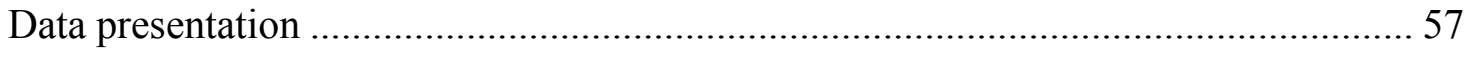

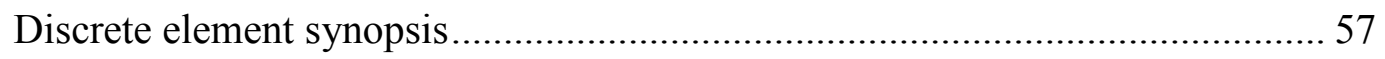

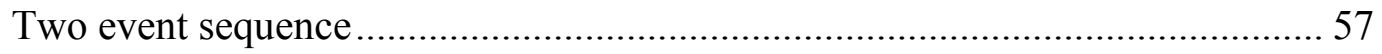

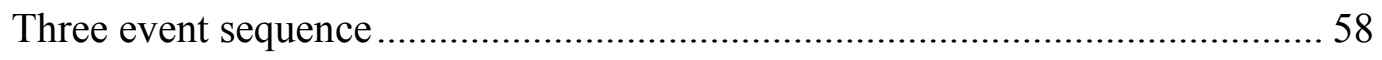

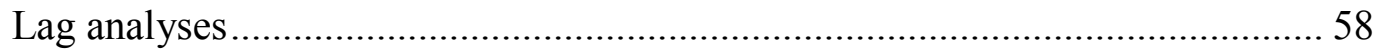

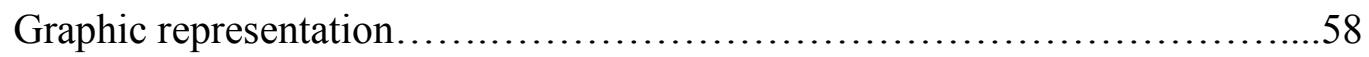

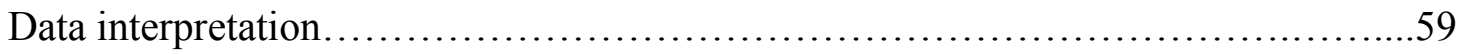

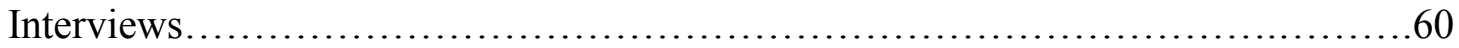

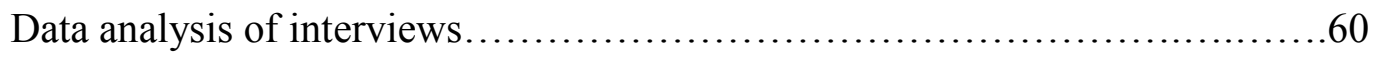

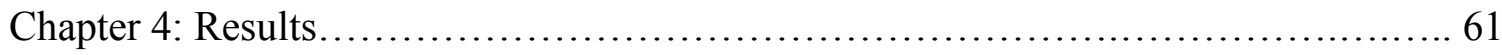


Teacher/subject contextual information....................................61

Environmental and behavioral category system teacher.........................62

Environmental and behavioral category system student........................64

Descriptive differences teachers....................................... 65

Summary of teacher behavior..................................... 70

Descriptive differences students....................................... 71

Summary of student behavior......................................75

Sequence differences teachers......................................... 75

Two event sequence.............................................75

In summation.................................................

Three event sequence................................................ 83

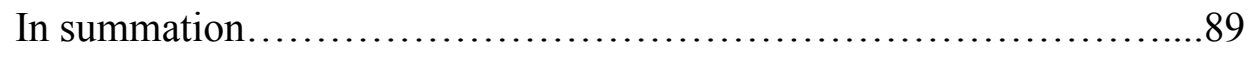

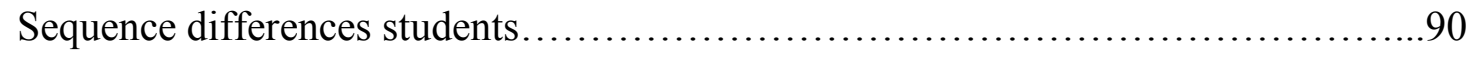

Two event sequence.............................................90

In summation........................................... 96

Three event sequence............................................97

In summation............................................. 100

Graphical data......................................................... 100

Teachers........................................................100

In summation............................................... 107

Graphical data......................................................... 108

Students......................................................... 108

In summation............................................. 112 
Data interpretation..................................................... 113

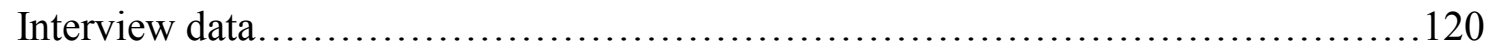

Descriptive, sequence and interview data.................................. 128

Chapter 5: Discussion.................................................. 130

A behavioral explanation................................................130

Teachers.......................................................... 130

Students................................................................ 134

Expertise revisited.................................................... 136

Research implications..................................................136

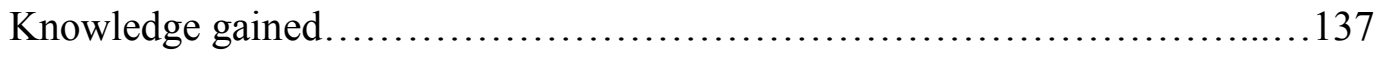

Future studies.........................................................139

Teacher development.............................................139

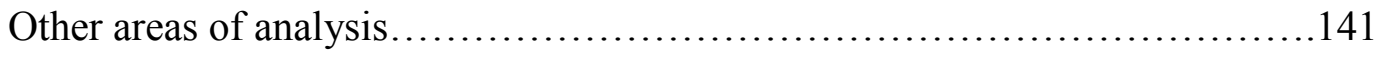

Strengthening future studies....................................... 141

References............................................................ 143

Appendix A.............................................................. 152

Appendix B........................................................... 162

Appendix C........................................................... 167

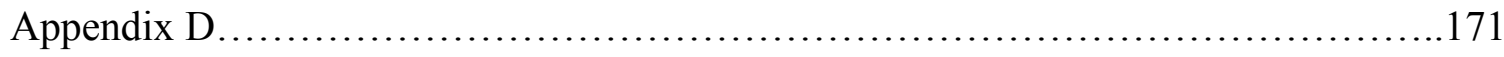

Appendix E.......................................................... 174

Appendix F............................................................. 179 


\section{CHAPTER 1}

\section{Introduction}

Expertise in teaching and the relationship between teacher behavior, student learning and the relevance of master's level education have been topics of interest among the educational community as of late. As school districts are increasingly examining effective practice based on student achievement, a characteristic that may lead to expertise, and the public is increasingly adamant about the idea of teacher pay being tied to accountability, it is essential that an examination of ongoing education and the specificity of a master's degree are evaluated (Johnson, 2000). Recently, teacher effectiveness and accountability have been stressed throughout the No Child Left Behind Act (NCLB) of 2001 and have addressed the issue about what constitutes a highly qualified teacher (U.S. Department of Education, 2004). Research findings have led to mixed messages about whether highly qualified teachers, ones who have received ongoing education amongst other things, are deemed more effective than less educated teachers. With more emphasis being placed on "core" math and reading coursework in the NCLB legislation, effective practice in the physical education environment must be achieved and maintained to receive ongoing curricular relevance (Pate et al., 2006; Siedentop, 2009).

Gaining expertise in the teaching field has been described to occur in different ways. Some researchers have provided a host of characteristics to encompass this term "expert," while others cite more subtle behavioral interactions of teachers and students to amass such a distinction (Berliner, 1986; Dodds, 1994, Sharpe \& Hawkins, 1992a; van der Mars, H., Darst, P.W., Vogler, E.W., \& Cusimano, B., 1995). Berliner (1986) has cited characteristics such as: (a) state teacher of the year, (b) principal selection, (c) mentorship, (d) officer within the 
discipline, and (e) teaching effectiveness, as components that may lead to greater expertise. Dodds (1994) has cited: (a) proper attitudes and beliefs, (b) content knowledge, and (c) maximization of student learning as characteristics describing qualities that may contribute to this definition. Research by van der Mars et al. (1995) has stated that expertise can be a relative term consisting of many factors including teaching (a) experience, (b) effectiveness, and (c) highly skilled teaching exhibition. Sharpe and Hawkins (1992a) have suggested that behavioral interactions of teachers, which occur subtly, may determine more expert practice.

Teacher effectiveness within the classroom has been defined as student learning relevant to grade and ability level with a value added to this process (The Center for Public Education, 2005). Effectiveness can be measured by teacher behaviors in sequence and isolated student behavior that may lead to student achievement gains (Hawkins, Wiegand, \& Bahneman., 1983; McKenzie, 2009). Effectiveness in the physical education environment is reflective of Academic Learning Time in Physical Education (ALT-PE) which is defined as a student's engaged time spent in skill practice in which the student performs with a high degree of success (Parker, 1989).Teaching effectiveness has been described as being only one behavioral indicator of expertise (Schempp et al., 2004). This denotes the utmost inclusion of effectiveness in attaining teaching expertise, but not the sole component necessary for expertise acquisition.

Research has suggested that one way expertise can be attained is through years of experience. In an examination of experienced versus inexperienced teachers in physical education, experienced teachers (a) exhibited more instructional strategies, (b) provided more individualized attention, and (c) gave more skill related statements (Housner \& Griffey, 1985). Along the same lines, Siedentop and Eldar's (1989) article examining expertise, experience and effectiveness, suggested that experienced teachers (a) reinforced correct student behaviors 
intentionally, (b) used time more efficiently, (c) exhibited less confusion, and (d) reflected higher levels of student engagement than their novice counterparts. Griffey and Housner's (1991) study examining experienced versus inexperienced teachers suggested that experienced teachers (a) gave more information within their class, (b) made decisions more quickly, (c) provided more specific observation, and (d) made more skill related statements. These studies delineated qualitative descriptions of what occurred in each environment, which may lead to gains in expertise. Regarding greater amounts of ALT-PE, however, mixed messages were discovered regarding experienced versus novice teachers (Al-Mulla, 2002; van der Mars et al., 1995; Vogler, van der Mars, Cusimano, \& Darst, 1992.) In each of these studies analyzing experienced teachers, similar yet differing criteria were used to define the more expert pedagogue.

Along with experience, another way of attaining expertise in teaching may be through continuing education. Increasingly more states, including West Virginia, are requiring teachers to obtain a master's degree to receive permanent certification (U.S. Department of Education, 2011). However, this education does not have to be discipline specific relative to one's undergraduate education. The ongoing education can be in another discipline, in administration, or in any field pertaining to education, and can even be obtained online. In fact, upwards of $90 \%$ of teachers' master's degrees are completed outside the subject area of which they teach (Terry, 2009). Based on this ambiguity relating to ongoing education, one wonders what part it may play, if any, in gaining expertise in one's teaching field.

Regarding fields other than physical education, research findings have shown interesting results regarding ongoing education and its part in gaining expertise. Other disciplines, however, have only examined teacher effectiveness, which is a necessary component in developing expertise, but not the sole determinant as cited previously. When examining teacher effectiveness 
from the standpoint of educational preparation, studies examining master's degrees in terms of teaching effectiveness have yielded interesting results. A Texas School Project Data study in the elementary setting cited no differences in teaching effectiveness when it examined pre and post test scores of students in teachers classrooms who held bachelor degrees in the field they taught and held master's degrees out-of-field, compared to those teacher's who held only bachelor's degrees within their field (Rivkin, Hanushek, \& Kain, 2005). Another study that examined student achievement within 4,000 elementary school teachers' classrooms resulted in a negative effect on student learning for teachers having a master's degree out-of field, as opposed to teachers who held only a bachelor's degree in-field (Clotfelter, Ladd \& Vidgor, 2007). In the area of receiving advanced education in-field versus out-of-field, research by Harris and Sass (2007) suggested that having a master's degree out-of-field did not contribute to teacher effectiveness relative to student achievement in any area, with the exception of middle school math. Financial incentive to obtain any master's degree has often times been the driving factor, not increasing the effectiveness of instruction (Terry, 2009).

An examination of teacher effectiveness pertaining to within-field master's degrees in other fields has yielded more optimistic results. Goldhaber and Brewer's (2000) results suggested greater teacher effectiveness gains relative to student achievement in math when the teacher had a master's degree within-field when compared to a teacher having an out-of-field advanced degree. Similarly, Betts, Zau and Rice (2003) concluded that differences existed only in student achievement in the reading discipline if teachers held a master's or doctorate in their field, citing the importance of a within-field advanced degree. In an analysis of master's degrees and teaching effectiveness by Roza and Miller (2009), strong differences existed between within and out-of-field advanced education. It was determined that obtaining master's degrees out-of- 
field yielded no correlation to increases in teaching effectiveness. Master's degrees in math, once again, revealed teaching effectiveness gains if individuals held undergraduate degrees in the same discipline. Even in the field of science, ongoing science training increased student achievement, because the education was discipline specific. This research suggests, as in other disciplines, master's degrees within-field have shown promising results in regards to more effective practice. This discipline-specific master's degree research, citing teacher effectiveness gains, suggests specific ongoing education may contribute to expertise. Based on these fields other than physical education, effective practice differences have been cited when individuals have obtained master's within-field as opposed to out-of-field. Even in these other fields, however, expertise was not analyzed.

Research regarding ongoing education and its relationship to gaining expertise in physical education has been non-existent. A possible reason for this absence in research regarding ongoing education and its role in gaining expertise in teaching in physical education, and in other fields, has been the lack of attention given to the systemic nature of instruction-incontext (Sharpe \& Hawkins, 1992a).

Examining variables that constitute teaching effectiveness has been a far more easily described avenue of focus. Tools evaluating effective instruction include ones such as the West Virginia University Teaching Evaluation System (WVUTES) and The System for Observing Fitness Instruction Time (SOFIT). These have been created to evaluate teacher and student behaviors in a linear nature (Hawkins et al., 1983; McKenzie, 2009). These tools can evaluate the frequency and duration of deductively created elements occurring in a lineal nature relating to effectiveness. Regarding effectiveness evaluations, examining isolated components, or 
behaviors, based on previously designed systematic evaluation tools can descriptively detail differences between effective and ineffective instructors (Sharpe \& Hawkins, 1992a).

Descriptive differences in student and teacher behaviors and qualitative analyses have been studied to determine what constitutes teaching effectiveness. However, complex and subtle factors which may lead to what constitutes teaching expertise are a far less common avenue of focus (Dawe, 1984; Eisner, 1983; Gage, 1978; Rubin, 1985; Sharpe \& Hawkins, 1992a).

One reason for the neglect of examining expertise may be that identifying, defining and measuring far more variables than the previously determined ones is a more arduous and time intensive process which involves induction and proper communication in dissemination of results (Hawkins, 1992). In examining interactions taking place, a richer description of factors that may contribute to expert practice may be determined. These intricacies such as the temporal locus of behavior, the temporal extent of the behavior, the context where the behavior occurs, and the multiple interactions among the many stimuli and behaviors occurring, gives a richer description which has been previously under-examined (Sharpe \& Hawkins, 1992a). Previously determined category systems may lack sensitivity to factors which may measure expertise in teaching. Some of these factors that may be attributed to differences in an individual's behavioral system may be related to the nature of an individual's advanced training, which previous literature in other disciplines has suggested (Betts et al., 2003; Goldhaber \& Brewer, 2000; Roza \& Miller, 2009). An analysis that takes into consideration the content, context, subject, and multiple stimuli and behaviors occurring simultaneously can more holistically determine the non-linear interactions taking place to examine what may be missed in a typical cause-effect study (Delprato, 1992). In an examination of these elements and their interactions, subtle differences which may occur in an expert teacher's instruction can be further understood. In 
regards to this, ongoing education and its significance in gaining expertise in physical education can be examined relative to one's behavioral system.

Field systems analysis (FSA) has been an area of research that examines what actually occurs in an environment. FSA is defined as a way of describing multiple concurrent factors in real time (Delprato, 1992). Behaviors which occur relative to a systematic phenomenon may not occur linearly and aspects of it can occur concurrently. These behaviors that affect each other to some degree may not occur in a clean manner reflected by the linear nature of the stimuliresponse-consequence (S-R-C) pathway. FSA examines how natural events occur as integrated wholes and not independent parts pieced together. In this manner, multiple behaviors occurring simultaneously can be analyzed to determine what interactions are occurring in an environment over time. With this holistic analysis of behaviors occurring simultaneously, one can examine the relationships that occur, which otherwise may have been hidden through systematic linear analysis of behavior. In this form of behavioral analysis, many behavioral variables that occur simultaneously can be evaluated to determine if otherwise hidden interactions taking place constitute more expert practices in the instructional setting. Clearly defined behavioral chains and temporal immediacy of such chains have been cited as more effective teaching practices in the physical education environment (Sharpe, Hawkins \& Wiegand, 1989). FSA examines a combination of these teaching behaviors including instructionally significant chains occurring simultaneously which can result in greater understanding of what expertise encompasses (Thaxton, Rothstein \& Thaxton, 1977). In analyzing these behavioral systems from the standpoint of elemental chains relative to the actual field data exhibited, an authentic, fieldspecific output can determine more complex interactions taking place. In an examination of these interactions, the behavioral systems may distinguish experts from non-experts. 
A study using FSA measuring expertise regarding experienced versus novice instruction was accomplished by Sharpe and Hawkins (1992b). This study examined differences in two teachers' behavioral systems, their settings and other intricate factors in conjunction. In this study, one instructor who had more years of experience and was cited as being a "teacher of the year" award winner, showed more expertise in her behavioral system regarding dimensional data. This individual had a higher rate of change in her behavior while maintaining higher probability and coherence in the behaviors that followed the initial triggers. This individual exhibited more complexity in her behavioral chains while maintaining coherence relative to predictability. This study suggested that years of experience amongst other things may have contributed to gains in expertise.

Gaining expertise through experience has been an avenue of focus among researchers in the physical education environment, examining ongoing education and its relationship to gaining expertise has not been examined up until this study. FSA has been used in this study to determine differences in the behavioral systems of master's educated teachers, ones who have received ongoing education within or out-of-field. This study has been a replication of the Sharpe and Hawkins (1992b) study examining one expert and one novice instructor, insofar as to examine the role ongoing education has possibly played in developing expertise.

\section{Purpose statement:}

The purpose of this study was to examine the complexities of two teacher's behavioral systems regarding dimensional data which may relate to their differences in ongoing education. This study also examines the complexities of these two teacher and corresponding student behavioral systems. 


\section{Assumptions:}

1. The two physical educators in the study have had similar demographic backgrounds and have been subjected to similar instructional settings as they teach the same lesson content.

2. Master's degree type have played a part in their behavioral systems

\section{Significance}

Examining teacher effectiveness in relation to master's degree education has been examined in other disciplines such as math, science, and reading along with elementary education. Specifically, teacher effectiveness, in regards to student achievement has come into focus due to the costs of ongoing education and ineffectiveness that has been documented from obtaining a master's degree out-of-field (Harris \& Sass, 2007). Examining the role experience plays in developing expertise has been studied by Sharpe and Hawkins' (1992b) FSA study. Examining teacher behavior and resultant student behavior interactions in relation to a subject matter specific master's or non-subject matter specific master's degree has not been undertaken to date in the physical education environment up until this specific study. This specific study has used a case study approach to examined two individuals who have master's degrees within or out-of-field along with other very similar characteristics. This study has evaluated each teacher's behavioral system for systemic differences in relation to the ongoing education they have received. This study has also examined these teacher's student's behavioral systems.

\section{Scope}

Participants in this study were selected based on the criteria of having five or more years of teaching experience in physical education after their bachelor's degree in physical education. These individuals have both attained a bachelor's degree in physical education from the same 
standardized physical education teacher education program. One of the two participants has attained a master's degree in physical education and the other individual has attained a master's degree in administration. Five or more years of teaching experience for each teacher constitutes each as having experience, as cited in previous literature (Griffey \& Housner, 1991). Each individual in this study has exactly five years of experience teaching physical education.

These two participants $(n=2)$ in this study have been selected on a volunteer basis. One has earned a within-field masters, and one has earned an out-of-field masters. These teachers were video recorded in a pan-view arrangement as they taught the same lesson to their corresponding students. Students were randomly selected throughout the lesson and were video recorded with a second camera, so that teacher and student behaviors could be coded relative to a systematic observation tool.

This study has used FSA to compare the behavioral systems of two teachers and their corresponding students in their instructional episodes. A category system has been constructed based on the contextual/organismic setting elements, contextual/environmental setting elements, discrete environmental stimulus elements and specific behavioral elements exhibited in the video recorded teaching episodes and evaluated by FSA (Sharpe \& Hawkins, 1992b).

Prior synonymous behaviors delineated by previously designed systematic observation tools such as WVUTES and SOFIT and the categorical system created in the Sharpe and Hawkins (1992b) study have been used to guide the category creation (Hawkins et al., 1983; McKenzie, 2009). Elements not present in these categorical systems or specifically defined by other previously created behavioral analysis tools or settings mentioned, but are present in the episodes have been categorized to holistically describe all stimulus and behaviors present (Sharpe \& Hawkins, 1992b). Anecdotal recording, bulleting everything the teachers and 
students have done, both visual and auditory, occurring in each teacher's environment regarding the two video recordings has been categorized by both researchers (Cooper, Heron, \& Heward, 2007).

An examination of video recordings multiple times throughout the duration of the lessons has occurred to assure that all elements present were categorized. Each element has a key-word descriptor that described the stimulus and behavioral elements. Key-word descriptors have been used in conjunction with the BEST software to analyze the elements in the instructional episode (Sharpe and Hawkins, 1992b).

Once the category system was constructed, data collection using the Behavioral Evaluation Strategy \& Taxonomy (BEST) software was conducted (Sharpe \& Koperwas, 2010). Videos were examined multiple times analyzing each element independently to assure that the frequency and duration of each element was precise and other elements occurring simultaneously did not interfere with the coding. Based on this, there was a layering effect of elements evaluated in realtime. Elemental totals and sequences of teachers and students stimuli and behavior were coded and analyzed. Elemental summaries and sequences were analyzed and interpreted relative to frequency and duration, rhythm, complexity, coherence, and velocity (Sharpe \& Hawkins, 1992b). In this manner, systematic phenomena of elements occurring in each setting were examined and analyses of relationships were presented and interpreted. Standardized open-ended interviews were used after the teacher's instruction to further delineate the relevance of their ongoing education with respect to their teaching performance.

\section{Limitations}

1. This was the first study analyzing the effects on two teachers' behavioral systems regarding ongoing education in physical education. 
2. Setting may not have been homogeneous, adding to variation of teacher/student interaction.

3. Other limitations to this study may have included volunteer bias, sample selection, and geographical location.

\section{Key Terms}

1. Teaching Effectiveness-teacher behavior which drives student behavior, which in turn results in higher levels of student achievement relative to this instruction. Multiple instructional strategies, task-oriented structures and direct instruction may be related to this term (Rink, 2010). The primary determinant of teaching effectiveness is student learning, which can be assessed primarily through ALT-PE and secondarily through instructionally significant teacher behavior chains (Hawkins, et al., 1983; Parker, 1989).

2. Academic Learning Time in Physical Education (ALT-PE)-the amount of time a student spends engaged in a subject matter motor task with a high degree of success (Parker, 1989).

3. West Virginia University Teaching Evaluation System-systematic behavior evaluation system which codes teacher and student behaviors as real-time events that occur during a lesson (Hawkins et al., 1983).

4. System for Observing Fitness Instruction Time (SOFIT)-simultaneous collection of data on student activity levels, context and teacher behavior in physical education (McKenzie, 2009)

5. Field Systems Analysis-a method of behavioral assessment which describes multiple concurrent factors in real-time (Delprato, 1992; Sharpe \& Hawkins, 1992a). 


\section{Research Questions}

1. Were there differences in the two teachers' behavioral systems in the two instructional episodes with regards to frequency and duration, rhythm, complexity, coherence, and velocity?

2. What did the frequency and probability of elements suggest?

3. What elements showed the highest unconditional probability?

4. What did the two event sequence chains suggest?

5. What did the elemental clusters suggest?

6. What did the three event sequence chains elements suggest?

\section{Defining Variables}

The categorizing variables that were measured were determined by an inductive consensus based upon what had been witnessed in the recorded episodes. Reporting the measures was done separately for the within-field and out-of-field teacher. Qualitative examples of behaviors exhibited in this environment were ones which have been examined in similar studies evaluating teacher and student behavior. Teacher and student behaviors have been analyzed by systematic evaluation tools such as WVUTES and SOFIT (Hawkins et al., 1983; McKenzie, 2009). These variables have been created based on previous environments that may not have been all-inclusive based on the environment in this study. Categories in such behavior analysis systems were used as a guide to create elemental categories in this study. Discrete environmental stimulus elements and behavioral elements witnessed were labeled and operationally defined. Key-word descriptors for both teacher and student behaviors were created based on what had been witnessed in the two recordings. Once these behaviors were categorized, coding of the recordings occurred relative to the system created. Data were presented through elemental 
summaries, two event sequence chains, elemental clusters, and three event sequence chains. Dimensional analysis examining: (a) frequency and duration, (b) rhythm, (c) complexity, (d) coherence, and (e) velocity of elements exhibited was used to determine the differences in the behavioral systems.

Standardized interview questions were given to each teacher at the end of the teaching episodes. These were given to explore these two teacher's experiences in their ongoing education, how they felt it may have influenced their teaching and to supplement the behavioral systems data. Other questions addressed how they felt they would evaluate their teaching before the master's degree and why they received a master's degree within or out-of-field. These were audibly transcribed. 


\section{CHAPTER 2}

\section{Review of Literature}

This literature review has been written to examine the attainment of expertise, effectiveness and the role of experience and ongoing education in regards to six themes: (a) Expert teacher literature, (b) effective teacher literature, (c) gaining expertise through experience literature, (d) ambivalence in gaining expertise through continuing education, (e) measurement of effectiveness and expertise, and (f) field systems analysis.

\section{Expert Teacher Literature}

The term "expert" in early physical education literature has often times been used to describe experienced or effective teaching. Terminology describing expertise usually denotes a list of classifications or characteristics. An example of these characteristics may include: (a) state physical education teacher of the year, (b) recommended by their principal, (c) recommended by university faculty, (d) supervisor/mentor in PE for school district, (e) officer in physical education organization, (f) presented at physical education workshops at the local, regional or state level, as well as a host of other qualifications (Berliner, 1986). As this article suggests, however, these qualifications are oftentimes variable. Furthermore, expertise can rely on teachers' cognitive processes, which even if measured, the processes and actions may differ (Berliner, 1986).

Other authors have cited expertise as teaching effectiveness with a list of similar characteristics that build up to expertise. Dodds (1994) has stated that teaching expertise can include multiple teaching perspectives, dispositions, attitudes, beliefs, knowledge and characteristics that constitute a teacher's makeup. Maximization of student learning amongst other things is another way this expertise definition is defined. Van der Mars et al. (1995) state 
that expertise can be a relative term consisting of many factors including experience, effectiveness, highly skillful teaching exhibition and a host of other factors combined. Sharpe and Hawkins (1992a) suggest that expertise can be generated through a list of characteristics previously mentioned. Behavioral interactions of instructors with certain characteristics can then be measured for differences regarding their behavioral systems. In this manner, a compilation of teacher behaviors in sequence and often times occurring simultaneously can exhibit more expert instruction. These researchers have stated that cause and effect, stimuli and response chains can be analyzed to determine a more expert teaching performance instead of behavioral effectiveness summaries alone. With this synopsis of expertise, a more concrete definition of this term may be ascertained and evaluations can occur.

\section{Effective Teacher Literature}

Regarding teaching disciplines in general, teaching effectiveness has been defined as student learning regarding age and grade level, with a value added to this process equating to more than achievement alone (The Center for Public Education, 2005). Researchers within the field of physical education and coaching have examined the relationship between effective practice and expertise and have determined that effective practice is only one behavioral indicator of expertise (Schempp et al., 2004). Some of the researchers have cited lists of characteristics that maintain and reinforce teaching effectiveness; others cite ALT-PE as the primary assessment variable of effectiveness. Graham and Heimerer's (1981) article on processproduct research resulted in findings relative to teaching effectiveness and identified ten categories that should be examined based on behavioral and classroom climate that promotes student learning. These findings detail the importance of teacher and student behavior that ultimately result in student cognition and physical achievement. These included climate 
variables: (a) warmth, (b) expectancy, (c) task-oriented, and behavioral variables: (a) student choice, (b) structuring, (c) questioning, (d) praise, (e) feedback, (f) class as whole/individual. The author states these categories must be addressed when in conjunction with direct instruction within the classroom. In this manner, effective teachers were labeled as effective if they met varying degrees of these behaviors during their instruction. The effectiveness was categorized as the ability to have automaticity with the managerial tasks as well as the subject matter tasks relevant of content taught. This article stated that effectiveness was relative to flexibility in classroom structure with clear and concise plans of action resulting in student learning and appropriate skill practice. Effective practice among teachers is in "no way determined by one behavior," but a compilation of behaviors, which in turn contributes to student learning (Graham \& Heimerer, 1981, p. 24).

Methodologies that increase student learning in the classroom consist of eight teaching behaviors listed by Brophy (1982), which include: teacher expectations, classroom control, proper support, dynamic teaching, proper curriculum scope and sequence, multiple opportunities for appropriate skill practice, mastery style approach implemented, and recognizing and modifying lessons to address multiple intelligences and grade levels. Effective teachers should be able to take students for face-value and refrain from expressing preconceived biases towards them in regards to their physical abilities and create a learning environment in which they could meet high, realistic goals.

In a study done by Graham, Soares and Harrington (1983) examining teacher effectiveness, effective teachers were ones whose students were engaged in activities for a longer period of time. Effective teachers also exhibited students who spent less time waiting to be engaged in an activity. This study described the effective teacher as one who engages the 
students in high levels of activity while decreasing the amount of time that students are waiting for instruction. This study reinforces Siedentop's viewpoint relative to ALT-PE's barometer role in measuring teacher effectiveness, as well as Metzler's about time on task relating to student learning (Metzler, 1989; Siedentop, Birdwell, \& Metzler, 1979).

Along the same lines of examining effective practices employed by teachers, Brophy (1982) suggested that teachers must effectively follow curriculum by which students are able to successfully meet the designated task criteria by at least eighty percent. He stated that if teachers are able to match task structures towards student's perceived competence in regards to a skill; appropriate lesson planning can be effectively designed. He concluded that having a baseline of student's perceived competence can create a starting point by which more comprehensively designed task structures can be planned and implemented. This article continued with instructional variables that exhibited effective teaching practice. These included lesson planning which targets student outcome variables. Increasing ALT-PE was another a key factor mentioned when determining the proper structure, scope and sequence of a lesson plan. Teaching to a mastery level was also noted by Brophy, in that students should have an opportunity to meet all the requirements of achieving proper skill if the criterion is adequately achieved. When addressing gender and all types of learners within the classroom, a key effective practice stated was that the physical educator requires the same amount of effort from all individuals and does not elevate or neglect certain individuals within the classroom. His concluding statements stated that all individuals in an effective teaching environment should receive equal amounts of instruction and feedback.

In a review article by Harrison (1987), behaviors and strategies for teaching effectiveness were cited. The article addresses the overwhelming question in teaching effectiveness literature 
examining what behaviors categorize some teachers as being more effective implementers of instruction. The article began by stating a caveat in regards to teaching effectiveness delineating that the term can at times be cumbersome due to the environmental complexity. The article stated that one can take the approach of examining ineffective teacher practices as well as the effective to determine differences in the teaching approach. This article on previous literature that examined ineffectiveness provides themes relative to student learning. Ineffective practice detailed less praise, less expectation, both cognitive and skill oriented, less evaluation, less questions answered, less instructional strategies, more criticism and less acceptance to student's ideas and/or student choices. Managing of classes in a manner which increases ALT-PE was delineated in this article as being an effective behavior based on the fact they provide students with adequate chances for skill development. Management and structure alone, this article stated, were not always indicators of best practice, because it sometimes reflected a "busy, happy, good" classroom environment that didn't address student learning, just a custodial approach to teaching (Placek, 1983).

Harrison's (1987) article stated that creating an atmosphere where expectations toward all students should be similar regardless of gender or ability differences should be the goal. Examination of proper behavioral criteria relevant of effective practice in regards to management detailed categories such as with-it-ness, overlapping, flexibility of transition, active participation and student accountability. With-it-ness was defined as a teacher's awareness of what is going on in the classroom at all times, or "having eyes in the back of one's head" (Kounin, 1970). Overlapping was described as a teacher's ability to monitor and implement two separate strategies without decreasing the effectiveness of either strategy. Flexibility of transition details the teacher's ability to efficiently lessen wait time and interim time of students by proper 
instructional strategies. Wait time was described as down time within the lesson in between tasks and interim was defined as the time within a task where a student is not actively engaged in motor appropriate behavior. In this transitional time, teachers who were effective decreased the time in which students were not actively engaged. Active participation details the student as playing a part in the lesson or the sum of all parts, in which all individuals in the classroom should be participating. Student accountability was stated as a proper contingency that created and reinforced behavior relative of skill accomplishment. These criteria detailed behaviors that exhibit effectiveness in regards to management and lesson flow which should lay the groundwork for implementation of lesson content. Other effective management behaviors of effective teachers this article concluded dealt with physical educators being forthright with their students on the first day of class by laying down the rules, routines and procedures that will occur during the course of the year. The article states that providing a student with an initial success at the beginning of the year is essential, so that they feel a sense of accomplishment, as success builds confidence. This article cited examples of the importance of order and instruction and how proper order implemented can increase time on task for the students. If students are offtask, providing positive feedback as well as pinpointing based on proper student behavior can alter this off-task behavior due to the absence of reinforcement it receives. A learning environment that addresses individualized needs, positive atmosphere and opportunities for students to ask pertinent questions was stated to be a part of any well-devised instructional setting.

The literature in dynamic teaching styles discussed the effects of the direct approach style in regards to student learning, and results suggested that higher skilled individuals work well with this approach, but modification of instructional strategies in which students can ask 
questions and have choices has been shown to effectively increase this achievement. Direct instruction was defined as providing the student with a teacher-controlled learning environment with a clear focus on academic goals through structured learning activities, high engagement and feedback (Rink, 2010). This direct style approach was defined as a task-oriented approach and should include outcome goals which should be explicitly stated by the teacher with sequenced and structured organization of what should be accomplished by the student. This teaching strategy is one defined by which effectiveness of practice can be attained when teachers limit student choice, create a classroom environment which is businesslike and they are in total control, and the learning was a result of the teacher providing specific questions and feedback relative of tasks the student had to perform. The indirect teaching style was noted by the article as having characteristics denoting affective learning, in that students have a choice which promotes a creative, student-centered learning environment that may excite certain students into participating. In regards to student learning, the article stated that the direct approach has been found to correlate more effectively with student learning, but the indirect approach is a way of addressing other types of learners who react more positively to this form of instruction. In conclusion, multiple instructional strategies were cited as ways an effective teacher meets the goals of the lesson content and students. Having a repertoire of knowledge to meet the needs of each student was part of Harrison's list of effective techniques.

According to Metzler (1989), the relationship between functional time and student learning outcomes have shown moderate to strong correlations in multiple studies addressing ALT-PE and student achievement. In other words, the student being given more time in purposeful practice correlated positively with student learning. Based on this evidence, effective practices among physical educators, Metzler states, should reflect behaviors conducive of 
instructional feedback, skill statements and instruction relative to student skill practice. The article stated that only about two-thirds of class time even has the potential to be functional time. Of this two-thirds time, minutes allocated to skill learning could only occur about $20-50 \%$ of this time. The article concluded that total time of ALT-PE that could occur was only about $10-20 \%$ of all class time. These are data which related to time and actual student learning outcomes that occurred in one instructional episode.

According to Fink and Siedentop (1989), effective teachers established routines, rules and expectations the first day of school. This was determined based on the fact that management issues could be covered early on in the year and that proper instruction relative to student skill practice would still be available. Their study examined practices of effective teachers with varying years of experience, three with 12 or more years, two with three to five years, and two first year teachers, and examined the routines, rules and expectations these teachers had for their students. The most effective routine exhibited was the attention-quiet routine in which a command was given to gain students attention. The article suggested that positive verbal feedback was the teacher behavior most often exhibited. Specific feedback was exhibited more than general with all of the participants except one, who did not receive training at the same university as the other five in the study. In regards to the rules portion of this study, only one of the teachers out of the six posted the consequences for breaking the rules, and no consequence was ever observed for breaking a rule in any of the teachers' classes. Instead, teachers provided positive feedback towards the students who were complying with the rules, redirected students who were not listening to directions and/or not staying on task, and provided corrective feedback. The expectations teachers had for students were based on process variables not outcome variables, but teacher behaviors didn't reflect these expectations. Effectiveness in this 
study was not attributed to years of experience based on the behavioral similarities exhibited between each of the groups.

Research by Werner and Rink (1989) examined task presentation, nature of feedback and appropriateness of student responses relative to that teacher's behavior. Behaviors by four physical educators in multiple settings and with varying years of experience, but all classified as experienced, were examined by the Qualitative Measures of Teaching Performance Scale (QMTPS). It was determined that teacher's clarity of verbal instruction, knowledge of content, accountability mechanisms, appropriateness and accurateness of cues and ability to focus students were key aspects which determined teacher effectiveness. This study further exemplified the need for effective teachers to promote and exhibit explicit teacher expectations as these were related to creating an environment where students knew their roles. Effectiveness was linked to providing students with the proper amounts of practice trials as well as proper amounts of feedback throughout the lesson regarding skill attempts. This study concluded that exhibiting good management behaviors was not a reliable predictor of student achievement. Effectiveness of instruction reflected by this article's data was exhibited by the quality of skill practice of the student, not only the quantity of time involved in an activity.

A study by Hastie (1994) examined selected teacher behaviors and student ALT-PE in the secondary school setting. It was determined that direct instruction was the instructional strategy employed. Students were given task instructions and teachers monitored students while student behavior was analyzed. The more effective teacher in this study, relevant to ALT-PE, spent more time in concurrent instruction and intervening instruction, while the less effective exhibited high degrees of observing behaviors. Concurrent instruction included teacher feedback comments that were provided to students directly after or during their performance and 
intervening instruction was used to influence future performance. In this way the effective teacher was able to evaluate and analyze student performances. By means of this form of described effectiveness, relevant to ALT-PE increases, effective teachers interjected feedback during and after performances to alter student movements for future effectiveness. The teacher who gave the most information about the task reflected the greatest amounts of ALT-PE from their students.

Research by Walls, Nardi, von Minden, and Hoffman (2002) examined 90 prospective, novice and experienced teachers' comments based on what an effective teacher reflected in their instruction. It was determined through phrases these teacher's surmised that effective teachers had attributes which were enthusiastic, aware, provided students with new concepts, encouraged questions and created an atmosphere in which students were relaxed, and these teachers felt responsible for their student's learning.

Based on Rink's (2010) book Teaching Physical Education for Learning, effective teachers use a multitude of instructional strategies including peer teaching, task-oriented structures and direct instruction. In this manner, effectiveness could be ascertained through a multitude of teaching techniques which addressed student learning. Instructional strategies that were found to be effective for the primary grades included students having their own pieces of equipment, short durations of activities due to attention span, student success through multiple ways to address a task, and creative play. Strategies which were found to be effective in lower intermediate grades included giving cues one at a time, providing feedback which was specific and congruent to the task, multiple positioning of students or use of different equipment in the classroom, and providing the proper amount of refinement cues to increase skill acquisition. In an article by James and Cruz (2005) that recommended strategies for teaching upper intermediate 
grades, increased student independence, including teamwork approaches, cues for evaluation that could be accomplished by self and peers, and students being cognizant about their completion of skill were outlined. These previous strategies outlined were specific to effective practice within the elementary and secondary settings based on age-appropriate and developmentally appropriate activities.

Research by Ha, Chan, and $\mathrm{Xu}$ (2002) examined teacher behaviors as well as student motor appropriate behavior in the junior high setting. Process-product behaviors were collected and it was determined that Shanghai physical educators were more effective in the classroom than their Hong Kong counterparts by students' percentages equating to $40.1 \%$ and $22.4 \%$ respectively in regards to ALT-PE. This study detailed that the Shanghai physical educators incorporated integrated skill-drill and fitness together, while the Hong Kong physical educators used a traditional skill-drill and fitness model that were separate in design. When asked why they used this infused method, they stated it was done to efficiently address the time constraints. The Hong Kong educators spent more time in pre-instruction and management, which this study detailed as the less effective teaching behaviors. This study delineated the relationship between teacher behaviors causing the change in student behaviors. If the Hong Kong educators were able to decrease pre-instruction and management behavior and integrate skill-drill and fitness together, they may have been able to increase the opportunity for motor appropriate behaviors in their students, in-turn increasing their effectiveness.

In an article by Beighle and Pangrazi (2002) entitled Seven Habits of Highly Effective Physical Education Teachers, effectiveness of instruction was attempted by listing habits of effective instructors in the physical education field. This article was an extension of the Steven Covey book (1989) entitled, The 7 Habits of Highly Effective People, as it represented what 
effective practice constituted, and strategies to implement these procedures within the teaching environment. The authors stated that effective and often times experienced teachers have been found to be effective planners and want to know everything they can possibly can in regards to the learning environment in which they will be engaged. Systematic rules, routines and procedures should be set into place at the beginning of the year, so that proper protocols are known by the students. Creating a visual rule list with pictures that represent each rule should be designed and placed in a location in which all students can have proper reference. In regards to rules, the article stated that effective teachers should not only provide consequences for rules, but also reinforcement of these rules for proper contingencies to reinforce or divert behavior of students.

The article stated that effective teachers never should use physical activity as punishment or remove physical activity, if individuals are not behaving appropriately. Reinforcing positive behavior is a habit that this article exemplified, as it provided an affirmation of proper behavior in regards to students who should be listening properly and creates a reference point by which other students may try to attain, due to the praise they will receive. This praise of proper behavior in the classroom was an effective strategy described that stated could create a situation where inappropriate behavior could be curbed due to a lack of reinforcement to the negative behavior and positive reinforcement relative of what has been deemed appropriate. Effective practice was described by the article as one that addressed student goals which has been categorized as motor appropriate behavior. The article continued further by stating that setting high standards which students can achieve will keep students engaged and excited about the skill they are performing. Setting detailed criterion was also described, so that students can adequately achieve performance levels in a manner that addresses task oriented structures more efficiently to 
address student's individualized needs. Modifying task structures and individualized student needs was determined to keep students on task, as it addressed all levels of student skill development. Maintaining general and specific observation throughout the classroom, and being able to efficiently scan the environment for possible issues and concerns along with moving throughout the environment while keeping a centralized location for students, were cited as effective practices. Development of effective lessons was another area of importance in regards to effective practice. Flexible lesson planning which address specific objectives relative to teaching style, multiple intelligences, scope and sequence was another habit mentioned in this article. Proper reflection about lessons taught relative to what happened during the lesson, which reflected effective teaching practice(s) along with what did not happen and what should have happened was detailed as effective practices to create more efficient instructors in the future. Elsewhere, Pangrazi (2001) stated that "peer observation, as well as videotaped analysis with systematic observation and feedback are essential techniques to increase efficiency of practice" (p. 9).

In a study by Zeng, Leung, Liu and Hipscher (2009) effective teaching was represented by teachers exhibiting a multitude of characteristics. These characteristics included: (a) clarity of objectives and content covered in the lesson, (b) proper scope and sequence of expectations relative to student performance, (c) providing proper allocated time for student engagement and success, (d) smooth and quick transitions between activities, and (e) small amounts of management behaviors.

In an article by Webster (2010), teachers used effective communication skills which increased motivation among their students. As prior research has delineated, this article addressed effective practice relative of communication skills that the teachers have exhibited. 
Some of these practices stated here included multiple instructional strategies, clarity of process and purpose, feedback and listening skills, and the use within the instructional setting. The article stated that if students are cognizant to the belief they are gaining positive attributes relative to the skill being practiced, and that teachers have their best interests in mind, motivational levels may increase.

A multitude of qualitative characteristics, lists, criteria and strategies have been discussed as a means of describing teaching effectiveness. As well, articles have cited ALT-PE or student learning as the primary determinant in assessment of teacher effectiveness. The ultimate gauge of effective practice has been determined as a compellation of teacher behaviors which drive student achievement. Or in other words the product of one's instruction, which encompasses the term: student learning or ALT-PE. On that note, the consensus of many of the experts in the physical education field has stated that the most effective way to determine if a teacher is effective is to examine teacher's instructionally significant behavior and student learning, not a solitary teaching behavior (Hawkins, et al., 1983; Sharpe \& Hawkins, 1992b; Sharpe et al., 1989; Silverman, 1991). Other variables can play a part in qualitatively describing teacher effectiveness, but as previously stated, in the physical education environment, student achievement has been the key determining factor in measuring teacher effectiveness. The means of evaluating teaching effectiveness within the physical education field has been accomplished by evaluating these student process variables or behaviors, which leads to a product of instruction. The characteristics, lists, criteria and strategies can be measured, but are not directly related to student outcome (Rink, 2010). 


\section{Gaining Expertise Through Experience Literature}

Research has suggested that expertise can be gained through years of experience. Lists of characteristics along with effectiveness have described the more expert instructor (Griffey \& Housner, 1991; Housner \& Griffey, 1985; Siedentop \& Eldar, 1989; Vogler et al., 1992; Zeng, Leung, and Hipscher (2010). Behavioral systems which have shown to reflect more expert practice regarding years of experience are also cited (Sharpe \& Hawkins, 1992b).

In a study done by Housner and Griffey (1985) on experienced teachers versus inexperienced teachers, experienced teachers detailed more inquiry about environmental contexts before the lesson was taught. During the lesson, experienced teachers detailed almost two times as many instructional strategies as their inexperienced counterparts. Experienced teachers were identified as having five or more years of service in their field. The researchers found that experienced teachers were more attentive to student performance and individualized attention, while inexperienced teachers were more concerned with the class as a whole. The results of this study exposed themes in regards to experience in the classroom. The student learning priority of the experienced teacher was evident in this study as opposed to the custodial, managerial, survival approach of the inexperienced teacher. Examination of this study provides valuable insight into what behaviors experienced teacher's exhibit relative to the physical education environment. This research suggested that experienced teachers were more apt to ask questions of their students and to provide specific observation and feedback relative to the students' performance. In contrast, the inexperienced teachers provided general observation and feedback with a lack of general depth of focus. It was determined that student performance was the primary cue that caused the experienced teacher to adapt their lesson, but the inexperienced made changes in their lesson based on student interest. Clear evidence of differences in 
behaviors and schemata were exhibited between the experienced and inexperienced teacher in this study.

Furthermore, the Housner and Griffey study (1985) also examined the way in which experienced teachers focused their planning and implementation on individual student achievement, while the inexperienced focused their attention on the general interest level of the students. These experienced teachers were able to address not only the managerial aspect within the class, but also achieve assessment and feedback relative to the students. These teachers wanted to know not only where they were teaching, but also the equipment they would be using while they were involved in the study. The experienced teachers were noted as instructors who provided students with a vast amount of information, much greater than that of the inexperienced instructor.

Siedentop and Eldar (1989) reviewed the relationship between expertise, experience and effectiveness, and they concluded that expertise occurred within only certain activities and contexts. The article determined that experienced teachers and often labeled expert teachers were able to have lessons flow appropriately within the time allotted, reinforce certain behaviors intentionally, and allow for flexibility in their lessons while addressing skill related content. In this examination, experienced teachers were able to formulate a plan which addressed the implementation of goals from the beginning of the year, as students were placed in this mode of action and began skill practice more effectively and quickly than the inexperienced teachers. Experienced teachers' students had a lack of confusion and had knowledge of their roles and routines. So, not only were the teachers more efficient in reinforcing behaviors relative to proper motor skill acquisition, but they were cognizant in classroom design and implementation of structure. Automaticity of proper behavior was exhibited by these experienced teachers. These 
experienced teachers with expert characteristics that were shown to be effective did not exhibit managerial procedures that emulated the busy, happy, good philosophy of the inexperienced, which have been implicated in many of the physical education classrooms across the country (Placek, 1983). These experienced teachers also reflected appropriate amounts of student engagement in the activities taught within the lesson context. In conclusion, this study evaluated the thought processes of experienced versus inexperienced teachers and found significant differences.

Similar research on experienced teachers with expert characteristics was completed by a Griffey and Housner (1991) study. These researchers examined not only the thought processes of the teachers, but also the teacher and student behaviors within the classroom. Experienced versus inexperienced teacher's planning was examined in this study and how it related to student behavioral changes. In other words, this was a process-product study determining student behavior change relative to teacher planning. In this study, experienced teachers had five or more years of experience and were "known to be effective and respected" by other teachers and the principals in their schools (p. 197). The experienced teacher was cited by giving much higher amounts of information-giving in their classrooms. It was concluded that experienced teachers commanded more attention of their students with direct style teaching that allotted time for student discussion and comment. In the experienced teacher's classrooms, the experienced teachers were able to maintain control during the lesson and student cognition and openness toward discussion was eminent. Experienced teachers in this study maintained a classroom demeanor which maintained order by addressing individual student needs as well as being able to scan the classroom as a whole if problems occurred. The instructional strategies and planning of these experienced teachers kept order indirectly, while exhibiting the proper managerial behavior 
and addressing student's needs relative to their skill practice (Griffey \& Housner, 1991). Management, assessing student activity and feedback were the primary differences shown by these experienced teachers compared to the inexperienced teachers. Results suggested that students in the experienced teacher's gymnasium were more optimistic relative to the teacher's instruction. The experienced teachers in this setting were able to make decisions more quickly, and when issues arose that deviated from their lesson planning they could adapt with much more ease. These experienced teacher's behaviors reflected less student wait time. However, it was determined that student on-task time was no different in the experienced teacher's classroom when compared to that of the inexperienced.

A similar study examining the role experience plays in teacher behavior was done by Sharpe and Hawkins (1992b) involving case study data which examined the role of an experienced teacher with expert characteristics. The expert teacher had not only experience, but was also a "teacher of the year" award winner. This teacher also exhibited behavioral systems that were consistent with effectiveness within the teaching environment. In this study, a comparative analysis was done examining the behaviors of this experienced teacher versus a novice. It was determined that the behaviors of the experienced teacher reflected high instances of encouragement, verbal instruction prompts, skill statements, and instructional feedback. The novice, on the other hand, exhibited more indecision, contradiction, and an overall management approach within the classroom. The results also suggest that the experienced teacher's ability to exhibit centralized positioning within the classroom along with high degrees of modeling and physical guidance is greater than that of the less experienced teacher. The experienced teacher exhibited a higher rate of change of behavior, and also made these behaviors coherent, consistent and probable, relative to each other. In other words, if one behavior was exhibited, such as 
encouragement, one could predict a behavior such as positive feedback and so on. This behavior chaining that constitutes instructional relevance and higher order thought processes is consistent with the experienced teacher's cognition relative to student needs. This experienced teacher was able to address the classroom as a whole as well as teach individualized content with feedback to specific individuals. Based on this study's results this experienced teacher was more effective based on their instructionally significant behavioral chains. Their behavioral characteristics along with their long list of attributes reinforced the title of having expertise in the field.

This study by Sharpe and Hawkins (1992b) examined an individual who had years of experience, but was also efficient in classroom mobility. This was witnessed by their degree of movement around the classroom and involvement with each student. This expert teacher was shown to have a classroom presence which was comprised of enthusiasm and importance placed on student learning. Examining these experienced teachers with expert qualities, as prior literature has stated, can differentiate quality instruction from mediocre; such teachers develop and revise physical education curriculum, and develop expertise standards relative to "best practice" within the field (Berliner, 1986). This study concluded that future research examining student behaviors relative to teachers' could provide a richer description about how student behavior is reflected in an expert teacher's instruction.

Other literature examining expertise, effectiveness and experience have examined student learning as the criteria for teacher assessment. Measurement of ALT-PE is an efficient evaluator of how much time on task the student exhibits with a high degree of success, relevant to motor appropriate behavior. Motor appropriate behavior is defined as a subject matter motor activity a student engages in with a high degree of success within the physical education environment (Hawkins et al., 1983; Parker, 1989). ALT-PE is an acronym for academic learning time in the 
physical education setting, which can be measured through systematic observation. Teacher and student behaviors can be coded and systematically observed and evaluated by use of a multitude of measurement tools (Siedentop, Tousignant, \& Parker, 1982). Evaluation tools which determine teaching effectiveness through ALT-PE indirectly measures student achievement. Research that has examined the effectiveness of the expert, experienced and novice teachers has found mixed results in regard to student motor appropriate behavior. In a study by (Vogler et al., 1992) examining the role years of experience plays on student motor appropriate behavior, there was found to be no difference when examining behaviors exhibited by inexperienced versus experienced teachers. Experienced teachers in this study were categorized as having six or more years of teaching experience. The experts had to follow the experienced teacher criteria as well along with having four or more of the categories of the following: (a) state physical education teacher of the year, (b) recommended by their principal, (c) recommended by university faculty, (d) supervisor/mentor in PE for school district, (e) officer in physical education organization, (f) presented at physical education workshops at the local, regional or state level (Berliner, 1986).

Within this study, it was determined that expert teachers were more technique focused and the experienced were more fitness focused, but results showed no significant differences in student motor appropriate behavior between the novice, experienced and expert instructors. It was suggested that all other subcategory behavioral differences detailed negligible differences between each group. It also concluded that expertise was once again relative to the context and content, and that experienced teachers in this instance exhibited similar behavior as the individuals labeled as expert and novice. All teachers in this study had behavioral similarities in 
their planning and instruction that were not only efficient, but flexible in their instruction throughout the teaching experience.

Similarly, research by van der Mars et al. (1995) examined novice, experienced and expert teacher and student behavior. The novice had taught two years and under and the experienced only had three or four years of teaching time. The expert teachers were individuals with years of experience and had three or more of the characteristics in the previous Vogler et al. (1992) study: (a) state physical education teacher of the year, (b) recommended by their principal, (c) recommended by university faculty, (d) supervisor/mentor in PE for school district, (e) officer in physical education organization, (f) presented at physical education workshops at the local, regional or state level. In this more recent study, the experienced and expert teachers had lower interim and wait time than their novice counterparts. These results suggested that the organization of these experienced teachers and experts reflected less of time in between tasks than that of the novice teacher's instruction. The experienced and expert teachers were able to maintain attention of their students through proper planning of activities, and did not go through the motions in the classroom from a managerial or custodial standpoint. However, an interesting finding of this study suggested that students, under the instruction of the experienced or expert teacher, exhibited no statistically significantly difference in student motor appropriate behavior.

Research done by Al-Mulla (2002) examined the ALT-PE behaviors of experienced versus novice elementary school teachers in Bahrain. It was determined that in the subject matter motor category no significant difference existed between groups; however, the novice teachers allotted more time for fitness behaviors and game play. This study also concluded that motor appropriate behavior was exhibited by students within the novices' environment in a higher frequency and showed a statistically significant difference from that of the students within the 
experienced teachers' environment. These results are interesting, as it mirrored the previous study by van der Mars et al. (1995) revealing the novice's instruction reflected student motor appropriate levels greater than that of the experienced. Although the van der Mars results were not significantly different, the motor appropriate behavior of the students from the experienced teacher was less than the novice teachers'.

In a study done by Zeng et al. (2010), college instructors, experienced physical educators and inexperienced student teachers were compared in terms of teaching behaviors to determine if there was a difference in effectiveness within their instruction. College instructors and experienced teachers all had to have five years of experience to be placed in this category and the inexperienced teachers were student teachers in the field. It was determined that the student teachers had significant differences from both the other two groups in wait time, controlling, and informing behaviors. College instructors had significantly higher percentages than the other two groups in structuring and cognitive engagement and provided more question and feedback than the other two groups. The experienced teachers detailed the highest percentage in motor engagement at $56 \%$ of class time which was significantly different than the other two groups. However, on a positive note the college professors and student teacher's percentages were still high in this category at $47 \%$ and $43 \%$ respectively. This study's percentages for motor appropriate behavior time were higher than those represented by studies previously citing $20 \%$ as the upper norm (Metzler, 1989). Examining these activity levels, the experienced teachers were over the level of the $50 \%$ recommendation for moderate to vigorous physical activity (MVPA) and the other two were approaching the recommendation percentage needed to meet the Healthy People 2010 standards for activity levels in the physical education environment (US Department of Health and Human Services, 2001). Based on the examination into the experienced teacher's 
literature, one must ascertain, based on the research, that years of experience may be one way expertise can be developed within the physical education environment.

\section{Ambivalence in Gaining Expertise Through Continuing Education}

Experience may be one way to gain expertise; however, another way expertise may be gained is thorough ongoing education. In the U.S Department of Education's (2011) state by state evaluation of certification requirements, more and more states are requiring master's degrees to receive permanent certification as a public school teacher. Often times this legislation has been the driving factor for teachers to complete this master's degree coursework as well as financial incentive. However, the master's degree that one must obtain does not necessarily have to be within one's discipline and can even be completed online. Research has suggested that individuals obtaining master's degrees which are educationally based, and not discipline specific have increased to upwards of $90 \%$ (Terry, 2009). The question then still remains, does obtaining ongoing education in any manner result in an expertise gain? Due to the lack of information regarding the role a master's degree plays in gaining expertise within physical education environment, we must examine the literature in the other educational disciplines.

Master's degree out-of-field literature. Other fields have examined teaching effectiveness and ongoing education. Teaching effectiveness, defined as constituting only one behavioral component of expertise, has been the only evaluative variable in regards to ongoing education to date. Based on this evidence, research on gaining effectiveness relative to ongoing education will be reviewed. Research examining teacher effectiveness in regards to obtaining a master's degree out-of-field has suggested that little, no and even negative relationships exists between attaining a master's degree and increasing one's teaching effectiveness (Campbell \& Lopez, 2008; Clotfelder et al., 2007; Harris \& Sass, 2007; Rivkin et al., 2005). 
A study conducted by Rivkin et al., (2005) which evaluated the effects of obtaining a master's degree in general on teaching effectiveness resulted in no effect on student achievement. In this analysis, longitudinal data including over 200,000 elementary and middle school students were evaluated to determine if a relationship existed between teachers' education and student achievement. This assessment was accomplished by analyzing students' achievement in math and reading before and after instruction. A value-added model was used to analyze data by including students' prior achievement. A value-added model of analysis controls for variables such as teacher and school characteristics. By this type of evaluation, the degree of influence each teacher had on the achievement gain was effectively obtained, while controlling for other variables.

Clotfelter et al., (2007) examination of teacher credentials and student achievement unearthed disturbing results in regards to non-content specific advanced degrees. Individuals in this study were labeled as master's degree receiving teachers that received advanced degrees to obtain higher pay. This study evaluated longitudinal data from $3^{\text {rd }}, 4^{\text {th }}$ and 5 th grade students in North Carolina. In this study, it was determined that a master's degree in general did not result in any statistically significant increases in student achievement in regards to reading or math. In some cases, there was a negative coefficient in regards to an advanced degree and student achievement. Once again, these students were evaluated in a manner which included their previous achievement to efficiently assess the teachers' role in student achievement relative to the teacher's instruction.

In a study by Harris and Sass (2007) examining Teacher Training, Teacher Quality and Student Achievement, researchers discovered that in the elementary setting there is no correlation between a master's degree and student achievement. Furthermore, it was determined that 
obtaining an advanced degree resulted in a negative effect in student achievement in middle school reading and high school reading and math. There were minimal gains in middle school math in regards to an out-of-field advanced degree, but these were not significant. Evidence from this study suggested as well that positive teacher productivity was related to content oriented advanced training and that negative productivity was related to pedagogical advanced coursework. Other interesting findings of this study concluded that content oriented professional development also correlated positively with student achievement in the middle school and high school settings.

Another study by Campbell and Lopez (2008) evaluated the relationship between advanced degrees and student performance on the Georgia High School Graduation Test. Results suggested that even though monetary incentives were used to persuade teachers to obtain an advanced degree, student achievement when in the class of the more highly educated teacher failed to occur. This study controlled for student population, population density, area income, and race. In this study master's degrees in education were cited as the degrees that made the most sense as individuals attained more knowledge and received compensation likewise. Conclusions from this article state the ineptitude and utter fallacy of believing an advanced degree in general constitutes a greater degree of teaching effectiveness. This study dictated the importance of student achievement being the primary factor that should be tied to incentives, not advanced degree attainment. Continuing in this vein, monetary incentives were cited as reasons these teachers continued their education; student achievement was not the driving factor.

Terry's (2009) research evaluating how poor teacher quality has affected students while simultaneously placed the burden on taxpayers for these advanced degrees details the lack of purpose for the out-of-field master's degree. This article detailed how having a master's degree 
did not equate to enhanced instruction or correlate with higher teacher effectiveness relative to student achievement. It states that the "one-size-fits-all" ideology has done nothing except increase cost for school districts who pay for these degrees, or who pay increased salaries for those who acquire them. It continues with stating that most master's degrees that teachers obtain are educationally based, which focus on educational administration and not their content area or best teaching practices. The article states that upwards of $90 \%$ of teacher's master's degrees are completed by means of other educational master's degrees (M. Ed's) and are not in the subject area in which they teach. Most schools give incentives to obtain any master's degree, so the convertibility master's degree, one by which teachers receive pay and also even another focus, i.e. administration, was determined to be the degree which was most often sought out. This trend has shown to increase with the out-of-field master's degree, based on the highest growth rate of all master's degrees attained between 1997 and 2007. This article has reinforced the problem with individuals obtaining master's degrees out-of-field which has been shown to have no effect on teacher effectiveness based on student achievement.

Master's degree within-field literature. Research examining teacher effectiveness in regards to a master's degree within-field has shown much more promising results. In fact, in a study by Goldhaber and Brewer (1998) it was determined that holding advanced degrees withinfield were the only ones that exhibited student achievement gains when compared to teachers holding only a bachelor's degree in the same field. For example, in both the math and science fields it was determined that student achievement increased in the teacher's classroom who held a master's in their respective content. Results were the most significant in math. Furthermore, there was no evidence that obtaining a master's degree out-of-field increased teacher effectiveness. In fact, it was determined by student achievement decreases and graphical 
evidence that math scores actually dropped when taught by a teacher with an out-of-field advanced degree. In conclusion, the data suggested that only receiving a master's degree that was field specific had shown positive results in student learning.

Similar findings by Goldhaber and Brewer (2000) which examined the effects of a master's degree within-field on student achievement concluded much of the same. In an examination of teacher credentials, level of education and type of advanced degree, these researchers analyzed 3,786 math and 2,524 science students and their respective teachers to gain insight into scores relative of these previously described variables. It was determined that students of teachers who held advanced degrees in mathematics scored higher on standardized test scores than any other independent variable defined. Furthermore, in both math and science, obtaining a master's degree within-field was the only independent variable that caused increases in student scores. In science, obtaining an undergraduate degree alone in science did not cause student achievement increases, nor having a master's degree out-of field. However, if a teacher obtained a master's degree in science there was an increase in student scores if the master's degree was within-field. The variable of having a bachelor's degree in mathematics alone resulted in increases in student achievement, but not to the effect of having a bachelor's and master's degree in this field, effect sizes of .41 and .58 respectively. These results were the most evident in mathematics with increases of slightly less than one point on the $12^{\text {th }}$ grade standardized mathematics test.

In a report by The Heritage Center for Data Analysis by Johnson (2000) which examined the effects of teacher training in education on student achievement, it was determined withinfield advanced degrees were substantial, out-of-field degrees were not. The report analyzed student test score data, which suggested that teachers who held advanced degrees in their subject 
increased student achievement. Data from student's National Assessment of Educational Progress (NAEP) test scores in elementary and middle school reading and math were used to validate whether advanced degrees had any value. The control for the effects of socioeconomic characteristics, parents' education and reading material available at home were included in the statistical model. Variables which affected student achievement gains were controlled for as much as possible; however, the instructional methodologies may have varied. The study determined that statistically significant differences in reading and math scores of eighth grade students were evident if their teacher held a master's degree within-field. If a teacher held a bachelor's and master's degree in reading, NAEP reading scores of students of these teachers averaged 2.7 percent higher than those who held other educational master's degrees. If a teacher held a bachelor's and master's degree in math or science, students in these teacher's classrooms averaged 3.4 percent higher scores when compared to individuals with master's degrees in other educational disciplines (M.Ed's). Evidence of importance on specificity of advanced degree was shown to be more evident as the grade level increased. In the elementary setting, scores of students were not significantly different relative to increases in reading or math if an individual held an advanced degree in either reading or math as compared to those who had other educational master's degrees (M.Ed's). The article stated that the value of obtaining an advanced degree within-subject adds additional value in the upper grades as the material is more "rigorous" in nature (p. 7).

Analysis of student data by Betts et al. (2003) revealed that students whose teachers held masters and doctoral degrees resulted in increases in student reading achievement. These data were collected from students in the San Diego Unified School District between the fall of 1997 and the spring of 2000. These students who exhibited achievement gains were both English and 
Spanish speaking. The data revealed that if teachers of students held a Ph.D., students' percentage change in the rate of learning tripled from a master's degree alone. Data revealed that this was not the case for all students in reading or math at the elementary level or in math at the secondary level. Results suggested that subject matter knowledge is more applicable as the student's grade level increases, which mirrors the Johnson (2000) study's math results.

An article by Goe and Stickler (2008) presented similar results when it examined multiple studies regarding an advanced degree's significance. Only degrees that were withinfield were cited as those that increased student achievement. In this brief from the National Comprehensive Center for Teacher Quality, the fields of math and science were cited as fields that had significant changes in student achievement regarding within-field master's degrees of teachers. In the math discipline, several studies concluded the significance of these field specific degrees in increasing student test scores (Aaronson, Barrow, \& Sanders, 2003; Frome, Lasater, \& Cooney, 2005; Goldhaber \& Brewer, 2000). In the science field, discipline-specific professional development, i.e. training in how to set up labs effectively, etc., was shown to increase their students' grade level by as much as $40 \%$. Even though in the science field this is not a master's degree within-field, increases in student achievement were relevant to subject matter professional development, which showed content specific ongoing training positively affecting student learning (Monk, 1994; Wenglinsky, 2000).

Results from the Roza and Miller (2009) article entitled the Separation of Degrees: Stateby-State Analysis of Teacher Compensation for Master's Degrees, suggested that master's degrees within-field are the only advanced degrees that should be rewarded with a master's pay bump. In this analysis, it was determined as well that master's degrees in other educational disciplines (M.Ed's) bear no evidence of increasing teaching effectiveness. According to this 
analysis of data from previous studies evaluating a master's degree's worth, math and science master's degrees have been linked to student achievement gains. This article reveals an interesting trend of gaining a master's degree in education, which was revealed by the highest growth rate of all master's degree programs between 1997 and 2007. Interestingly enough, states like New York require individuals to obtain a master's degree to receive the highest level of licensure. Seventy-eight percent of teachers in the New York state hold master's degrees, however, once again the vast majority are in education, which has shown little or no correlation with increases in teaching effectiveness.

\section{Measurement of Effectiveness and Expertise}

In other fields, research has suggested that discipline-specific master's degrees have played a role in teacher effectiveness gains, which we know is one behavioral component leading to expertise. Within other fields and physical education, however, measuring expertise relative of ongoing education, has been absent. Possible reasons for this neglect may be attributed to the lack of sensitivity in measuring expertise variables including behavioral interactions and the more easily attained teaching effectiveness variables (Hawkins, 1992).

Effectiveness evaluation tools. Effectiveness based on student achievement, or behavioral totals have been analyzed in the physical education environment. In the physical education environment, ALT-PE, which is evaluated by motor appropriate behavior at West Virginia University through WVUTES, is the most salient criteria for measurement of teaching effectiveness (Hawkins et al., 1983; Parker, 1989). Many other criteria can be assessed, but student learning has become the determining factor in teaching effectiveness in the physical education environment as well as other disciplines, and ALT is the most important proxy for student learning. Instructionally significant behaviors of teachers can be measured as well to 
determine if behavioral sequences represent something that is substantially, purposefully orchestrated. Instructionally significant teacher behaviors in sequence, which constitute effectiveness can be assessed by WVUTES along with other categories of student behavior that are proxies for student learning (ALT-PE) (Hawkins et al., 1983).

SOFIT has been used as another method to measure teacher effectiveness. This tool measures moderate to vigorous physical activity (MVPA) levels in students in the physical education environment (McKenzie, 2009). Each of these tools, along with others that measure frequency and duration of lineally occurring elements relative of ALT-PE, has been used to address the teaching effectiveness assessment. Analyzing these behaviors by a systematically derived assessment tool has been able to define differences in effective and ineffective teachers (Sharpe \& Hawkins, 1992a).

Expertise evaluation tool ambivalence. In regards to the analysis of teaching expertise, along with detailing lists of factors that may lead to expertise, complex and subtle factors which may lead to this distinction have been far less examined (Dawe, 1984; Eisner, 1983; Gage, 1978; Rubin, 1985; Sharpe \& Hawkins, 1992a).

One reason for the lack of focus, or evaluation tool absence, in evaluating expertise may relate to the systemic nature of the assessments itself. Behavioral interactions of teachers have been evaluated by effectiveness totals, etc., but not to the degree that addresses the interactions of behavioral systems relating to a specific instructor. In fact, identifying, defining and measuring variables that are not a part of a previously designed systematic assessment tool for effectiveness is a much more arduous process. Once the categorical system is created, it must encompass everything occurring within that specific environment (Sharpe \& Hawkins, 1992a). The process of creating and analyzing an all-encompassing stimuli and behavioral system is 
more time intensive than using previously created teaching effectiveness tools. Examining the interactions of behaviors may provide a richer description of factors that contribute to expert practice. Specifically, an analysis of the temporal locus of behavior, the temporal extent of the behavior, the context where the behavior occurs, and the multiple behaviors which may occur at the same time may address these sensitive factors which may address expertise in teaching.

Factors that may be attributed to differences in an individual's behavioral system regarding that specific field may be related to an individual's teaching experience (Sharpe \& Hawkins, 1992a). In regards to advanced training, previous literature in other disciplines has suggested the same (Betts et al., 2003; Goldhaber \& Brewer, 2000; Roza \& Miller, 2009). Holistic analyses to ascertain all the elements in a teaching episode which exhibit expertise are often times missed in typical cause - effect studies (Delprato, 1992). In regards to this type of holistic field analysis, ongoing education and its significance in gaining expertise in physical education can be evaluated through one's behavioral system.

\section{Field Systems Analysis Literature}

FSA or behavioral systems analysis, analyzing teaching expertise, uses investigative procedures to attain (a) descriptions in terms of (b) multiple concurrent factors (c) operating in real time (Ray \& Delprato, 1989). This method of behavioral systems analysis has taken behavioral analysis to another level, or increases the complexity, by assuring that multiple concurrent factors occurring simultaneously can be properly evaluated (Hawkins, 1992). This method of analysis addresses the complexity of behavioral interactions that may be missed if a categorical system is not created inductively, based on what has been exhibited within this environment. The philosophy of interbehaviorism, which reflects the behaviors being described in field terms, was first described by Kantor (1969). This form of behaviorism included 
behaviors under question relative to the field under examination. This philosophy takes into consideration all the factors in an environment that will provide proper descriptive and explanatory functions relative to multiple factors within that specific environment. Once categorized and created, analysis of variables can occur to assure a rich descriptive synopsis of what has been exhibited. Based on this type of analysis of systems, the cause and effect or stimuli and responses are evaluated along with the interactions of the entire set of factors simultaneously (Delprato, 1992). Interdependent components that occur simultaneously in a system can further explain interactions which may not be discovered by simple linear causeeffect analysis. By means of this type of field systems analysis, interactions then can explain a more expert pedagogue in regards to these interactions regarding dimensions of data analysis.

Previous literature in physical education teaching has stated that more clearly defined teacher behavioral chains and immediacy of such chains in occurrence as well as a combination of teacher behaviors and instructional methods encompass just a few of the variables which describes effectiveness which leads to expertise (Sharpe et al., 1989; Thaxton et al., 1977). In other similar research, McAvoy, Hursh, Nardi, Savage \& Walls (1988) have stated that effective instructors exhibit greater variance of behaviors, greater velocity of behaviors, a lack of criticism, more encouragement, less managerial behavior immediacy reinforcement of behavior and more feedback in regards to the instructional episode. Greater variability in behaviors has also been cited as determining a more effective instructor in other studies (Goldberger, 1984; Graham \& Heimerer, 1981).

In an analysis of an instructor's behavioral system, relating to the multiple behaviors occurring at the same time, or with temporal immediacy, complex interactions that have been previously overlooked can be analyzed. FSA analyzes differences in ones behavioral system in 
regards to frequency and duration, probability, rhythm, complexity, coherence and velocity of the behaviors interacting. In this manner, literature describing these interactions whether in occurring in sequence or simultaneously, one can more efficiently distinguish differences between experts and non-experts (Sharpe \& Hawkins, 1992a).

In the physical education environment, an expert vs. novice study completed by Sharpe and Hawkins (1992a) categorizing behaviors exhibited in this environment and then coding these episodes has been done. In this setting, behavioral stimuli and behaviors that followed were categorized based on the narrative that described what occurred. Key-word descriptors that described the behaviors witnessed were then placed into categories that were of manageable size, based on the computer program alphanumeric availability. Operational definitions for each of the behavioral and setting events were constructed. Environmental and organismic setting elements were the first two parts of the categorical system. These did not change in the duration of the instructional period as they made-up the historical events and characteristics present in the study. Environmental stimulus and specific behavioral components made up the third and fourth parts of the categorical system. Data in this study were then collected based on the instruction of both the expert and novice. Data on the frequency and duration of these environmental stimulus and behaviors were summed. A probability matrix examining a stimulus causing a chaining of succeeding behaviors was created by the computer program. Analysis of this preceding stimulus causing succeeding behavior exhibition was produced in this conditional-probability matrix. A kinematic analysis provided a visual model of how system elements were related, and dual chains with their corresponding frequency and probability of occurrence were listed. Probable elemental sequences were then created to further describe the chain of stimulus-behavior pathways. High frequency elements were nested in a final part of the flow charting. In this 
manner, pre and post-trigger elements were described in regards to the high frequency elements. Graphical representation of these high frequency stimulus and behavior elements, short time-lag interaction of elements, or other interesting kinematic relationships witnessed were plotted relevant to each other and graphed accordingly.

In regards to the dimensional analysis, the expert exhibited a higher frequency and probability of specific dual chains. The rhythm of the expert, regarding the tempo, regularity and complexity of the interactions were more consistent. The expert's behavioral system was more complex, but also more coherent. The expert's system reflected high probabilities of high frequency within fewer dual chains. Finally, the elemental velocity of the expert's system was much larger reflective of more elemental pairs as well as the total frequency of all elemental pairs (Sharpe \& Hawkins, 1992b).

This study cited the need for future studies evaluating student behaviors in relationship to the teachers when examining expert vs. novice instruction. Other process-product studies have examined teacher and student behavior in the physical education setting (Griffey \& Housner, 1991; Silverman, 1991). However, to date, there was no study examining ongoing education in physical education and the role it may play in a teacher's behavioral system. The purpose of this study was to create a categorical system of elements present in these two environments (Sharpe \& Hawkins, 1992b). Once created, the evaluation of the differences in teacher behavioral systems regarding their behavior and their students behavior related to ongoing education was determined. 


\section{CHAPTER 3}

\section{Method}

\section{Participants}

Two $(n=2)$ current physical education teachers with exactly five years of teaching experience in physical education were the subjects in this study. Previous studies have constituted "experience" as teachers having five years of teaching experience and beyond (AlMulla, 2002; Griffey and Housner, 1991).

These two instructors had similar demographic backgrounds and instructional contexts. They were as closely paired as possible, excluding their master's degree education. One instructor was an individual who had obtained an undergraduate degree in physical education and a master's degree in physical education. The second instructor was an individual who had obtained an undergraduate degree in physical education and a master's degree in administration. Both instructors were trained in the same undergraduate physical education teacher education program. The age and years of teaching experience were nearly equivalent in the selection of the participants. The teacher within-field or the one with the master's degree in physical education was 27 years old, while the teacher out-of-field or the one with a master's degree in administration was 28 years old. Participants in this study were both males who taught in the Mid-Atlantic States region at low socioeconomic status schools. The teacher within-field taught physical education in an urban setting, while the teacher out-of-field taught physical education in a rural setting. Individuals selected were both instructors in the elementary setting, teaching soccer to a third grade physical education class. Both individuals in this study have achieved a master's degree within five years. This was done to address the master's degree significance as much as possible, limiting the latency of degree characteristic wash-out effect. 


\section{Research Design}

This was a case study analyzing two teachers teaching one lesson each. Behavioral systems relative to each instructional environment were evaluated. Standardized open-ended interviews of each teacher have been evaluated and have supplemented the behavioral systems data (Patton, 2002). The two teachers involved in this study taught the same lesson content to the same grade level of students. One lesson taught by each teacher was analyzed. The content of the teaching episode, which was soccer, was selected on the basis that both teachers had experience in teaching it and had a high perceived competence regarding the content selection when asked to choose amongst a variety of content choices.

Variables to be measured were determined by an inductive analysis and categorization of behaviors observed in the video recordings (Cooper et al., 2007). Measurements were reported separately for the within-field and out-of-field teacher. An anecdotal observation occurred, recording all descriptive, temporal sequenced accounts of all behaviors relative to antecedents and consequences in each environment (Cooper, 1981). The categorization process replicated the Sharpe and Hawkins (1992b) study's categorical creation.

Contextual/organismic setting elements, contextual/environmental setting elements, discrete environmental stimulus elements and specific behavioral elements observed have been labeled and operationally defined. These key word descriptors for both teacher and student behaviors were created based on what was observed in the two videos. WVUTES behaviors and definitions along with other pedagogically relevant coding systems were used to create these operational definitions (Hawkins et al., 1983). Once these categories were created, each of the teacher and student video recordings were coded relative to the system created. Frequency and duration, rhythm, complexity, coherence and velocity of elemental interactions were used to 
describe the behavioral characteristics of both settings. Each teacher's system was the basis of comparison regarding each teaching episode. Standardized interview questions have added to the context and explanation of the behavioral systems data by providing similarities and differences regarding the two teacher's answers.

\section{Participant Selection}

These two individuals were purposefully selected to meet the criteria for this study. These two individuals selected had to have been teaching physical education for a period of five years or more after they had earned a bachelor's degree in physical education. These two individuals had to have earned a master's degree in physical education or a master's degree in a field other than physical education. One individual who met the previous criteria and who received the master's degree within field was selected. A second individual who met the previous criteria and who received a master's degree out-of-field was also selected. These two individuals had to be of the same gender. These two individuals were purposefully selected from a West Virginia University undergraduate roster database and had to have completed a master's degree in physical education or another field. These two individuals were selected on the basis that they were confident, competent instructors who taught at the same grade level.

\section{Recruitment}

Two individuals were selected if they met the criteria to be a part of this study. Once selected, they were recruited by email and phone to discuss what was to be required of them. The two individuals who were selected for this study went through the West Virginia University's Institutional Review Board (IRB) consent process. Approval was obtained by each of the school's principals to conduct the study and consent forms were signed by each of the teachers in the study. Parents signed consent forms based on their willingness to allow their children to 
participate in the study. Assent forms were also signed by each of these students whose parents allowed them to be in the study (See Appendix F).

These two individuals were told that they would be participating in a study examining teacher behavior and corresponding student behavior relative to advanced education. Once selected, the two teachers were told what content they would be teaching based on consensus reached, relative of their content competence.

\section{Data Collection Procedures}

Participants involved in this study were video recorded teaching the same content for three lessons. Teachers then selected their best lesson and it was analyzed. This best lesson was determined by each instructor. Each teacher made the decision on the instructional episode they felt was most effective out of the three. Video recording of the lessons was of the obtrusive nature, based on the fact students and teachers had the knowledge that the recording was taking place. These lessons were recorded by means of a pan-view arrangement where the instructor's behaviors were viewed throughout the duration of the lessons. The instructor wore a microphone during the lessons, so their statements were audible on the recordings.

A second video recording was focused on students in the context of the instruction. Random selection of students throughout the lessons was done in two minute intervals to achieve an authentic representation of a composite student throughout the lessons' activities. At this point, students were randomly selected for observation for two minutes.

The video recording of both instructors and randomly selected students was done in person as the researcher was on site to set up the two video cameras, video record the lessons and provide the physical educators with the microphone. A second researcher video recorded the students throughout the lessons. 
Verbal field description. A narrative account including the context, participants, stimuli and behaviors in each teacher's best lessons, one each, relative to teacher and student behavior exhibited was produced from the video recorded sessions. Examples of this field description are in the Sharpe and Hawkins (1992b) study.

Categorical-system construction. Based on this field systems analysis, four categories of descriptors were created (Sharpe \& Hawkins, 1992b). Contextual/organismic setting elements, contextual/environmental setting elements, discrete environmental stimulus elements and specific behavioral elements were created based on each setting, teacher, environmental stimulus and behaviors exhibited by the teachers and composite students. Consultation between researchers occurred, so that consensus was reached on what elements were present in the video recorded episodes to ensure an accurate description of what actually occurred (Sharpe \& Hawkins, 1992b). The recordings were viewed multiple times each to assure all elements were targeted effectively for categorization. Attempts to use vocabulary and category definitions that have been used by other pedagogical researchers in this setting occurred to increase the relevance of terminology (Hawkins, et al, 1983; McKenzie, 2009).

After researchers examined the videos multiple times, key-word descriptors were assigned to the stimulus and behavioral elements exhibited (Cooper et al., 2007). Descriptors were then combined and categorized by similarity of function in order to have a resultant allinclusive categorical system that was also manageable. Manageable size was determined by the number of keys on the keyboard which related to the data-collection apparatus (Sharpe \& Hawkins, 1992b). Operational definitions were created for each of the key-word descriptors and a list of definitions for the stimulus and behavioral elements has been provided. Environmental and organismic setting elements which describe both settings and teacher profiles from a current 
and historical ontology are characteristics that do not change through the duration of the teaching episodes. Descriptions of each differed based on the instructors. Environmental stimulus and specific behavioral elements described everything that occurred from the beginning of the teaching episode to the end. An all-inclusive categorical system was attempted (Sharpe \& Hawkins, 1992b).

\section{Data Analysis of Dialogue}

After the categorical system based on descriptors was combined and categorized by similarity and function, a second researcher analyzed the elemental data to assure that the categorical system created reflected what was actually exhibited in the dialogue. This second analyzer randomly selected $10 \%$ of the elemental data to analyze for interobserver agreement. This agreement percentage was obtained by dividing the agreements by the sum of agreements plus disagreements, and then multiplying by 100 . The data analyzed included the teacher withinfield, the teacher out-of-field, the student within-field and the student out-of-field dialogue data. The interobserver agreement was $91 \%$ for the teacher within-field and $87 \%$ for the teacher outof-field. The interobserver agreement was $100 \%$ for the student within-field and $95 \%$ for the student out-of-field.

\section{Instrumentation}

The video recordings were analyzed by means of the Behavioral Evaluation Strategy \& Taxonomy (BEST) software utilizing the categorical system created by the dialogue. The categories of discrete environmental stimulus elements and specific behavioral elements have been used to evaluate what has occurred in each teaching episode. These categories have been created based on a verbal, descriptive chronology of what was exhibited in the teaching episodes. This BEST software utilizes observational real-time data collection (Sharpe \& Koperwas, 2010). 


\section{Data Collection Apparatus}

Each stimulus and behavioral element was assigned an alphanumerical character. (1-9=19, 10=0, 11=A, 12=B, etc.) (Sharpe \& Hawkins, 1992b). Within the BEST software program, start/stop times are represented in terms of microseconds. The BESTVIDEO version of the software was used to synchronize the videos with the data collection process. The start and stop locations of each behavioral element were identified on the video, and the researcher used the keyboard to mark the beginning and the end of each element as the video was run. Multiple runs through the video were used in order to record all the elements in the category system. Errors in recording an element were canceled in some instances and the video was rerun for more accurate data collection.

The recordings have been viewed following the data collection to assure agreement of the researchers regarding the correct categorization of what element was exhibited based on previously defined definitions. These codings were done independently regarding each researcher.

\section{Data Analysis Codings}

After the video recordings were coded using the BEST software in conjunction with the elements in the category system, a second researcher analyzed the codings for interobserver agreement. This second researcher used momentary time sampling to analyze the data for interobserver agreement. For every minute within each the video recordings, data was sampled at a specific time that was randomly selected for interobserver agreement. This agreement percentage was obtained by dividing the agreements by the sum of agreements plus disagreements, and then multiplying by 100 . The data analyzed included the teacher withinfield, the teacher out-of-field, the student within-field and the student out-of-field coded data. 
The interobserver agreement for the coded data was $97 \%$ for the teacher within-field and $95 \%$ for the teacher out-of-field. The interobserver agreement for the coded data was $96 \%$ for the student within-field and $90 \%$ for the student out-of-field.

\section{Data Presentation}

Discrete element synopsis. Stimulus and behavioral element summaries with respect to frequency, duration, mean duration, percent duration and rate per minute have been totaled for the elements. The BEST software has provided these totals as well the frequency and probability of elements that occurred in sequence more often than by random chance. This program has provided these for the high frequency elements.

Two event sequence. Kinematic flow charting was the second part of the data presentation (Ray \& Delprato, 1989). This kinematic analysis provided a visual model of how system elements were sequentially related to each other. Stimulus and behavioral two event sequence chains were presented, relevant to trigger elements that evoked succeeding element occurrence. Frequency of occurrence and Z-scores with statistical significance have been used to guide the chaining process. A two event sequence chain was included if it occurred three or more times in the data and the Z-score was statistically significant at the $.05, .01$ or .001 levels.

From these two data representation formats, probable element sequences depicting a greater portion of the temporal system in action have been represented. Stimulus and behavioral element sequential-field examples or multiple chains in sequence have been visually represented to add to the data presentation. In this manner, primary succeeding elements resulting from a trigger element, along with secondary, tertiary and so on have visually represented the data patterns. Sum totals of types of chains, frequency of chains and probability amongst these chains along with the rate of chains per minute have been summarized. 
Three event sequence. Finally, high-frequency elements that occur as fulcrums in the data within more probable preceding and succeeding element clusters were visually examined. The inclusion criteria for these three event sequence chains was that these high frequency fulcrums had to be ones that occurred more than once in the data in conjunction with the chains that occurred three or more times in the data and were statistically significant at the $.05, .01$ and .001 levels. In this manner, teacher behavior flowing through time was represented in multiple formats explaining the behavioral systems of each instructor. Precursors and successors in conjunction with these fulcrums were then analyzed for differences.

Lag analyses. Lag analyses were also completed to determine the degree of temporal contiguity between one element triggering another in a sequence that was initially hidden by simultaneously occurring elements in a chain. This has been accomplished to determine how soon a chain occurs relative to a trigger element. Differences in the data regarding the lag analyses were summarized.

Graphic representation. Analysis of stimulus and behavioral elements that followed the previous criteria for the two and three event sequence chains have been graphically juxtaposed to present data efficiently. Durations in time have been visually displayed so that slices of the instructional episode have been presented relative to the field system of both teachers involved. In this manner data sets have been able to be graphically represented, so that stimulus and behavioral elements have been charted for duration and frequency. Data patterns have then detailed the overlapping elements throughout the instructional time-frame. The similarities and differences in the data patterns regarding the temporal locus or series of elemental events has been summarized. The graphical representation of elements has been analyzed and conclusions 
have been reached regarding the time of occurrence of elements and the relationships of elements in succession.

This graphical representation has provided a rich description of elements and their interactions in each teacher's instructional episode. Analysis of the graphed data has provided observation and interpretation of elemental events in time.

A synopsis of differences in stimulus and behavioral elements has been compiled for each teacher and their composite student in the teaching episodes. This has been provided in table format to more fully describe what has been exhibited by each teacher and their composite student.

\section{Data Interpretation}

Five dimensions of field systems were used to understand the data exhibited in the systems under analysis. This dimensional analysis was based on Sharpe and Hawkins (1992b) study. These dimensions were focused primarily on the temporal and contextual relationships that were revealed in the data analysis.

1. Frequency and duration: Each individual element was analyzed to determine the number and length of occurrence represent in the systems.

2. Rhythm: This term describes the temporal patterns of elements, chains, or clusters of elements or occurrences of patterns throughout the instructional episode. Frequency and relative probability of temporal patterns were analyzed relative to tempo, regularity and complexity of elements throughout time.

3. Complexity: This variable defines the system based on the number of individual categories that were used to describe the stimulus and behavioral elements in the instructional episodes. This complexity has been determined by the number of categories created. 
4. Coherence: This category described to what degree each of the elements was consistently related to each other. Coherence represents the degree or order and consistency in a setting. The BEST software has determined these probabilities. High probabilities of high frequency within a few dual chains reflect a greater organizational coherence or predictability. Low probabilities of low frequency within many dual chains indicate less coherence.

5. Velocity: The frequency of occurrence for each element and sequence pairs has been analyzed to determine differences in elemental velocity. Differences in elemental velocities regarding both singular and sequence pairs have been summarized.

Each of these dimensions have been used to analyze the data that were presented from the probabilities of two and three event sequence chains, stimulus clusters and complex behavioral sequences. Descriptions based on what has resulted were reflected in these dimensions. Conclusions have been drawn from results relative to each instructional setting.

Proper dissemination of the differences between two behavioral systems with respect to these previously cited dimensions was the primary means of this case study analysis.

\section{Interviews}

Standardized interview questions have been incorporated as a secondary, supplemental means of adding to the context and data explanation of this case study analysis. Questions have been predetermined and both interviewees received the same questions in the same way and in the same order (see Appendix C and D). These interviews have been audibly transcribed.

Data analysis of interviews. A description of what each individual stated in their answers has been compiled. Interview answers have been provided verbatim. Dissemination of how each teacher talks about their teaching and the differences between each teacher's responses and their behavioral systems have been summarized. 


\section{CHAPTER 4}

\section{Results}

\section{Teacher/Subject Contextual Information}

The differences and similarities between the contextual/organismic and contextual/environmental traits for the teacher within-field (T-In) and the teacher out-of-field (TOut) have been described. These elements can be found in Table 1.

Table 1-Contextual/Organismic, Contextual/Environmental Traits

\begin{tabular}{|c|c|c|}
\hline \multicolumn{3}{|l|}{$\underline{\text { Organismic }}$} \\
\hline T-Out & & T-In \\
\hline Gender & Male & Male \\
\hline Age & 28 & 27 \\
\hline Yrs. Taught & 5 & 5 \\
\hline Undergraduate & PETE WVU & PETE WVU \\
\hline Highest Degree & Master's Degree & Master's Degree \\
\hline Type of Degree & Administration M.Ed. & PETE M.S. \\
\hline Degree Obtained & Within 5 years & Within 5 years \\
\hline Other Experience & Coached High School Wrestling 2 years & Coaches Cross Country/Track and Field at High School \\
\hline Athletic Background & Wrestled College & No Collegiate Sports \\
\hline \multicolumn{3}{|l|}{ Environmental } \\
\hline Facility & Half, Full-sized Court & Half, Full-sized Court \\
\hline Equipment & Adequate & Adequate \\
\hline \multirow[t]{5}{*}{ Pupil Traits } & 9 in class & 9 in class \\
\hline & Equal Gender Mix & Equal Gender Mix \\
\hline & $3^{\text {rd }}$ Grade & $3^{\text {rd }}$ Grade \\
\hline & Rural Low-SES & Urban Low-SES \\
\hline & \multicolumn{2}{|c|}{ Similar Activity and instructional history across classes } \\
\hline Instructional Format & 6 stations, groups of 3 & Partners, groups of 2 \\
\hline Skill Content & Soccer (dribbling, shooting) & Soccer (dribbling, shooting) \\
\hline Observational Point & Endpoint of & \\
\hline
\end{tabular}


Before the data were collected, organismic and environmental traits were summarized for each participant in the study. Each individual was male and both were similar in age, at 27 and 28 years of age. Both individuals had been teaching physical education for a period of five years and had received their master's degree within the last five years. Each individual received a different master's degree as the T-In received a master's in physical education and the T-Out received a master's in administration. Each individual had some coaching experience.

From an environmental standpoint, both teachers had adequate facilities and the same half, full-sized court space to teach their physical education lessons. Each teacher in this study taught an equal gender mix of the same number of students coming from the same socioeconomic background, although each coming from a different environmental setting. These students were taught the same content, which was a soccer lesson encompassing dribbling and shooting. Each teacher taught the content with their own type of instructional format. The T-In used pairs of two to teach the content during the instructional portion of the lesson, while the TOut used stations to teach the content. The T-In set up the equipment during the lesson, while the T-Out had already set up the stations beforehand.

\section{Environmental and Behavioral Category System Teacher}

The contextual and behavioral category system that was created for the T-In and the TOut can be found in Table 2 .

Table 2 Teacher Contextual/Behavioral Category System

\begin{tabular}{|cl|}
\hline & Element \\
\hline 1. & General Observation (behavioral element) \\
\hline 2. & Positive Feedback: Specific (behavioral element) \\
\hline 3. & Positive Feedback: General (behavioral element) \\
\hline 4. & Corrective Feedback: Skill (behavioral element) \\
\hline
\end{tabular}




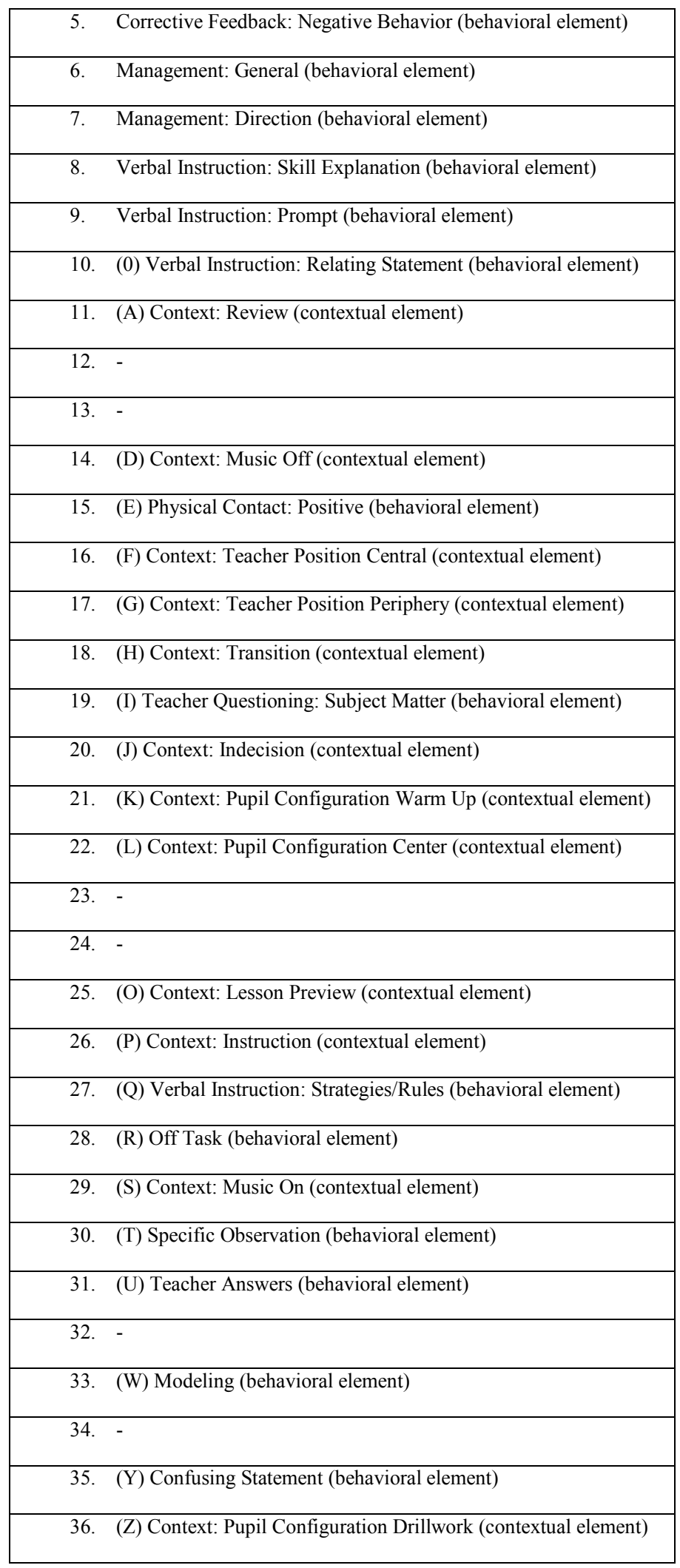


This category system was created after the video recordings were watched multiple times and category systems emerged from the video recordings. This category system was specific to the teacher's environments.

\section{Environmental and Behavioral Category System Student}

The contextual and behavioral category system that was created for the S-In and the S-

Out can be found in Table 3 .

\section{Table 3-Student Contextual/Behavioral Category System}

\begin{tabular}{|c|c|}
\hline & Element \\
\hline 1 & Motor Appropriate (behavioral element) \\
\hline 2 & Motor Inappropriate (behavioral element) \\
\hline 3 & Motor Supporting (behavioral element) \\
\hline 4 & Cognitive: Passive (behavioral element) \\
\hline 5 & Cognitive: Active (behavioral element) \\
\hline 6 & On Task Management: Verbal (behavioral element) \\
\hline 7. & On Task Management: Non-Verbal (behavioral element) \\
\hline 8 & Off Task (behavioral element) \\
\hline & Interim (behavioral element) \\
\hline & (0) Waiting (behavioral element) \\
\hline & (A) Context: Review (contextual element) \\
\hline & \\
\hline & \\
\hline & (D) Context: Music Off (contextual element) \\
\hline & (E) Negative Self-worth Comment (behavioral element) \\
\hline & (F) Context: Teacher Position Central (contextual element) \\
\hline & (G) Context: Teacher Position Periphery (contextual element) \\
\hline & (H) Context: Transition (contextual element) \\
\hline & \\
\hline & (J) Context: Indecision (contextual element) \\
\hline & (K) Context: Pupil Configuration Warm Up (contextual element) \\
\hline & (L) Context: Pupil Configuration Center (contextual element) \\
\hline
\end{tabular}




\begin{tabular}{|ll|}
\hline 23. & - \\
\hline 24. & - \\
\hline 25. & (O) Context: Lesson Preview (contextual element) \\
\hline 26. & (P) Context: Instruction (contextual element) \\
\hline 27. & - \\
\hline 28. & - \\
\hline 29. & (S) Context: Music On (contextual element) \\
\hline 30. & - \\
\hline 31. & - \\
\hline 32. & - \\
\hline 33. & (W) Confusion (behavioral element) \\
\hline 34. & - \\
\hline 35. & - \\
\hline 36. & (Z) Context: Pupil Configuration Drillwork (contextual element) \\
\hline
\end{tabular}

This category system was created after the video recordings were watched multiple times and category systems emerged from the video recordings. This category system was specific to the student's environments.

\section{Descriptive Differences Teachers}

The differences between the T-In and the T-Out in percent and rate are described below. The key words and definitions for the teacher's elements can be found in Appendix A. The descriptions of differences in the data pertain to Table 3 and Table 4.

Table 3-Element Data Summary T-In

\begin{tabular}{|c|c|c|c|c|c|c|c|c|}
\hline Key & Category & Count & Mean Time & Std.Dev. & Percentage & Rate & Duration & True \% \\
\hline (1)-1 & General Observation & 42 & 2.383 & 1.855 & 1.293 & 1.650 & 100.254 & $6.56 \%$ \\
\hline$(2)-2$ & Positive Feedback: Specific & 53 & 0.820 & 0.625 & 0.565 & 2.082 & 43.828 & $2.87 \%$ \\
\hline (3)-3 & Positive Feedback: General & 14 & 1.719 & 1.348 & 0.311 & 0.550 & 24.082 & $1.58 \%$ \\
\hline (4)-4 & Corrective Feedback: Skill & 5 & 1.016 & 0.566 & 0.065 & 0.196 & 5.059 & $0.33 \%$ \\
\hline (6)-6 & Management: General & 30 & 6.953 & 14.160 & 2.688 & 1.178 & 208.398 & $13.64 \%$ \\
\hline (7)-7 & Management: Direction & 98 & 2.637 & 2.617 & 3.328 & 3.849 & 258.027 & $16.89 \%$ \\
\hline
\end{tabular}




\begin{tabular}{|c|c|c|c|c|c|c|c|c|}
\hline$(8)-8$ & Verbal Instruction: Skill Explanation & 53 & 3.379 & 3.418 & 2.304 & 2.082 & 178.594 & $11.69 \%$ \\
\hline$(9)-9$ & Verbal Instruction: Prompt & 125 & 0.938 & 1.484 & 1.498 & 4.910 & 116.152 & $7.60 \%$ \\
\hline$(10)-0$ & Verbal Instruction: Relating Statement & 12 & 4.512 & 4.102 & 0.697 & 0.471 & 54.043 & $3.54 \%$ \\
\hline (11)-A & Context: Review & 2 & 102.148 & 101.367 & 2.635 & 0.079 & 204.277 & $13.37 \%$ \\
\hline (14)-D & Context: Music Off & 8 & 147.188 & 131.250 & 15.187 & 0.314 & 1177.480 & $77.06 \%$ \\
\hline (15)-E & Physical Contact: Positive & 3 & 0.840 & 1.406 & 0.032 & 0.118 & 2.520 & $0.16 \%$ \\
\hline$(16)-\mathrm{F}$ & Context: Teacher Position Central & 27 & 44.863 & 48.301 & 15.621 & 1.061 & 1211.113 & $79.26 \%$ \\
\hline (17)-G & Context: Teacher Position Periphery & 25 & 14.922 & 23.320 & 4.812 & 0.982 & 373.105 & $24.42 \%$ \\
\hline (18)-H & Context: Transition & 21 & 2.598 & 2.813 & 0.702 & 0.825 & 54.395 & $3.56 \%$ \\
\hline (19)-I & Teacher Questioning: Subject Matter & 31 & 2.188 & 1.367 & 0.875 & 1.218 & 67.852 & $4.44 \%$ \\
\hline (20)-J & Context: Indecision & 4 & 2.871 & 1.309 & 0.148 & 0.157 & 11.465 & $0.75 \%$ \\
\hline$(21)-K$ & Context: Pupil Configuration Warm Up & 3 & 72.461 & 23.750 & 2.804 & 0.118 & 217.363 & $14.23 \%$ \\
\hline$(22)-\mathrm{L}$ & Context: Pupil Configuration Center & 5 & 102.832 & 116.055 & 6.631 & 0.196 & 514.121 & $33.65 \%$ \\
\hline$(25)-\mathrm{O}$ & Context: Lesson Preview & 1 & 498.496 & 1.504 & 6.430 & 0.039 & 498.496 & $32.62 \%$ \\
\hline (26)-P & Context: Instruction & 1 & 849.336 & 0.176 & 10.955 & 0.039 & 849.336 & $55.58 \%$ \\
\hline (27)-Q & Verbal Instruction: Strategies/Rules & 35 & 2.754 & 1.621 & 1.239 & 1.375 & 96.055 & $6.29 \%$ \\
\hline$(28)-\mathrm{R}$ & Off Task & 1 & 48.730 & 0.176 & 0.629 & 0.039 & 48.73 & $3.19 \%$ \\
\hline (29)-S & Context: Music On & 8 & 46.934 & 14.023 & 4.843 & 0.314 & 375.449 & $24.57 \%$ \\
\hline$(30)-\mathrm{T}$ & Specific Observation & 71 & 0.938 & 0.977 & 0.861 & 2.789 & 66.758 & $4.37 \%$ \\
\hline$(31)-U$ & Teacher Answers & 13 & 4.531 & 3.613 & 0.761 & 0.511 & 59.023 & $3.86 \%$ \\
\hline (33)-W & Modeling & 35 & 3.926 & 4.785 & 1.770 & 1.375 & 137.207 & $8.98 \%$ \\
\hline$(35)-Y$ & Confusing Statement & 4 & 1.738 & 0.840 & 0.090 & 0.157 & 6.973 & $0.46 \%$ \\
\hline$(36)-Z$ & Context: Pupil Configuration Drillwork & 2 & 396.445 & 445.859 & 10.227 & 0.079 & 792.91 & $51.89 \%$ \\
\hline
\end{tabular}

Note. Total Elements=732, Total Time $=25.46$ minutes. Elemental Velocity $=(\mathrm{TE} / \mathrm{TT})=(732 / 25.46 \mathrm{mins})=$.28.75 elements per. $\mathrm{min}$.

Table 4-Element Data Summary T-Out

\begin{tabular}{|l|l|l|l|l|l|l|l|l|}
\hline Key & Category & Count & Mean Time & Std.Dev. & Percentage & Rate & Duration & True \% \\
\hline$(1)-1$ & General Observation & 64 & 2.773 & 1.836 & 2.195 & 2.425 & 177.871 & $10.66 \%$ \\
\hline$(2)-2$ & Positive Feedback: Specific & 62 & 0.508 & 0.371 & 0.393 & 2.349 & 31.875 & $1.91 \%$ \\
\hline$(3)-3$ & Positive Feedback: General & 5 & 0.801 & 0.625 & 0.050 & 0.189 & 4.023 & $0.24 \%$ \\
\hline$(4)-4$ & Corrective Feedback: Skill & 7 & 1.719 & 2.227 & 0.148 & 0.265 & 11.992 & $0.72 \%$ \\
\hline$(5)-5$ & Corrective Feedback: Negative Behavior & 12 & 3.145 & 2.715 & 0.467 & 0.455 & 37.823 & $2.27 \%$ \\
\hline$(6)-6$ & Management General & 35 & 2.773 & 2.246 & 1.200 & 1.326 & 97.285 & $5.83 \%$ \\
\hline
\end{tabular}




\begin{tabular}{|c|c|c|c|c|c|c|c|c|}
\hline (7)-7 & Management Direction & 185 & 1.875 & 1.758 & 4.263 & 7.009 & 345.488 & $20.71 \%$ \\
\hline (8)-8 & Verbal Instruction: Skill Explanation & 39 & 3.691 & 2.656 & 1.774 & 1.478 & 143.770 & $8.62 \%$ \\
\hline (9)-9 & Verbal Instruction: Prompt & 114 & 1.211 & 1.133 & 1.692 & 4.319 & 137.148 & $8.22 \%$ \\
\hline$(10)-0$ & Verbal Instruction: Relating Statement & 20 & 2.109 & 1.855 & 0.522 & 0.758 & 42.305 & $2.54 \%$ \\
\hline (11)-A & Context: Review & 1 & 84.121 & 0.195 & 1.038 & 0.038 & 84.121 & $5.04 \%$ \\
\hline (14)-D & Context: Music Off & 6 & 95.078 & 132.402 & 7.039 & 0.227 & 570.527 & $34.20 \%$ \\
\hline (15)-E & Physical Contact: Positive & 2 & 1.875 & 2.305 & 0.047 & 0.076 & 3.770 & $0.23 \%$ \\
\hline$(16)-\mathrm{F}$ & Context: Teacher Position Central & 59 & 21.289 & 19.824 & 15.494 & 2.235 & 1255.781 & $75.29 \%$ \\
\hline (17)-G & Context: Teacher Position Periphery & 60 & 6.836 & 7.441 & 5.054 & 2.273 & 409.59 & $24.56 \%$ \\
\hline (18)-H & Context: Transition & 35 & 1.367 & 1.191 & 0.594 & 1.326 & 48.105 & $2.88 \%$ \\
\hline (19)-I & Teacher Questioning: Subject Matter & 26 & 1.680 & 1.836 & 0.538 & 0.985 & 43.633 & $2.62 \%$ \\
\hline (20)-J & Context: Indecision & 10 & 3.379 & 2.168 & 0.418 & 0.379 & 33.848 & $2.03 \%$ \\
\hline$(21)-K$ & Context: Pupil Configuration Warm Up & 1 & 79.688 & 0.215 & 0.983 & 0.038 & 79.688 & $4.78 \%$ \\
\hline (22)-L & Context: Pupil Configuration Center & 2 & 178.340 & 135.078 & 4.401 & 0.076 & 356.680 & $21.38 \%$ \\
\hline$(25)-\mathrm{O}$ & Context: Lesson Preview & 1 & 354.355 & 1.621 & 4.372 & 0.038 & 354.355 & $21.24 \%$ \\
\hline (26)-P & Context: Instruction & 1 & 1228.125 & 0.176 & 15.153 & 0.038 & 1228.125 & $73.63 \%$ \\
\hline (27)-Q & Verbal Instruction: Strategies/Rules & 17 & 2.480 & 2.285 & 0.520 & 0.644 & 42.109 & $2.52 \%$ \\
\hline (28)-R & Off Task & 1 & 0.820 & 0.215 & 0.010 & 0.038 & 0.820 & $0.05 \%$ \\
\hline (29)-S & Context: Music On & 5 & 219.199 & 173.965 & 13.523 & 0.189 & 1096.016 & $65.71 \%$ \\
\hline$(30)-\mathrm{T}$ & Specific Observation & 75 & 0.273 & 0.313 & 0.258 & 2.841 & 20.918 & $1.25 \%$ \\
\hline (31)-U & Teacher Answers & 5 & 3.066 & 2.266 & 0.189 & 0.189 & 15.293 & $0.92 \%$ \\
\hline (33)-W & Modeling & 33 & 3.906 & 4.336 & 1.593 & 1.250 & 129.121 & $7.74 \%$ \\
\hline$(35)-\mathrm{Y}$ & Confusing Statement & 28 & 2.676 & 2.598 & 0.925 & 1.061 & 74.961 & $4.49 \%$ \\
\hline$(36)-Z$ & Context: Pupil Configuration Drillwork & 1 & 1227.656 & 0.234 & 15.147 & 0.038 & 1227.656 & $73.60 \%$ \\
\hline
\end{tabular}

Note. Total Elements $=912$, Total Time $=27.8$ minutes. Elemental Velocity $=(\mathrm{TE} / \mathrm{TT})=(912 / 27.8 \mathrm{mins})=$.32.80 elements per. min.

The percentage of class time devoted to the General Observation (1) element for the T-

Out was $10.66 \%$, while the percentage class time devoted to this element for the T-In was $6.56 \%$.

The rate or occurrence per minute by the T-Out was 2.425 , while the rate for the T-In was 1.650 .

The percentage of class time devoted to the General Observation (1) element was approximately

4\% higher in the T-Out and the rate was approximately double. However, in contrast, regarding

the element of Specific Observation (T), the T-In exhibited $4.37 \%$ of class time regarding this 
element, while the T-Out exhibited only $1.25 \%$ of class time. Consequently, when examining these two categories, the T-Out spent more time in the General Observation (1) element than the T-In and the T-In spent more time in the Specific Observation (T) element.

When we examined the T-In and the T-Out regarding the Corrective Feedback: Negative Behavior (5) element there was notable differences. The T-In had zero percent class time devoted to this element when compared with the T-Out at $2.27 \%$. Consequently, the T-In did not take up any class time for this element.

Regarding the Management: Direction (7) element, the T-In and the T-Out detailed differences with the percent and rate measurements. The T-In exhibited $16.89 \%$ class time regarding this element, while the T-Out exhibited $20.71 \%$, or almost $4 \%$ more class time spent in the Management: Direction (7) element.

The Verbal Instruction: Skill Explanation (8) element differed for the T-In and the T-Out. The percentage of class time devoted to this element for the T-In was $11.69 \%$, while the percentage for the T-Out was $8.62 \%$. This constituted a difference of approximately $3 \%$ regarding this element. The rate was also higher for the T-In at 2.082 versus 1.478 for the T-Out.

Regarding the Verbal Instruction: Strategies and Rules (Q) element, the percentage and rate for the two teachers, based on the class time devoted to this element, was different. The T-In exhibited $6.29 \%$ of this element, while the T-Out exhibited $2.52 \%$. The T-In exhibited double the percentage of time regarding this element as the T-Out. The rate was much higher for the T-In as well at two times that of the T-Out at 1.375 versus .644 .

Differences were exhibited in the contextual categories of preview, instruction and review for the T-In and the T-Out. In the Context: Lesson Preview (O) element the T-In exhibited 32.62\%, while the T-Out exhibited 21.24\%. In the Context: Instruction (P) element, the 
T-In exhibited 55.58\%, while the T-Out exhibited $73.63 \%$. The percentage of class time devoted to the Context: Review (A) element for the T-In was $13.37 \%$, while for the T-Out it was 5.04\%. Consequently, the T-In exhibited approximately $10 \%$ more class time in the Context: Lesson Preview (O) element and 8\% more class time in the Context: Lesson Review (A) element, while the T-Out exhibited 18\% more class time spent in the Context: Instruction (P) element.

Regarding the Teacher Questioning: Subject Matter (I) element, the T-In exhibited $4.44 \%$, for this element, while the T-Out exhibited $2.62 \%$. The rate or occurrence per minute was also higher for the T-In at 1.218 versus .985 for the T-Out. The T-In exhibited approximately double the class time in this element when compared with the time spent in this element for the T-Out.

The percentage of class time for the T-In versus the T-Out regarding the Context: Pupil Configuration Warm Up (K) element exhibited differences. The T-In exhibited $14.23 \%$ class time in this element, while the T-Out exhibited $4.78 \%$. Consequently, the T-In spent approximately $9 \%$ more class time in this element. Regarding another context element, the Context: Pupil Configuration Center (F) element, the T-In again exhibited a higher percentage of class time than the T-Out. The T-In exhibited $33.65 \%$ of class time in this element, while the TOut exhibited $21.38 \%$. The T-In exhibited approximately $12 \%$ more class time spent in this element than the T-Out.

The percentage of class time in the Off Task $(\mathrm{R})$ element was substantially different for each teacher. The T-In exhibited $3.19 \%$ of class time in this element, while the T-Out exhibited only $0.05 \%$ in this element. The difference in this element reflected the amount of time the T-In spent answering the phone call received from the office. 
Regarding the context elements of Context: Music On (S) and Context: Music Off (D), there was noticeable differences in class time for each element. Regarding the Context: Music On (S) element, the T-In exhibited $24.57 \%$ of class time in this element, while the T-Out exhibited 65.71\%. Consequently, regarding the Context: Music Off (D) element, the T-In exhibited $77.06 \%$ of class time in this element, while the T-Out exhibited $34.20 \%$. Regarding the context music elements, the T-Out exhibited approximately $41 \%$ more class time with the music on, while the T-In exhibited approximately $42 \%$ more class time with the music off.

In relation to the Teacher Answers (U) element there were differences regarding the T-In and the T-Out. Regarding this element, the T-In exhibited $3.86 \%$ of class time, while the T-Out exhibited $.92 \%$. There was almost four times less class time devoted to this element for the TOut.

When examining the Confusing Statement (Y) element, the T-Out exhibited a higher percentage of class time than the T-In regarding this element. The T-Out exhibited $4.49 \%$ class time involved in this element, while the T-In exhibited .46\%. This means that the T-Out spent approximately nine times more class time involved in this element than the T-In.

Regarding the amount of time spent in drillwork, T-In and T-Out differed substantially. The T-Out exhibited $73.60 \%$ class time in this element, while the T-In exhibited $51.89 \%$. The TOut exhibited approximately $21 \%$ more class time in the Context: Pupil Configuration Drillwork (Z) element than the T-In.

Summary of teacher behavior. The substantial differences in data between each teacher are summarized as follows. The T-Out spent more time in the General Observation (1) element, while the T-In spent more time in the Specific Observation (T) element. Regarding the Corrective Feedback: Negative Behavior (5) element, the T-Out exhibited this element, while the 
T-In did not. When examining the Management: Direction (7) element, more time was spent in this element by the T-Out than the T-In. The T-In exhibited more time in the Verbal Instruction: Skill Explanation (8) and the Verbal Instruction: Strategies and Rules (Q) elements than the TOut. The T-In spent more time in the Context: Lesson Preview (O) and Context: Lesson Review (A) elements and the T-Out spent more time in the Context: Instruction (P) element. The T-In spent more time than the T-Out in the Teacher Questioning: Subject Matter (I) element, and the Teacher Answers (U) element. The T-In spent more time in the Context: Pupil Configuration Warm Up (K) element as well as the Context: Pupil Configuration Central (F) element than the T-Out. The T-In exhibited more time in the Off Task (R) element than T-Out, but as a caveat to this was the office phone call received. The T-In spent more class time with the Context: Music Off (D) element and the T-Out spent more class time with the Context: Music On (S) element. The T-Out had more time spent in the Confusing Statement (Y) element than the T-In and spent more time in the Context: Pupil Configuration Drillwork $(Z)$ element than the T-In.

\section{Descriptive Differences Students}

The differences between the student's within-field (S-In) and the student's out-of-field (S-Out) in percent and rate are described below. The key words and definitions for the student's elements can be found in Appendix B. These descriptions of differences in the data pertain to Table 5 and Table 6.

Table 5-Element Data Summary S-In

\begin{tabular}{|l|l|l|l|l|l|l|l|l|}
\hline Key & Category & Count & Mean Time & Std.Dev. & Percentage & Rate & Duration & True \% \\
\hline$(1)-1$ & Motor Appropriate & 55 & 4.316 & 9.023 & 3.075 & 2.160 & 237.207 & $15.52 \%$ \\
\hline$(2)-2$ & Motor Inappropriate & 11 & 0.527 & 0.664 & 0.075 & 0.432 & 5.762 & $0.38 \%$ \\
\hline$(4)-4$ & Cognitive: Passive & 38 & 15.000 & 12.129 & 7.386 & 1.493 & 569.668 & $37.28 \%$ \\
\hline$(5)-5$ & Cognitive: Active & 17 & 1.563 & 1.289 & 0.343 & 0.668 & 26.484 & $1.73 \%$ \\
\hline$(6)-6$ & On Task Management: Verbal & 3 & 1.719 & 0.273 & 0.067 & 0.118 & 5.176 & $0.34 \%$ \\
\hline
\end{tabular}




\begin{tabular}{|c|c|c|c|c|c|c|c|c|}
\hline$(7)-7$ & On Task Management: Non-Verbal & 38 & 5.703 & 9.512 & 2.812 & 1.493 & 216.895 & $14.19 \%$ \\
\hline$(8)-8$ & Off Task & 9 & 4.297 & 3.086 & 0.500 & 0.354 & 38.594 & $2.53 \%$ \\
\hline $\begin{array}{l}(9)-9 \\
\end{array}$ & Interim & 5 & 4.199 & 2.773 & 0.272 & 0.196 & 20.977 & $1.37 \%$ \\
\hline$(10)-0$ & Waiting & 47 & 6.563 & 9.258 & 3.999 & 1.846 & 308.418 & $20.18 \%$ \\
\hline (11)-A & Context: Review & 2 & 102.148 & 101.367 & 2.649 & 0.079 & 204.277 & $13.37 \%$ \\
\hline (14)-D & Context: Music Off & 8 & 147.188 & 131.250 & 15.266 & 0.314 & 1177.480 & $77.06 \%$ \\
\hline (16)-F & Context: Teacher Position Central & 27 & 44.863 & 48.301 & 15.702 & 1.061 & 1211.113 & $79.26 \%$ \\
\hline$(17)-\mathrm{G}$ & Context: Teacher Position Periphery & 25 & 14.922 & 23.320 & 4.837 & 0.982 & 373.105 & $24.42 \%$ \\
\hline$(18)-\mathrm{H}$ & Context: Transition & 21 & 2.598 & 2.813 & 0.705 & 0.825 & 54.395 & $3.56 \%$ \\
\hline (20)-J & Context: Indecision & 4 & 2.871 & 1.309 & 0.149 & 0.157 & 11.465 & $0.75 \%$ \\
\hline$(21)-K$ & Context: Pupil Configuration Warm Up & 3 & 72.461 & 23.750 & 2.818 & 0.118 & 217.363 & $14.23 \%$ \\
\hline (22)-L & Context: Pupil Configuration Center & 5 & 102.832 & 116.055 & 6.666 & 0.196 & 514.121 & $33.65 \%$ \\
\hline$(25)-\mathrm{O}$ & Context: Lesson Preview & 1 & 498.496 & 1.504 & 6.463 & 0.039 & 498.496 & $32.62 \%$ \\
\hline (26)-P & Context: Instruction & 1 & 849.336 & 0.176 & 11.012 & 0.039 & 849.336 & $55.58 \%$ \\
\hline (29)-S & Context: Music On & 8 & 46.934 & 14.023 & 4.868 & 0.314 & 375.449 & $24.57 \%$ \\
\hline$(33)-W$ & Confusion & 2 & 2.090 & 0.605 & 0.054 & 0.079 & 4.180 & $0.27 \%$ \\
\hline$(36)-Z$ & Context: Pupil Configuration Drillwork & 2 & 396.445 & 445.859 & 10.280 & 0.079 & 792.910 & $51.89 \%$ \\
\hline
\end{tabular}

Note. Total Elements=332, Total Time $=25.46$ minutes. Elemental Velocity $=(\mathrm{TE} / \mathrm{TT})=(332 / 25.46 \mathrm{mins})=$.13.04 elements per. $\mathrm{min}$.

\section{Table 6-Element Data Summary S-Out}

\begin{tabular}{|l|l|l|l|l|l|l|l|l|}
\hline Key & Category & Count & Mean Time & StdDev & Percentage & Rate & Duration & True \% \\
\hline$(1)-1$ & Motor Appropriate & 18 & 6.758 & 5.059 & 1.472 & 0.682 & 121.719 & $7.30 \%$ \\
\hline$(2)-2$ & Motor Inappropriate & 17 & 2.305 & 4.531 & 0.473 & 0.644 & 39.102 & $2.34 \%$ \\
\hline$(3)-3$ & Motor Supporting & 8 & 5.488 & 7.500 & 0.531 & 0.303 & 43.926 & $2.63 \%$ \\
\hline$(4)-4$ & Cognitive: Passive & 26 & 16.699 & 23.535 & 5.250 & 0.985 & 434.141 & $26.03 \%$ \\
\hline$(5)-5$ & Cognitive: Active & 9 & 2.656 & 2.227 & 0.290 & 0.341 & 23.945 & $1.44 \%$ \\
\hline$(6)-6$ & On Task Management: Verbal & 2 & 2.461 & 2.051 & 0.060 & 0.076 & 4.922 & $0.30 \%$ \\
\hline$(7)-7$ & On Task Management: Non-Verbal & 18 & 5.547 & 4.355 & 1.209 & 0.682 & 100.000 & $6.00 \%$ \\
\hline$(8)-8$ & Off Task & 31 & 4.805 & 4.238 & 1.798 & 1.174 & 148.711 & $8.92 \%$ \\
\hline$(9)-9$ & Interim & 26 & 4.355 & 2.344 & 1.367 & 0.985 & 113.066 & $6.78 \%$ \\
\hline$(10)-0$ & Waiting & 37 & 13.164 & 11.758 & 5.894 & 1.402 & 487.402 & $29.22 \%$ \\
\hline$(11)-A$ & Context: Review & 1 & 84.121 & 0.488 & 1.017 & 0.038 & 84.121 & $5.04 \%$ \\
\hline$(14)-D$ & Context: Music Off & 6 & 95.078 & 132.402 & 6.899 & 0.227 & 570.527 & $34.20 \%$ \\
\hline$(15)-E$ & Negative Self-worth Comment & 1 & 5.547 & 1.602 & 0.067 & 0.038 & 5.547 & $0.33 \%$ \\
\hline
\end{tabular}




\begin{tabular}{|l|l|l|l|l|l|l|l|l|}
\hline$(16)-\mathrm{F}$ & Context: Teacher Position Central & 59 & 21.289 & 19.824 & 15.186 & 2.235 & 1255.781 & $75.29 \%$ \\
\hline$(17)-\mathrm{G}$ & Context: Teacher Position Periphery & 60 & 6.836 & 7.441 & 4.953 & 2.273 & 409.590 & $24.56 \%$ \\
\hline$(18)-\mathrm{H}$ & Context: Transition & 35 & 1.367 & 1.191 & 0.582 & 1.326 & 48.105 & $2.88 \%$ \\
& & & & & & & \\
\hline$(20)-\mathrm{J}$ & Context: Indecision & 10 & 3.379 & 2.168 & 0.409 & 0.379 & 33.848 & $2.03 \%$ \\
\hline$(21)-\mathrm{K}$ & Context: Pupil Configuration Warm Up & 1 & 79.688 & 0.215 & 0.964 & 0.038 & 79.688 & $4.78 \%$ \\
\hline$(22)-\mathrm{L}$ & Context: Pupil Configuration Center & 2 & 178.340 & 135.078 & 4.313 & 0.076 & 356.680 & $21.38 \%$ \\
\hline$(25)-\mathrm{O}$ & Context: Lesson Preview & 1 & 354.355 & 1.621 & 4.285 & 0.038 & 354.355 & $21.24 \%$ \\
\hline$(26)-\mathrm{P}$ & Context: Instruction & 1 & 1228.125 & 0.176 & 14.851 & 0.038 & 1228.125 & $73.63 \%$ \\
\hline$(29)-\mathrm{S}$ & Context: Music On & 5 & 219.199 & 173.965 & 13.254 & 0.189 & 1096.016 & $65.71 \%$ \\
\hline (33)-W & Confusion & 2 & 1.230 & 1.855 & 0.030 & 0.076 & 2.461 & $0.15 \%$ \\
\hline (36)-Z & Context: Pupil Configuration Drillwork & 1 & 1227.656 & 0.195 & 14.846 & 0.038 & 1227.656 & $73.60 \%$ \\
\hline
\end{tabular}

The percentage of class time devoted to the Motor Appropriate (1) element by the S-In was $15.52 \%$, while the percentage of class time devoted to this element was $7.30 \%$ for the S-Out. The rates for these students differed as well in that the S-In exhibited this element at a rate of 2.160 occurrences per minute, while the rate exhibited by the S-Out was .682 occurrences per minute. Consequently the S-In exhibited approximately $8 \%$ more class time devoted to the Motor Appropriate (1) element.

In regards to the Cognitive: Passive (4) element, substantial differences were exhibited between the S-In and the S-Out. The percentage of the Cognitive: Passive (4) element was $37.28 \%$ for the S-In, while the percentage was $26.03 \%$ for the S-Out. The difference in percentage class time regarding this element was approximately $11 \%$ higher for the S-In. The rate also differed slightly as well with the S-In exhibiting this element at 1.4 occurrences per minute, while the S-Out exhibited this element at .985 occurrences per minute.

The percentage of the S-In class time devoted to the On Task Management: Non-Verbal (7) element was $14.19 \%$, while the percentage for this element was $6.00 \%$ for the S-Out. The rate for the S-In and the S-Out differed as well with the S-In exhibiting 1.493 occurrences per minute, 
while the S-Out exhibited this element at .682 occurrences per minute. The S-In exhibited this element by approximately $8 \%$ more class time when compared to the S-Out and had more occurrences per minute.

Regarding the Off Task (8) element, the S-Out exhibited a larger percentage of time in this element than the S-In. The S-Out exhibited $8.92 \%$ class time devoted to this element, while the S-In exhibited $2.53 \%$. This is a difference of approximately $6 \%$ more class time exhibited by the S-Out than the S-In regarding this element. The rate differed as well with the S-Out exhibiting 1.174 occurrences per minute, while the S-In exhibited .354 occurrences per minute. The S-Out therefore exhibited this element in more times per minute and more total time.

Regarding the element of Interim (9), the S-Out exhibited more of this element than the S-In. The S-Out exhibited this element with $6.78 \%$ class time devoted to this element, while the S-In exhibited $1.37 \%$. This is approximately a $5 \%$ difference regarding class time devoted to this element. The rate differed as well with the S-Out exhibiting .985 occurrences per minute, while the S-In exhibited .196 occurrences per minute.

When the Waiting (0) element was examined for the S-Out and the S-In, substantial differences were exhibited. The S-Out exhibited $29.22 \%$ of class time devoted to this element, while the S-In exhibited this element in $20.18 \%$ of their class time. Basically, the S-Out waited for $9 \%$ more class time than the S-In. The rate of occurrence however was higher for the S-In versus the S-Out at 1.846 occurrences per minute versus the 1.402 for the S-Out. Consequently the S-In had more occurrences of waiting per minute, but the total class time or duration of this element was 9\% less for the S-In. This equates to more occurrences per minute for the S-In, but less class time spent in this element regarding each occurrence. 
The amount of time spent in the Context: Pupil Configuration Drillwork (Z) element by the S-In was far less than the S-Out. The S-Out spent $73.60 \%$ in this element, while the S-In spent $51.89 \%$ of class time in this element. This element was exhibited by approximately $22 \%$ less class time for the S-In.

Summary of student behavior. The substantial differences in data between the S-In and the S-Out are summarized below. The S-In spent more time in the Motor Appropriate (1) element than the S-Out. The S-In spent more time in the Cognitive: Passive (4) element than the S-Out. The S-In exhibited more of the On Task Management: Non-Verbal (7) element than the S-Out. The S-Out exhibited more of the Off Task (8) element than the S-In. The S-Out exhibited more of the Interim (9) element than the S-In. The S-Out exhibited more of the Waiting (0) element than the S-In. Regarding all of these elements, for time spent involved in drillwork, the S-Out spent more time in this category than the S-In.

Even though the students had to wait longer during the time the teacher was involved in the Off Task element as he answered the phone, the S-In still exhibited less class time in the Waiting element. The S-In also exhibited more class time in the Motor Appropriate (1) element, while spending substantially less time involved in the Context: Pupil Configuration Drillwork (Z) element than the S-Out.

\section{Sequence Differences - Teachers}

Two event sequence. The differences between the T-In and the T-Out in the two event sequence chains are described below. Abbreviations of key words for the teacher's categories are in Table 7. 


\section{Table 7-Teacher Elements Alpha-numeric Key}

\begin{tabular}{|c|c|}
\hline Element & Abbreviation (Chains) \\
\hline 1. General Observation (behavioral element) & Gen. Ob.(1) \\
\hline 2. Positive Feedback: Specific (behavioral element) & Pos. Fed: Spec.(2) \\
\hline 3. $\quad$ Positive Feedback: General (behavioral element) & Pos. Fed: Gen.(3) \\
\hline 4. $\quad$ Corrective Feedback: Skill (behavioral element) & Cor. Fed: Skil.(4) \\
\hline 5. Corrective Feedback: Negative Behavior (behavioral element) & Cor. Fed: Neg. B.(5) \\
\hline 6. Management: General (behavioral element) & Man: Gen.(6) \\
\hline 7. Management: Direction (behavioral element) & Man: Dir.(7) \\
\hline 8. Verbal Instruction: Skill Explanation (behavioral element) & VI: Ski. Exp.(8) \\
\hline 9. Verbal Instruction: Prompt (behavioral element) & VI: Pmpt.(9) \\
\hline 10. (0) Verbal Instruction: Relating Statement (behavioral element) & VI: Rel. Stat.(0) \\
\hline 11. (A) Context: Review (contextual element) & Cxt: Rev.(A) \\
\hline $12 . \quad-$ & - \\
\hline 13. - & - \\
\hline 14. (D) Context: Music Off (contextual element) & Cxt: Mus. Of.(D) \\
\hline 15. (E) Physical Contact: Positive (behavioral element) & Phys. Cont: Pos.(E) \\
\hline 16. (F) Context: Teacher Position Central (contextual element) & Cxt: Tchr. Cen.(F) \\
\hline 17. (G) Context: Teacher Position Periphery (contextual element) & Cxt: Tchr. Per.(G) \\
\hline 18. (H) Context: Transition (contextual element) & Cxt: Trans.(H) \\
\hline 19. (I) Teacher Questioning: Subject Matter (behavioral element) & TQ: Sub. Mat.(I) \\
\hline 20. (J) Context: Indecision (contextual element) & Cxt: Indec.(J) \\
\hline 21. (K) Context: Pupil Configuration Warm Up (contextual element) & Cxt: Pup. Conf. Wrm. Up.(K) \\
\hline 22. (L) Context: Pupil Configuration Center (contextual element) & Cxt: Pup. Conf. Cen.(L) \\
\hline $23 . \quad-$ & - \\
\hline $24 . \quad-$ & - \\
\hline 25. (O) Context: Lesson Preview (contextual element) & Cxt: Les. Prev.(O) \\
\hline 26. (P) Context: Instruction (contextual element) & Cxt: Instr.(P) \\
\hline 27. (Q) Verbal Instruction: Strategies/Rules (behavioral element) & VI: Str./Rul.(Q) \\
\hline 28. (R) Off Task (behavioral element) & Of. Tsk.(R) \\
\hline 29. (S) Context: Music On (contextual element) & Cxt: Mus. On(S) \\
\hline 30. (T) Specific Observation (behavioral element) & Spec. Ob.(T) \\
\hline 31. (U) Teacher Answers (behavioral element) & Tchr. Answr.(U) \\
\hline
\end{tabular}




\begin{tabular}{|c|l|}
\hline $32 . \quad-$ & - \\
\hline $33 . \quad$ (W) Modeling (behavioral element) & Model.(W) \\
\hline $34 . \quad-$ & - \\
\hline $35 . \quad(Y)$ Confusing Statement (behavioral element) & Confus. Stat.(Y) \\
\hline $36 . \quad($ Z) Context: Pupil Configuration Drillwork (contextual element) & Cxt: Pup. Conf. Dril.(Z) \\
\hline
\end{tabular}

Tables 8 and 9 delineate the chains for each teacher regarding the two event sequences. 
Table 8-Summary Two Event Sequence T-In

\begin{tabular}{|c|c|c|c|c|c|c|c|}
\hline Trigger & Successor & Freq./Prob. & Z-score & Trigger & Successor & Freq./Prob. & Z-score \\
\hline \multirow[t]{4}{*}{ Gen. Ob.(1) } & Spec. Ob.(T) & $9 / .0123$ & $2.555++$ & Cxt: Trans.(H) & Man: Dir.(7) & $7 / .0096$ & $2.672+++$ \\
\hline & Man: Gen.(6) & $8 / .0110$ & $4.872+++$ & & Cxt: Mus. On(S) & $4 / .0055$ & $7.896+++$ \\
\hline & Pos. Fed: Gen.(3) & $3 / .0041$ & $2.466++$ & & Cxt: Mus. Of.(D) & $3 / .0041$ & $5.801+++$ \\
\hline & & & & & Cxt: Tchr. Per.(G) & $3 / .0041$ & $2.734+++$ \\
\hline \multirow[t]{2}{*}{ Pos. Fed: Spec.(2) } & Spec. Ob.(T) & $12 / .0165$ & $3.168+++$ & & & & \\
\hline & & & & TQ: Sub. Mat.(I) & VI: Skil. Exp.(8) & $9 / .0123$ & $4.667+++$ \\
\hline \multirow[t]{2}{*}{ Cor. Fed: Skil. (4) } & VI: Str./Rul.(Q) & $3 / .0041$ & $5.773+++$ & & VI: Str./Rul.(Q) & $6 / .0082$ & $3.790+++$ \\
\hline & & & & & TQ: Sub. Mat.(I) & $4 / .0055$ & $2.387++$ \\
\hline \multirow[t]{4}{*}{ Man: Gen.(6) } & Gen. Ob.(1) & $8 / .0110$ & $4.914+++$ & & & & \\
\hline & Cxt: Trans.(H) & $8 / .0110$ & $7.789+++$ & Cxt: Indec.(J) & Confus. Stat.(Y) & $3 / .0041$ & $20.157+++$ \\
\hline & Cxt: Tchr. Per.(G) & $4 / .0055$ & $2.981+++$ & & & & \\
\hline & & & & VI: Str./Rul.(Q) & VI: Str./Rul.(Q) & $9 / .0123$ & $5.787+++$ \\
\hline \multirow[t]{4}{*}{ Man: Dir.(7) } & Man: Dir.(7) & $39 / .0535$ & $7.648+++$ & & TQ: Sub. Mat.(I) & $7 / .0096$ & $4.617+++$ \\
\hline & Man: Gen.(6) & $8 / .0110$ & $2.017++$ & & & & \\
\hline & Cxt: Indec.(J) & $3 / .0041$ & $3.367+++$ & Spec. Ob.(T) & Pos. Fed: Spec.(2) & $25 / .0343$ & $9.068+++$ \\
\hline & & & & & VI: Pmpt.(9) & $25 / .0343$ & $4.038+++$ \\
\hline \multirow[t]{4}{*}{ VI: Skil. Exp.(8) } & VI: Skil. Exp.(8) & $12 / .0165$ & $4.310+++$ & & & & \\
\hline & TQ: Sub. Mat.(I) & $11 / .0151$ & $5.954+++$ & Tchr. Answr. (U) & VI: Rel. Stat.(0) & $6 / .0082$ & $12.612+++$ \\
\hline & Model.(W) & $8 / .0110$ & $3.505+++$ & & & & \\
\hline & & & & Model.(W) & VI: Skil. Exp.(8) & $10 / .0137$ & $4.853+++$ \\
\hline \multirow[t]{2}{*}{ VI: Pmpt.(9) } & VI: Pmpt.(9) & $57 / .0782$ & $8.440+++$ & & Model.(W) & $5 / .0069$ & $2.625+++$ \\
\hline & Spec. Ob.(T) & $22 / .0302$ & $2.964+++$ & & & & \\
\hline
\end{tabular}




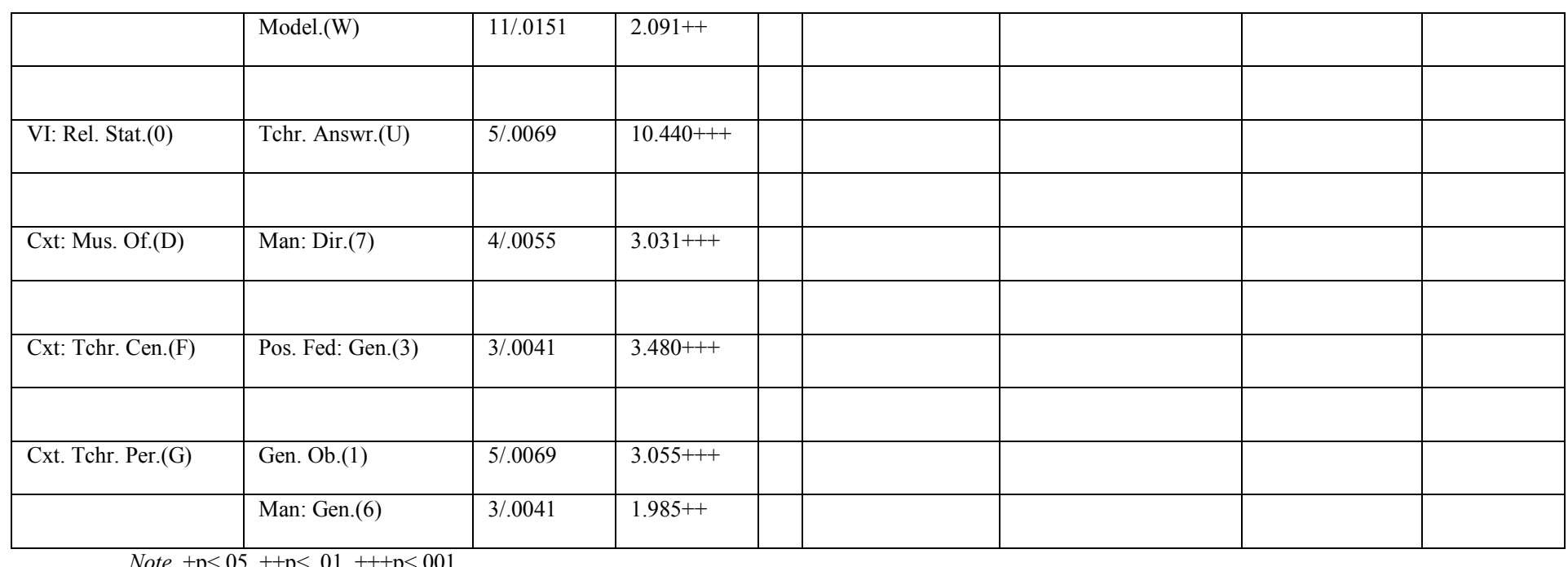

Note. $+\mathrm{p}<.05,++\mathrm{p}<. .01,+++\mathrm{p}<.001$

Rate $=(372 / 25.46$ minutes $)=14.61$ two event sequence chains per. min.

Coherence $=($ type $/$ freq. $/$ prob. $)=37 / 372 / .5104$

\section{Table 9-Summary Two Event Sequence T-Out}

\begin{tabular}{|c|c|c|c|c|c|c|c|}
\hline Trigger & Successor & Freq./Prob. & Z-score & Trigger & Successor & Freq./Prob. & Z-score \\
\hline \multirow[t]{3}{*}{ Gen. Ob.(1) } & Cxt: Tchr. Per.(G) & $19 / .0209$ & $7.455+++$ & Cxt. Tchr. Per.(G) & Gen. Ob.(1) & $14 / .0154$ & $4.938+++$ \\
\hline & Cxt: Tchr. Cen.(F) & $8 / .0088$ & $1.955+$ & & Spec. Ob.(T) & $9 / .0099$ & $1.904+$ \\
\hline & & & & & Man: Gen.(6) & $5 / .0055$ & $1.807+$ \\
\hline \multirow[t]{3}{*}{ Pos. Fed: Spec.(2) } & Pos. Fed: Spec.(2) & $10 / .0110$ & $2.911+++$ & & & & \\
\hline & VI: Str./Rul.(Q) & $4 / .0044$ & $2.666+++$ & Cxt: Trans.(H) & Man: Dir.(7) & $21 / .0231$ & $5.832+++$ \\
\hline & & & & & Cxt: Mus. Of.(D) & $3 / .0033$ & $5.784+++$ \\
\hline \multirow[t]{2}{*}{ Cor. Fed: Skil. (4) } & VI: Pmpt.(9) & $3 / .0033$ & $2.424+++$ & & & & \\
\hline & & & & TQ: Sub. Mat.(I) & VI: Skil. Exp.(8) & $3 / .0033$ & $1.826+$ \\
\hline Cor. Fed: Neg. B.(5) & Man: Dir.(7) & $5 / .0055$ & $1.837+$ & & TQ: Sub. Mat.(I) & $3 / .0033$ & $2.657+++$ \\
\hline
\end{tabular}




\begin{tabular}{|c|c|c|c|c|c|c|c|}
\hline & Gen. Ob.(1) & $3 / .0033$ & $2.434+$ & & VI: Str./Rul.(Q) & $3 / .0033$ & $3.642+++$ \\
\hline \multirow[t]{5}{*}{ Man: Gen.(6) } & Cxt: Tchr. Per.(G) & $5 / .0055$ & $1.906+$ & VI: Str./Rul.(Q) & Spec. Ob.(T) & $4 / .0044$ & $2.292++$ \\
\hline & Cxt: Trans.(H) & $5 / .0055$ & $3.293+++$ & & Pos. Fed: Spec.(2) & $3 / .0033$ & $1.773+$ \\
\hline & Gen. Ob.(1) & $5 / .0055$ & $1.750+$ & & & & \\
\hline & Man: Gen.(6) & $4 / .0044$ & $2.401++$ & Spec. Ob.(T) & Pos. Fed: Spec.(2) & $25 / .0275$ & $9.115+++$ \\
\hline & & & & & VI: Pmpt.(9) & $19 / .0209$ & $3.350+++$ \\
\hline \multirow[t]{5}{*}{ Man: Dir.(7) } & Cxt: Trans.(H) & $23 / .0253$ & $6.073+++$ & & Cxt: Tchr. Cen.(F) & $9 / .0099$ & $1.940+$ \\
\hline & Man: Gen.(6) & $15 / .0165$ & $3.014+++$ & & Cor. Fed: Skil.(4) & $3 / .0033$ & $3.202+++$ \\
\hline & Cor. Fed. Neg. B.(5) & $9 / .0099$ & $4.228+++$ & & & & \\
\hline & Cxt: Indec.(J) & $6 / .0066$ & $2.798+++$ & Model.(W) & VI: Skil. Exp.(8) & $9 / .0099$ & $6.520+++$ \\
\hline & & & & & VI: Rel. Stat.(0) & $5 / .0055$ & $5.076+++$ \\
\hline \multirow[t]{3}{*}{ VI: Skil. Exp.(8) } & Model.(W) & $11 / .0121$ & $8.211+++$ & & Confus. Stat.(Y) & $3 / .0033$ & $2.001++$ \\
\hline & VI: Skil. Exp.(8) & $7 / .0077$ & $4.213+++$ & & & & \\
\hline & & & & Confus. Stat.(Y) & TQ: Sub. Mat.(I) & $4 / .0044$ & $3.630+++$ \\
\hline \multirow[t]{3}{*}{ VI: Pmpt.(9) } & VI: Pmpt. (9) & $38 / .0418$ & $6.711+++$ & & & & \\
\hline & Model.(W) & $8 / .0088$ & $1.937+$ & & & & \\
\hline & VI: Str./Rul.(Q) & $5 / .0055$ & $1.986++$ & & & & \\
\hline VI: Rel. Stat.(0) & TQ: Sub. Mat.(I) & $3 / .0033$ & $3.260+++$ & & & & \\
\hline Cxt: Tchr. Cen.(F) & Man: Dir.(7) & $26 / .0286$ & $4.531++$ & & & & \\
\hline
\end{tabular}

Note. $+\mathrm{p}<.05,++\mathrm{p}<. .01,+++\mathrm{p}<.001$

$*$ Rate $=(367 / 27.8$ mins. $)=13.20$ two event sequence chains per. min.

$*$ Coherence $=($ type/freq. $/$ prob. $)=40 / 367 / .4037$ 
When the T-In data was examined, the most numerous chains, a cluster of four, came from the trigger of the Context: Transition $(\mathrm{H})$ element. The second two of the most numerous chains, a series of three clusters or chains coming after a trigger for the T-In dealt with the verbal instruction elements. These successors, coming after these verbal instruction triggers, were all conceptually related (see Appendix A for definitions). Each of these chains regarding this trigger therefore were instructionally relevant. For example, as a trigger, the Verbal Instruction: Skill Explanation (8) element had successors of which were each instructionally relevant. These were the Verbal Instruction: Skill Explanation (8) element, the Teacher Questioning: Subject Matter (I) element, and the Modeling (W) element. Therefore, the T-In exhibited one more conceptually related chain regarding an instructionally relevant element after the trigger of the Verbal Instruction: Skill Explanation (8) element than the T-Out.

Another interesting difference between the two teacher's lessons regarding the two event sequence chains was that after the General Observation (1) trigger, the element of Specific Observation (T) followed this trigger for the T-In. This was not the case for the T-Out.

When examining the Management: Direction (7) element, the T-In had one less type of chain that followed the trigger of this element than the T-Out. This chain dealt with the successor of the Corrective Feedback: Negative Behavior (5) element, which was not evident as a trigger or successor in any of the T-In chains. Consequently, the T-Out exhibited more chains with the trigger of the Management: Direction (7) element.

In examining the most common chains for the T-Out, there were chains that included clusters of four, such as the Context: Transition $(\mathrm{H})$ element acting as a trigger most numerously for the T-In. These clusters, or two event sequence chains with a trigger and four successors that 
followed, had the triggers of: the Management: General (6) element, the Management: Direction (7) element and the Specific Observation (T) element.

When the Modeling (W) element acted as trigger in the two event sequence chains, both teachers had similar successors as each had two instructionally relevant successors except that the T-Out exhibited a third chain in the cluster that the T-In did not. This third chain involved the Confusing Statement (Y) element. This detailed the existence of elements that followed the Modeling (W) element for the T-Out, which were instructionally and non-instructionally relevant.

When the element of Specific Observation (T) was examined as a trigger, each of the teacher's exhibited similar yet different data. The T-Out had four chains exhibited with three out of the four successors being instructionally relevant, and one having a contextual focus. One of the successors out of the three instructionally relevant ones was the Corrective Feedback: Skill (4) element for the T-Out. The T-In did not exhibit this Corrective Feedback Skill (4) element as a successor and did not exhibit any contextual elements in relation to the Specific Observation (T) element as a trigger. The T-In had two successors following the Specific Observation (1) element as a trigger and both were instructionally relevant.

Another notable difference between the two teacher's lessons regarding the two event sequence chains was that the T-In exhibited the Teacher Answers (U) element as a trigger and as a successor in the chains and the T-Out did not.

In summation. The number of two event sequence chains for each teacher with the most successors or clusters that followed one trigger was different for each teacher. The T-Out exhibited more complex chains or larger clusters, citing three different chains with a trigger that had four successors. However, two out of the three of these chains exhibited managerial triggers. 
The one cluster that had an instructionally relevant trigger, the Specific Observation (T) element, had a mixture of successors that were both instructionally and contextually focused. The T-Out exhibited the Corrective Feedback: Skill (4) element after this successor as one of the instructionally relevant elements, but this element was not exhibited by the T-In. The T-In exhibited this Specific Observation (T) element as a trigger, but with only instructionally relevant successors after this trigger in their data and no contextual elements. This evidence for greater predictability amongst the two event sequence chains was more common for the T-In.

When examining the T-In data, the T-In exhibited the same two event sequence chains with three successors for each trigger for the instructionally relevant elements as the T-Out. The difference between the teachers when examining these trigger and successor chains was that one out of the three chains for the T-Out had an element successor that was not instructionally relevant. This was the Confusing Statement (Y) element. Furthermore, when examining the data, the T-Out had chains with non-instructional element triggers including the Corrective Feedback: Negative Behavior (5) and the Confusing Statement (Y) elements. These triggers were not evident in the two event sequences for the T-In.

Regarding the five-second lag that was put into place to determine if time constraints made much of a difference in the teacher's two event sequence chains exhibited, there were not substantial differences within the two teacher's data.

Three event sequence. The differences between the T-In and the T-Out in the three event sequence chains have been described. Tables 10 and1 1 have delineated the chains for each teacher regarding the three event sequences. 
Table 10-Summary Three Event Sequence T-In

\begin{tabular}{|c|c|c|c|c|c|c|c|c|c|}
\hline Precursor & Fulcrum & Successor & Freq./Prob. & Z-score & Precursor & Fulcrum & Successor & Freq./Prob. & Z-score \\
\hline Man: Gen.(6) & Gen. Ob.(1) & Man: Gen.(6) & $3 / .0041$ & $4.750+++$ & VI: Pmpt.(9) & Spec.Ob.(T) & VI:Pmpt.(9) & $12 / .0165$ & $4.648+++$ \\
\hline \multirow[t]{2}{*}{ Pos. Fed: Spec.(2) } & Gen. Ob.(1) & Spec. Ob.(T) & $3 / .0041$ & $3.787+++$ & VI: Pmpt.(9) & Spec.Ob. (T) & Pos. Fed: Spec.(2) & $6 / .0082$ & $3.609+++$ \\
\hline & & & & & Gen. Ob.(1) & Spec. Ob.(T) & Pos. Fed: Spec.(2) & $5 / .0069$ & $5.574+++$ \\
\hline Spec. Ob.(T) & Pos. Fed: Spec.(2) & VI: Pmpt.(9) & $8 / .0110$ & $1.966++$ & Pos. Fed: Spec.(2) & Spec. Ob.(T) & VI: Pmpt.(9) & $5 / .0069$ & $2.250++$ \\
\hline Spec. Ob.(T) & Pos. Fed: Spec.(2) & Spec. Ob.(T) & $5 / .0069$ & $1.727+$ & Pos. Fed: Spec.(2) & Spec. Ob.(T) & Pos. Fed: Spec.(2) & $4 / .0055$ & $3.474+++$ \\
\hline \multirow[t]{2}{*}{ VI: Pmpt.(9) } & Pos. Fed: Spec. (2) & Spec. Ob.(T) & $3 / .0041$ & $2.384++$ & & & & & \\
\hline & & & & & VI: Pmpt.(9) & Model.(W) & VI: Pmpt.(9) & $5 / .0069$ & $2.487++$ \\
\hline Man: Dir.(7) & Man: Gen.(6) & Cxt: Trans.(H) & $3 / .0041$ & $5.850+++$ & Pos. Fed: Spec.(2) & Model.(W) & VI: Skil. Exp.(8) & $3 / .0041$ & $5.213+++$ \\
\hline Gen. Ob.(1) & Man: Gen.(6) & Gen. Ob.(1) & $3 / .0041$ & $3.849+++$ & VI: Skil. Exp.(8) & Model.(W) & Model.(W) & $3 / .0041$ & $4.322+++$ \\
\hline Man: Dir.(7) & Man: Dir.(7) & Man: Dir.(7) & $18 / .0247$ & $5.982+++$ & & & & & \\
\hline Man: Dir.(7) & Man: Dir.(7) & Man: Gen.(6) & $5 / .0069$ & $2.733+++$ & & & & & \\
\hline Cxt: Mus. Of.(D) & Man: Dir.(7) & Man: Dir.(7) & $3 / .0041$ & $3.606+++$ & & & & & \\
\hline VI: Skil. Exp.(8) & VI: Skil. Exp.(8) & VI: Skil. Exp.(8) & $6 / .0082$ & $5.696+++$ & & & & & \\
\hline TQ: Sub. Mat.(I) & VI: Skil. Exp.(8) & TQ: Sub. Mat.(I) & $4 / .0055$ & $5.971+++$ & & & & & \\
\hline Model.(W) & VI: Skil. Exp.(8) & Model.(W) & $3 / .0041$ & $3.724+++$ & & & & & \\
\hline VI: Pmpt.(9) & VI: Pmpt.(9) & VI: Pmpt.(9) & $30 / .0412$ & $7.099+++$ & & & & & \\
\hline Spec. Ob.(T) & VI: Pmpt.(9) & VI: Pmpt.(9) & $15 / .0206$ & $5.678+++$ & & & & & \\
\hline VI:Pmpt.(9) & VI: Pmpt.(9) & Spec. Ob.(T) & $11 / .0151$ & $2.429++$ & & & & & \\
\hline Pos. Fed: Spec.(2) & VI: Pmpt.(9) & VI: Pmpt.(9) & $6 / .0082$ & $3.287+++$ & & & & & \\
\hline
\end{tabular}




\begin{tabular}{|l|l|l|l|l|l|l|l|l|l|}
\hline Model.(W) & VI: Pmpt.(9) & Model.(W) & $4 / .0055$ & $5.558+++$ & & & & \\
\hline Model.(W) & VI: Pmpt.(9) & Spec. Ob.(T) & $3 / .0041$ & $2.384++$ & & & & \\
\hline & & & & & & & & & \\
\hline Man: Gen.(6) & Cxt: Trans.(H) & Cxt: Mus. On(S) & $3 / .0041$ & $9.876+++$ & & & & \\
\hline Man: Gen.(6) & Cxt: Trans.(H) & Cxt: Tchr. Per.(G) & $3 / .0041$ & $5.291+++$ & & & & \\
\hline & & & & & & & & \\
\hline VI: Str./Rul.(Q) & VI: Str./Rul.(Q) & TQ: Sub. Mat.(I) & $4 / .0055$ & $5.971+++$ & & & \\
\hline TQ: Sub. Mat.(I) & \multicolumn{2}{|l|}{ VI: Str./Rul.(Q) } & VI: Str./Rul.(Q) & $4 / .0055$ & $7.083+++$ & & & & \\
\hline
\end{tabular}

Note. $+\mathrm{p}<.05,++\mathrm{p}<. .01,+++\mathrm{p}<.001$ 
Table 11-Summary Three Event Sequence T-Out

\begin{tabular}{|c|c|c|c|c|c|c|c|c|c|}
\hline Precursor & Fulcrum & Successor & Freq./Prob. & Z-score & Precursor & Fulcrum & Successor & Freq./Prob. & Z-score \\
\hline Man: Dir.(7) & Gen. Ob.(1) & Cxt: Tchr. Per.(G) & $8 / .0088$ & $7.290+++$ & Man: Dir.(7) & Cxt: Trans.(H) & Man: Dir.(7) & $13 / .0143$ & $4.308+++$ \\
\hline \multirow[t]{2}{*}{ Cxt: Tchr. Per.(G) } & Gen. Ob.(1) & Cxt: Tchr. Cen.(F) & $4 / .0044$ & $3.397+++$ & Man: Gen.(6) & Cxt: Trans.(H) & Man: Dir.(7) & $3 / .0033$ & $2.202++$ \\
\hline & & & & & Cxt: Trans.(H) & Cxt: Trans.(H) & Man: Dir.(7) & $3 / .0033$ & $3.426+++$ \\
\hline Spec. Ob.(T) & Pos. Fed: Spec.(2) & Pos. Fed: Spec.(2) & $6 / .0066$ & $3.407+++$ & Man: Dir.(7) & Cxt: Trans.(H) & Cxt: Trans.(H) & $3 / .0033$ & $2.291++$ \\
\hline Spec. Ob.(T) & Pos. Fed: Spec.(2) & Confus. Stat.(Y) & $3 / .0033$ & $2.581+++$ & & & & & \\
\hline VI: Pmpt.(9) & Pos. Fed: Spec. (2) & Cxt: Tchr. Per.(G) & $3 / .0033$ & $2.761+++$ & Cxt: Tchr. Cen.(F) & Spec. Ob.(T) & Pos. Fed: Spec.(2) & $5 / .0055$ & $6.780+++$ \\
\hline \multirow[t]{2}{*}{ Cxt: Tchr. Cen.(F) } & Pos. Fed: Spec. (2) & Man: Dir.(7) & $3 / .0033$ & $2.202++$ & Man: Dir.(7) & Spec. Ob.(T) & Pos. Fed: Spec.(2) & $4 / .0044$ & $3.049+++$ \\
\hline & & & & & Cxt: Tchr. Per.(G) & Spec. Ob.(T) & Cxt: Tchr. Cen.(F) & $4 / .0044$ & $4.672+++$ \\
\hline Man: Dir.(7) & Man: Dir.(7) & Man: Dir.(7) & $15 / .0165$ & $2.163++$ & Man: Dir.(7) & Spec. Ob.(T) & VI: Pmpt.(9) & $3 / .0033$ & $1.652+$ \\
\hline Cxt: Trans.(H) & Man: Dir.(7) & Cxt: Trans.(H) & $11 / .0121$ & $11.558+++$ & VI: Str./Rul.(Q) & Spec. Ob.(T) & Pos. Fed: Spec.(2) & $3 / .0033$ & $5.409+++$ \\
\hline Man: Dir.(7) & Man: Dir.(7) & Gen. Ob. (1) & $7 / .0077$ & $2.233++$ & Pos. Fed: Spec.(2) & Spec. Ob.(T) & VI: Pmpt.(9) & $3 / .0033$ & $3.772+++$ \\
\hline Gen. Ob.(1) & Man: Dir.(7) & Spec. Ob.(T) & $5 / .0055$ & $3.011+++$ & Cxt: Tchr. Per.(G) & Spec. Ob.(T) & Pos. Fed: Spec.(2) & $3 / .0033$ & $3.155+++$ \\
\hline Man: Dir.(7) & Man: Dir.(7) & Cxt: Trans.(H) & $4 / .0044$ & $1.757+$ & Man: Dir.(7) & Spec. Ob.(T) & Cxt: Tchr. Per.(G) & $3 / .0033$ & $2.090++$ \\
\hline Man: Dir.(7) & Man: Dir.(7) & Cor. Fed: Neg. B.(5) & $4 / .0044$ & $4.448+++$ & & & & & \\
\hline Man: Dir.(7) & Man: Dir.(7) & Man: Gen.(6) & $4 / .0044$ & $1.757+$ & & & & & \\
\hline Gen. Ob.(1) & Man: Dir.(7) & Cxt: Tchr. Cen.(F) & $4 / .0044$ & $2.750+++$ & & & & & \\
\hline Cxt: Tchr. Per.(G) & Man: Dir.(7) & Cxt: Tchr. Cen.(F) & $3 / .0033$ & $3.056+++$ & & & & & \\
\hline VI: Pmpt.(9) & VI: Pmpt.(9) & VI: Pmpt.(9) & $18 / .0198$ & $6.482+++$ & & & & & \\
\hline Spec. Ob.(T) & VI: Pmpt.(9) & VI: Pmpt.(9) & $6 / .0066$ & $2.506++$ & & & & & \\
\hline Spec. Ob.(T) & VI: Pmpt.(9) & Pos. Fed: Spec.(2) & $4 / .0044$ & $2.461++$ & & & & & \\
\hline
\end{tabular}




\begin{tabular}{|l|l|l|l|l|l|l|l|l|l|}
\hline Pos. Fed: Spec.(2) & VI: Pmpt.(9) & VI: Pmpt.(9) & $3 / .0033$ & $2.132++$ & & & \\
\hline Cxt: Tchr. Cen.(F) & VI: Pmpt.(9) & VI: Pmpt.(9) & $3 / .0033$ & $2.422++$ & & & \\
\hline & & & & & & & & \\
\hline Man: Dir.(7) & Cxt: Tchr. Cen.(F) & Man: Dir.(7) & $6 / .0066$ & $2.817+++$ & & & & \\
\hline Gen. Ob.(1) & Cxt: Tchr. Cen.(F) & Man: Dir.(7) & $5 / .0055$ & $2.961+++$ & & & \\
\hline VI: Pmpt.(9) & Cxt: Tchr. Cen.(F) & VI: Pmpt.(9) & $3 / .0033$ & $2.422++$ & & & \\
\hline Spec. Ob.(T) & Cxt: Tchr. Cen.(F) & VI: Pmpt.(9) & $3 / .0033$ & $1.883+$ & & & \\
\hline Spec. Ob.(T) & Cxt: Tchr. Cen.(F) & Pos. Fed: Spec.(2) & $3 / .0033$ & $3.155+++$ & & & \\
\hline
\end{tabular}

Note. $+\mathrm{p}<.05,++\mathrm{p}<. .01,+++\mathrm{p}<.001$ 
The differences regarding the type of three event sequence chains for the T-In and the TOut detail that the T-In had double the three event sequence chains with instructionally relevant fulcrums than the T-Out, with six versus three.

Regarding the amount of chains interacting with the fulcrums in each teacher's lesson, the T-Out had the most numerous precursors and successors regarding the fulcrums that occurred two or more times out of the chains in the data. The T-Out had the most numerous chains interacting with the fulcrums of Management: Direction (7) and Specific Observation (T) at nine and eight chains. However, when examining the precursors and successors regarding these fulcrums, it was evident there were differences in the types of elements that were in these chains. Regarding both of these fulcrums for the T-Out, some of the precursors and successors were managerial, contextual and instructional and non-instructional.

This evidence of conceptually different precursors and successors was not the case for the most numerous three event sequence chains for the T-In. In fact, for the T-In, the most numerous chains interacting with one fulcrum was the fulcrum of the Verbal Instruction: Prompt (9) element with six triple chains occurring in conjunction with this element. Regarding the elements that were either precursors or successors in relation to this element, each was conceptually related, so they were instructionally relevant. The elements of Positive Feedback: Specific (2), Verbal Instruction: Prompt (9) and Specific Observation (T) followed or preceded this fulcrum.

When examining the T-In three event sequence chains, other interesting differences emerged. All of the chains regarding the Management: Direction (7) element as a fulcrum exhibited either managerial, contextual precursors or successors. This details some conceptual relation. This was again different for the T-In than for the T-Out, with the array of elements that 
were precursors or successors with the same fulcrum. Other interesting differences were that the T-In exhibited the Verbal Instruction: Skill Explanation (8) element, the Verbal Instruction: Strategies/Rules (Q) element and the Modeling (W) element as fulcrums and the T-Out did not. When the T-In exhibited these three elements as fulcrums, all of the precursors and successors were instructionally relevant.

Regarding the five-second lag that was put into place for the three event sequence chains, drastic differences within teacher's data were not evident. Each teacher's data reflected changes in the chains upheld, but the lag did not bring about any substantial differences within the data or between the two teachers.

In summation. The three event sequence differences for each teacher were substantial regarding the amount of instructionally relevant fulcrums and the type of elements that were precursors or successors to these nested elements. The T-In had double the instructionally relevant elements as fulcrums when compared to the T-Out. Of the precursors and successors exhibited with these fulcrums that were instructionally relevant, three total for the T-Out, the elements were not all conceptually related. They were contextual, instructional and noninstructional for the Positive Feedback: Specific (2) element and contextual and instructional for the Verbal Instruction: Prompt (9) element and managerial, contextual, instructional for the Specific Observation (T) elements acting as fulcrums.

This was not the case for the T-In. Of the fulcrums that were instructionally relevant for the T-In, all of the precursors and successors of these six were instructionally relevant. The T-In basically had more instructionally relevant elements as fulcrums and for those fulcrums had all instructionally relevant precursors and successors. Also, even when examining the Management: 
Direction (7) element as a fulcrum, the predictability in precursors and successors for the conceptually related elements was more apparent for the T-In than the T-Out.

Sequence Differences - Students

Two event sequence. The differences between the S-In and the S-Out in the two event sequence chains are described below. Abbreviations of key words for the student's categories in the chains have been provided in Table 12.

Table 12-Student Elements Alpha-numeric Key

\begin{tabular}{|c|c|}
\hline Element & Abbreviation (Chains) \\
\hline 1. $\quad$ Motor Appropriate (behavioral element) & Mot. App.(1) \\
\hline 2. Motor Inappropriate (behavioral element) & Mot. Inapp.(2) \\
\hline 3. $\quad$ Motor Supporting (behavioral element) & Mot. Sup.(3) \\
\hline 4. $\quad$ Cognitive: Passive (behavioral element) & Cog: Pas.(4) \\
\hline 5. Cognitive: Active (behavioral element) & Cog: Act.(5) \\
\hline 6. $\quad$ On Task Management: Verbal (behavioral element) & On. Tsk. Man: Verb.(6) \\
\hline 7. $\quad$ On Task Management: Non-Verbal (behavioral element) & On. Tsk. Man: Non-Verb.(7) \\
\hline 8. $\quad$ Off Task (behavioral element) & Of. Tsk.(8) \\
\hline 9. $\quad$ Interim (behavioral element) & Intrm.(9) \\
\hline 10. (0) Waiting (behavioral element) & Wait.(0) \\
\hline 11. (A) Context: Review (contextual element) & Cxt: Rev.(A) \\
\hline 12. - & - \\
\hline 13. - & - \\
\hline 14. (D) Context: Music Off (contextual element) & Cxt: Mus. Of.(D) \\
\hline 15. (E) Negative Self-worth Comment(behavioral element) & Neg.Self-wrth.Com.(E) \\
\hline 16. (F) Context: Teacher Position Central (contextual element) & Cxt: Tchr. Cen.(F) \\
\hline 17. (G) Context: Teacher Position Periphery (contextual element) & Cxt: Tchr. Per.(G) \\
\hline 18. (H) Context: Transition (contextual element) & Cxt: Trans.(H) \\
\hline 19. - & - \\
\hline 20. (J) Context: Indecision (contextual element) & Cxt: Indec.(J) \\
\hline 21. (K) Context: Pupil Configuration Warm Up (contextual element) & Cxt: Pup. Conf. Wrm. Up.(K) \\
\hline 22. (L) Context: Pupil Configuration Center (contextual element) & Cxt: Pup. Conf. Cen.(L) \\
\hline
\end{tabular}




\begin{tabular}{|c|l|}
\hline $23 .-$ & - \\
\hline $24 . \quad-$ & - \\
\hline $25 . \quad(O)$ Context: Lesson Preview (contextual element) & Cxt: Les. Prev.(O) \\
\hline $26 . \quad$ (P) Context: Instruction (contextual element) & - \\
\hline $27 .-$ & - \\
\hline $28 . \quad-$ & Cxt: Mus. On(S) \\
\hline $29 . \quad$ (S) Context: Music On (contextual element) & - \\
\hline $30 . \quad-$ & - \\
\hline $31 . \quad-$ & - \\
\hline $32 . \quad-$ & Confus.(W) \\
\hline $33 . \quad$ (W) Confusion (behavioral element) & - \\
\hline $34 . \quad-$ & - \\
\hline $35 . \quad-$ & Cxt: Pup. Conf. Dril.(Z) \\
\hline $36 . \quad$ (Z) Context: Pupil Configuration Drillwork (contextual element) & \\
\hline
\end{tabular}

Tables 13 and 14 have delineated the chains for the students regarding the two event sequences. 
Table 13-Summary Two Event Sequence S-In

\begin{tabular}{|c|c|c|c|c|c|c|c|}
\hline Trigger & Successor & Freq./Prob. & Z-score & Trigger & Successor & Freq./Prob. & Z-score \\
\hline \multirow[t]{3}{*}{ Mot. App.(1) } & Wait.(0) & $19 / .0578$ & $4.389+++$ & Cxt: Tchr. Per.(G) & Cxt: Tchr. Cen.(F) & $7 / .0213$ & $3.606+++$ \\
\hline & Mot. Inapp.(2) & $6 / .0182$ & $3.175+++$ & & & & \\
\hline & & & & Cxt: Trans.(H) & Cxt. Tchr. Per.(G) & $5 / .0152$ & $2.804+++$ \\
\hline \multirow[t]{2}{*}{ Mot. Inapp.(2) } & Mot. App.(1) & $4 / .0122$ & $1.746+$ & & Cxt: Mus. On(S) & $3 / .0091$ & $3.527+++$ \\
\hline & Wait.(0) & $4 / .0112$ & $2.093++$ & & Cxt: Mus. Of.(D) & $3 / .0091$ & $3.527+++$ \\
\hline \multirow[t]{2}{*}{ Cog. Pas.(4) } & Cog. Act.(5) & $7 / .0213$ & $3.691+++$ & Cxt. Mus. On(S) & Cxt: Tchr. Cen.(F) & $3 / .0091$ & $3.019+++$ \\
\hline & Of. Tsk.(8) & $4 / .0122$ & $2.944+++$ & & & & \\
\hline Cog. Act.(5) & Cog. Pas.(4) & $12 / .0365$ & $7.616+++$ & & & & \\
\hline On. Tsk. Man: Non-Verb.(7) & Wait.(0) & $9 / .0274$ & $1.656+++$ & & & & \\
\hline Of. Tsk.(8) & Cog. Pas.(4) & $3 / .0091$ & $2.045+++$ & & & & \\
\hline \multirow[t]{2}{*}{ Wait.(0) } & Mot. App.(1) & $17 / .0517$ & $3.574+++$ & & & & \\
\hline & Mot. Inapp.(2) & $4 / .0122$ & $1.971++$ & & & & \\
\hline Cxt: Mus. Of.(D) & On. Tsk. Man: Non-Verb.(7) & $3 / .0091$ & $2.296++$ & & & & \\
\hline & & & & & & & \\
\hline
\end{tabular}




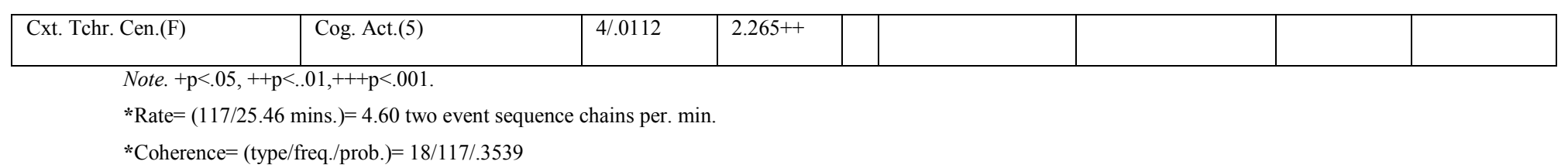

Table 14-Summary Two Event Sequence S-Out

\begin{tabular}{|c|c|c|c|}
\hline Trigger & Successor & Freq./Prob. & Z-score \\
\hline Mot. App.(1) & Mot. Inapp.(2) & $3 / .0080$ & $2.474+++$ \\
\hline Mot. Inapp.(2) & Cxt. Tchr. Per.(G) & $6 / .0160$ & $2.170++$ \\
\hline & Intrm.(9) & $3 / .0080$ & $1.739+$ \\
\hline Mot. Sup.(3) & Of. Tsk.(8) & $3 / .0080$ & $3.003+++$ \\
\hline & Cxt. Tchr. Per.(G) & $3 / .0080$ & $1.659+$ \\
\hline Cog. Pas.(4) & Mot. App.(1) & $5 / .0133$ & $3.555+++$ \\
\hline On. Tsk. Man: Non-Verb.(7) & Cxt: Trans.(H) & $5 / .0133$ & $2.690+++$ \\
\hline & Cxt: Indec.(J) & $3 / .0080$ & $3.687+++$ \\
\hline Of. Tsk.(8) & Wait.(0) & $8 / .0213$ & $2.976+++$ \\
\hline Intrm.(9) & Mot. Inapp.(2) & $4 / .0107$ & $2.660+++$ \\
\hline Wait.(0) & Cxt. Tchr. Per.(G) & $13 / .0347$ & $3.175+++$ \\
\hline
\end{tabular}




\begin{tabular}{|l|l|l|l|}
\hline & & & \\
\hline Cxt: Tchr. Cen.(F) & Of. Tsk.(8) & $12 / .0320$ & $3.367+++$ \\
\hline & Intrm.(9) & $9 / .0240$ & $2.516++$ \\
\hline Cxt: Tchr. Per.(G) & & & \\
\hline & Cxt: Tchr. Cen.(F) & $25 / .0667$ & $5.517+++$ \\
\hline Cxt: Trans.(H) & & & \\
\hline & Cxt. Trans.(H) & $12 / .0320$ & $5.075+++$ \\
\hline & On. Tsk. Man: Non-Verb.(7) & $5 / .0113$ & $2.625+++$ \\
\hline Cxt. Indec.(J) & & & \\
\hline \multicolumn{2}{|l|}{ Note. $+\mathrm{p}<05,++\mathrm{p}<.01,+++\mathrm{p}<001.008$} & $2.247++$ \\
\hline
\end{tabular}

Note. $+\mathrm{p}<.05,++\mathrm{p}<. .01,+++\mathrm{p}<.001$.

$*$ Rate $=(122 / 27.8$ minutes $)=4.39$ two event sequence chains per. min.

${ }^{*}$ Coherence $=($ type $/$ freq $/$ prob. $)=17 / 122 / .3233$ 
Regarding the S-In data, the Motor Appropriate (1) element occurred in 4 out of the 18 two event sequence chains. When examining this element further, three out of the five clusters of two event sequence chains that occurred for the S-In had the Motor Appropriate (1) element in these chains as either a trigger or successor.

For the S-In, the Cognitive: Passive (4) element occurred four times in these chains as either a trigger or a successor and the Cognitive: Active (5) element occurred three times in this same manner. Consequently, when examining the Motor Appropriate (1), the Cognitive: Passive (4), and the Cognitive: Active (5) elements, all three combined acted as a trigger or successor in 9 out of the 18 two event sequence chains. Each of these three elements provided a content learning function (see Appendix B for definitions). Therefore, content learning elements occurred in 9 out of the 18 two event sequence chains for the S-In.

Another interesting observation when examining the S-In, was that four out of five clusters of two event sequence chains included the content learning elements previously mentioned in at least one of two chains in each cluster. These occurred as either a trigger or successor in these clusters of two event sequence chains. Regarding the S-Out data, only one out of five clusters of two event sequence chains included a content learning element as a trigger. This element was the Motor Supporting (3) element and it did not show up in the S-In chains. The S-In also exhibited a two event sequence chain that had the trigger of the Motor Inappropriate (2) element and the successor of the Motor Appropriate (1) element. Basically, this showed a non-content learning element as a trigger and then a content learning element following. This type of chain was not evident for the S-Out. 
When examining the S-Out, the S-Out exhibited the Motor Appropriate (1) element in 2 of the 17 two event sequences. The S-Out did not exhibit this element as a trigger with more than one successor, or in a cluster, as the S-In indicated. The S-Out also exhibited this Motor Appropriate (1) element in two less chains than the S-In. The element of Cognitive: Passive (4) occurred once for the S-Out and the Cognitive: Active (5) element did not occur in the S-Out data. This differs from the S-In, in that these two elements described above occurred in a combined total of five chains for the S-In. Therefore, when examining the content learning elements in these chains, the S-In exhibited content learning elements 11 times and in 9 of the 18 chains, while the S-Out exhibited these content learning elements 5 times and in 4 of the 17 chains.

The Motor Supporting (3) element occurred in 2 out of the 17 two event sequence chains for the S-Out and occurred as a trigger both times. The successors that followed were the Off Task (8) and Context: Teacher Position Periphery (G) elements.

When examining the Interim (9) element, it occurred for the S-Out as a trigger and occurred three times total, as a trigger or successor. This element did not occur for the S-In. When the Off Task (8) element occurred for the S-Out, it occurred in chains with the Waiting (0), Context: Teacher Position Central (F), and Motor Supporting (3) elements. With the S-In this element occurred with the Cognitive: Passive (4) element both times. Another interesting difference between the two teachers was related to the Waiting (0) element. The S-In exhibited this in five times in the data as a trigger or successor, while the S-Out exhibited it twice.

In summation. The type of two event sequence chains for the students with the most successors or clusters that followed one trigger was different for the S-In and the S-Out. The S-In exhibited more chains with content learning elements. These included more chains with the 
triggers of the Motor Appropriate (1), Cognitive: Passive (4), and Cognitive: Active (5)

elements. The S-In also exhibited more of these content learning elements in the clusters than the S-Out. Out of the four clusters consisting of a trigger and two successors that followed, the S-In exhibited content learning elements in at least one of the two chains, citing content learning involvement in each of these four clusters. This was not the case for the S-Out, who only had one cluster out of the five clusters exhibited that included a content learning element, the Motor Supporting (3) element. Regarding the Off Task (8) behavior, the S-Out exhibited one more chain with this element as a trigger or successor and for the Waiting (0) element, the S-In exhibited this as a trigger or successor three more times.

The five second lag that was put into place contributed to interesting decreases within the student's data. Substantially fewer chains were upheld for the S-In when the lag was introduced. However, the most numerous chains that were upheld for the S-In included content learning elements. The most numerous chains that were upheld for the S-Out included non-content learning elements. These chains included ones with the Off Task (8) and Motor Inappropriate (2) elements. Furthermore, the T-Out chains did not change as substantially when the lag was introduced.

Three event sequence. The differences between the S-In and the S-Out in the three event sequence chains are described below. Abbreviations of key words for the student's categories in the chains have been provided in Table 12. Tables 15 and 16 have delineated the chains for each teacher regarding the three event sequences. 
Table 15-Summary Three Event Sequence S-In

\begin{tabular}{|c|c|c|c|c|}
\hline Precursor & Fulcrum & Successor & Freq./Prob. & Z-score \\
\hline Wait.(0) & Mot. App.(1) & Wait.(0) & $9 / .0274$ & $4.544+++$ \\
\hline Wait.(0) & Mot. App.(1) & Mot. Inapp.(2) & $4 / .0122$ & $4.621+++$ \\
\hline Mot. Inapp.(2) & Mot. App.(1) & Wait.(0) & $3 / .0091$ & $3.463+++$ \\
\hline Cog. Pas.(4) & Mot. App.(1) & Mot. App.(1) & $3 / .0091$ & $2.179++$ \\
\hline Mot. App.(1) & Mot. Inapp.(2) & Mot. App.(1) & $4 / .0122$ & $3.272+++$ \\
\hline Wait.(0) & Mot. Inapp.(2) & Wait.(0) & $3 / .0091$ & $3.463+++$ \\
\hline Cog. Pas.(4) & Cog. Act.(5) & Cog. Pas.(4) & $7 / .0213$ & $7.309+++$ \\
\hline Cxt: Tchr. Cen.(F) & Cog. Act.(5) & Cog. Pas.(4) & $3 / .0091$ & $3.963+++$ \\
\hline Mot. App.(1) & Wait.(0) & Mot. App.(1) & $13 / .0396$ & $6.027+++$ \\
\hline Mot. App.(1) & Wait.(0) & Mot. Inapp.(2) & $4 / .0122$ & $4.285+++$ \\
\hline On. Tsk. Man: Non-Verb.(7) & Wait.(0) & Cog. Pas.(4) & $4 / .0122$ & $3.080+++$ \\
\hline Mot. Inapp.(2) & Wait.(0) & Mot. App.(1) & $3 / .0091$ & $3.117+++$ \\
\hline
\end{tabular}

Note. $+\mathrm{p}<.05,++\mathrm{p}<. .01,+++\mathrm{p}<.001$

Table 16 Summary Three Event Sequence S-Out

\begin{tabular}{|l|l|l|l|l|}
\hline Precursor & Fulcrum & Successor & Freq./Prob. & Z-score \\
\hline Cxt: Tchr. Cen.(F) & Of. Tsk.(8) & Wait.(0) & $3 / .0080$ & $1.753+$ \\
\hline Cxt: Tchr. Cen.(F) & Of. Tsk.(8) & Of. Tsk.(8) & $3 / .0080$ & $2.100++$ \\
\hline Cog. Pas.(4) & Of. Tsk.(8) & Cog. Pas.(4) & $3 / .0080$ & $5.351+++$ \\
\hline Of. Tsk.(8) & Of. Tsk.(8) & Wait.(0) & $3 / .0080$ & $3.753+++$ \\
\hline & & & & \\
\hline Cxt: Tchr. Per.(G) & Wait.(0) & Cxt: Tchr. Cen.(F) & $6 / .0160$ & $4.649+++$ \\
\hline Of. Tsk.(8) & Wait.(0) & Cxt: Tchr. Per.(G) & $5 / .0134$ & $3.580+++$ \\
\hline Cxt: Tchr. Cen.(F) & Wait.(0) & Cxt: Tchr. Per.(G) & $3 / .0080$ & $3.213+++$ \\
\hline Cxt: Trans.(H) & Wait.(0) & Cxt: Trans.(H) & $3 / .0080$ & $3.888+++$ \\
\hline & & & & \\
\hline Cxt: Tchr. Per.(G) & Cxt: Tchr. Cen.(F) & Cxt: Tchr. Per.(G) & $8 / .0214$ & $2.174++$ \\
\hline Cxt: Tchr. Per.(G) & Cxt: Tchr. Cen. (F) & Of. Tsk.(8) & $7 / .0187$ & $3.575+++$ \\
\hline
\end{tabular}




\begin{tabular}{|l|l|l|l|l|}
\hline & & & & \\
\hline Wait.(0) & Cxt. Tchr. Per.(G) & Cxt: Tchr. Cen.(F) & $8 / .0214$ & $4.585+++$ \\
\hline Cxt: Tchr. Cen.(F) & Cxt. Tchr. Per.(G) & Cxt: Tchr. Cen.(F) & $6 / .0160$ & $3.301+++$ \\
\hline Intrm.(9) & Cxt. Tchr. Per.(G) & Cxt: Tchr. Cen.(F) & $4 / .0107$ & $3.462+++$ \\
\hline Of. Tsk.(8) & Cxt. Tchr. Per.(G) & Of. Tsk.(8) & $3 / .0080$ & $4.839+++$ \\
\hline & & & & \\
\hline On. Tsk. Man: Non-Verb.(7) & Cxt: Trans.(H) & Cxt: Trans.(H) & $3 / .0080$ & $3.888+++$ \\
\hline Cxt: Trans.(H) & Cxt: Trans.(H) & Cxt: Trans.(H) & $3 / .0080$ & $1.860+$ \\
\hline \multicolumn{1}{|l|}{ Note. $+\mathrm{p}<.05,++\mathrm{p}<. .01,+++\mathrm{p}<.001$} & & \\
\hline
\end{tabular}

The most noticeable difference between the S-In and the S-Out was the amount of fulcrums that were content learning elements. The S-In exhibited content learning elements in two of the four, three event sequences where the fulcrums had more than one chain in relation to it. These elements that acted as fulcrums were the Motor Appropriate (1) and Cognitive: Passive (5) elements.

When examining the S-Out, the S-Out did not exhibit one content learning element as a fulcrum in any of the five, three event sequence elements that occurred more than once regarding a fulcrum. Furthermore, the fulcrum of the Off Task (8) element was exhibited in the S-Out three event sequence chains. This element also occurred in conjunction with other three event sequences that occurred with other fulcrums, which had more than one corresponding precursor and successor in relation to it. This means that the Off Task (8) element occurred in conjunction with three out of the five fulcrums which met the previous criteria for inclusion. This Off Task (8) element was not exhibited in the S-In chains in any form.

When examining the chains of the S-In, there were two chains out of all the ones involved with the fulcrums that included all content learning elements as a series of three, citing a precursor, fulcrum and successor relationship. These were the Cognitive: Passive (4), the Motor Appropriate (1) and the Motor Appropriate (1) elements in a series and the Cognitive: 
Passive (9), Cognitive: Active (5) and Cognitive: Passive (4) elements in a series. The S-Out did not exhibit any content learning elements in a series of three elements in conjunction, and as stated previously, did not have a fulcrum that was a content learning element either.

In summation. The differences between the S-In and S-Out were substantial regarding the three event sequence chains. Half of the fulcrums exhibited for the S-In were content learning elements. The S-In also exhibited a series of three elements that were all content learning elements in a row: precursor, fulcrum, and successor, for two chains regarding the four fulcrums exhibited.

The S-Out did not exhibit any fulcrums that were content learning elements. Furthermore, the S-Out exhibited the Off Task (8) element as a fulcrum and the T-In did not exhibit this element as a fulcrum, or as a precursor or successor in any of the series of two chains or more relating to any fulcrum. Finally, out of the five fulcrums that occurred with chains of two or more in the data, three of the five fulcrums had chains with the Off Task (8) element.

Regarding the five-second lag that was put into place for the three event sequence chains, drastic differences within teacher's data were not evident. Each teacher's data reflected changes in the chains upheld, but the lag did not bring about any substantial differences within the data or between the two teachers. The elements that remained after the lags paralleled the type of chains that occurred prior to them, just in less number.

\section{Graphical Data}

Teachers. The differences between the T-In and the T-Out regarding the graphical data are described below. Graphs 1 and 2 have provided a visual representation of the elements throughout time for each teacher. 


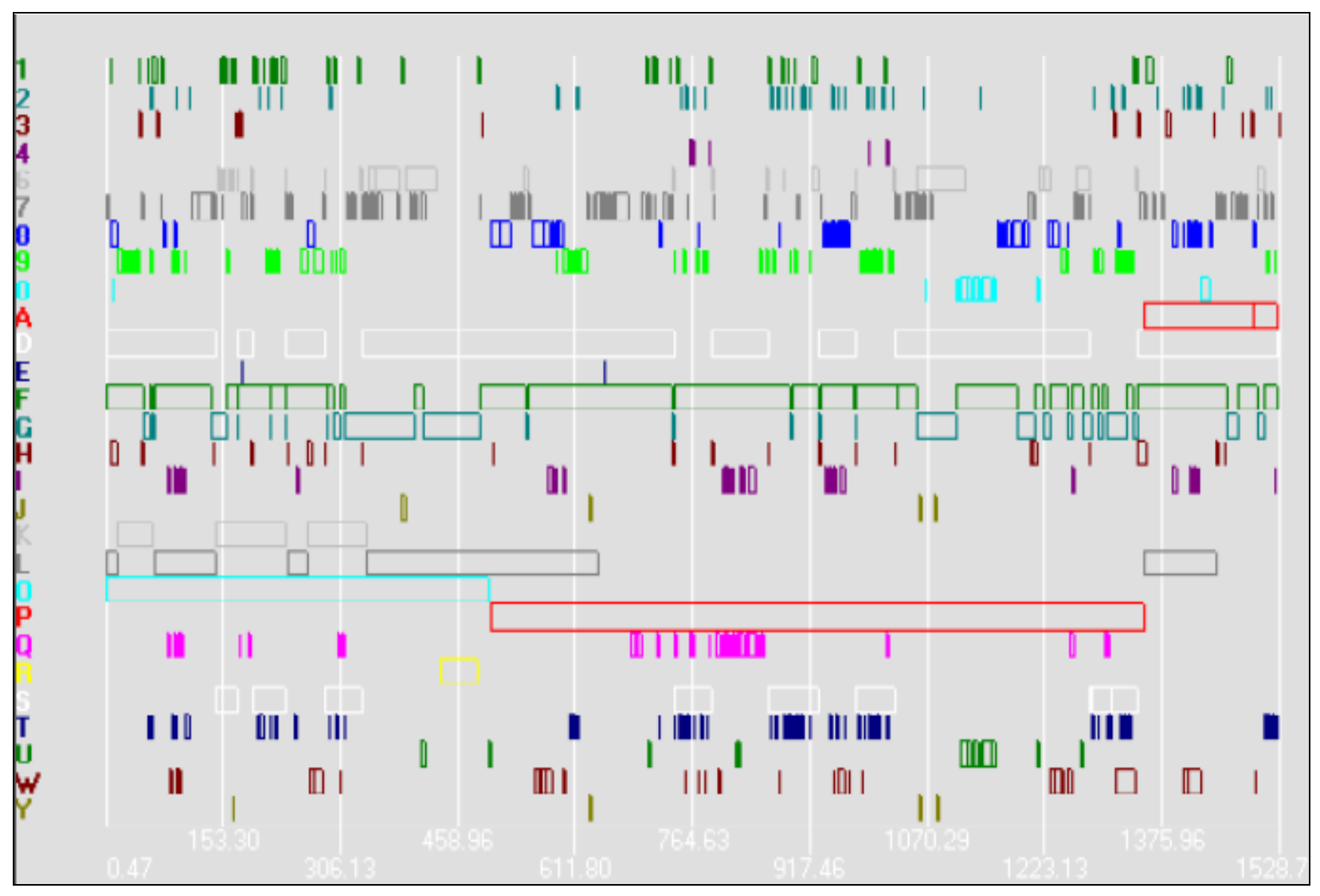

Graph 1: T- In Data 


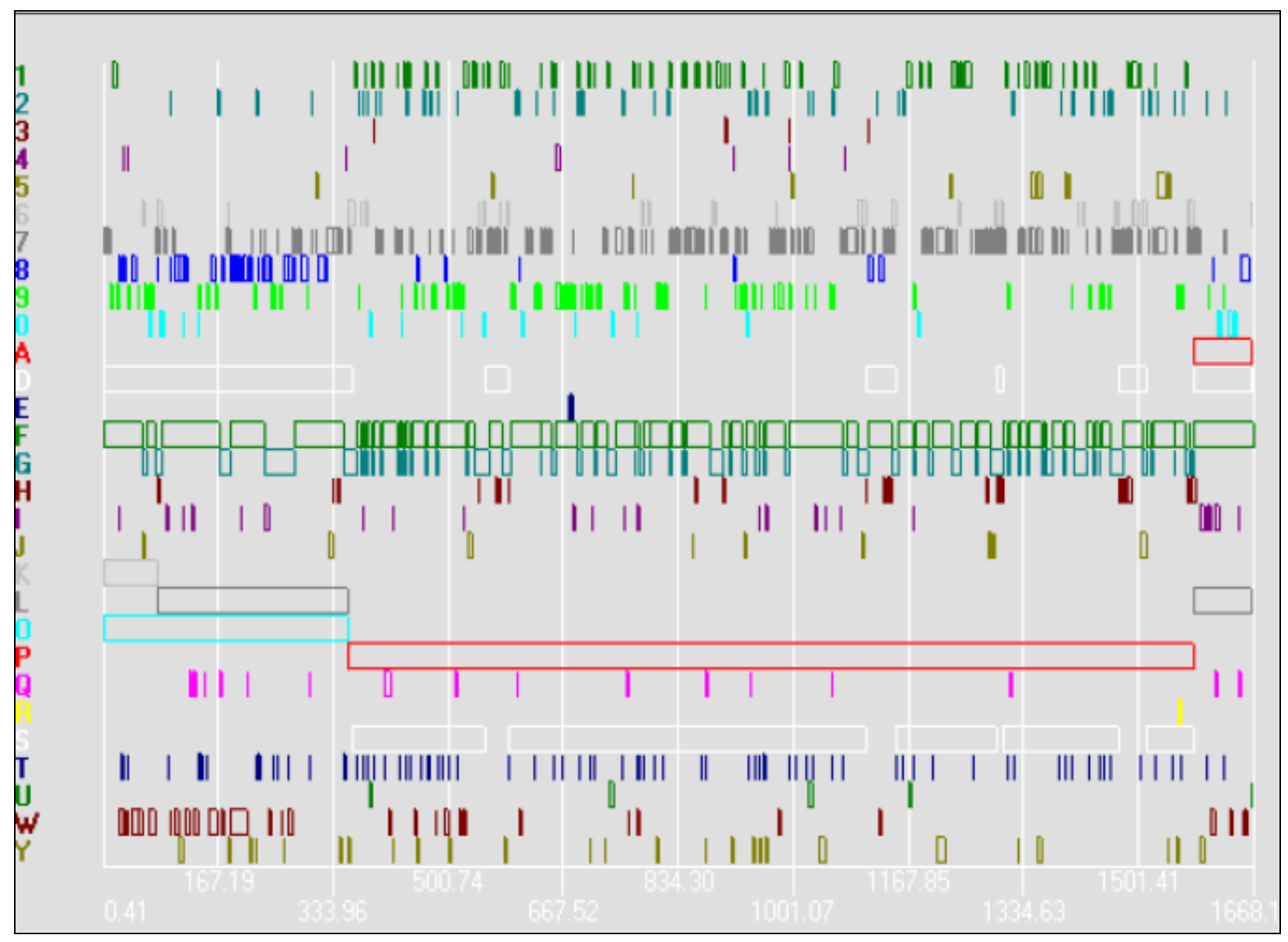

Graph 2: T-Out Data 
When examining the teacher data, there were substantial differences between the frequency, duration and clusters of elements regarding how and when they occurred temporally. Regarding the General Observation (1) element, the T-In exhibited this element throughout the lesson in the Context: Lesson Preview, the Context: Instruction (P) and the Context: Review (A) element portions. The T-In exhibited this element with fewer occurrences and in a more spread out pattern than the T-Out. The T-Out differed regarding this element in that the T-Out exhibited this element more frequently and primarily in the Context: Instruction $(\mathrm{P})$ element portion of the lesson.

The Positive Feedback: General (3) element was exhibited throughout the lesson in all portions of the lesson for the T-In and the T-Out. The difference was that the T-In exhibited this element substantially more in frequency, in duration and in more clusters than the T-Out. The TOut exhibited this element in a more singular fashion.

The Corrective Feedback: Skill (4) element detailed differences for the T-Out and the TIn. The T-Out exhibited this element in the Context: Lesson Preview $(\mathrm{O})$ and the Context: Instruction $(\mathrm{P})$ portions of the lesson and at a higher frequency than the T-In. The T-In only exhibited this in the Context: Instruction (P) element portion of the lesson and exhibited less frequency, duration and clusters of this element. The T-In had more singular events of this element and the T-Out had more clusters. Regarding the Corrective Feedback: Negative Behavior (5) element, the T-Out exhibited this element in more frequency, duration and clusters than the T-In. The T-Out exhibited this element in the Context: Lesson Preview (A) element portion and the Context: Instruction (P) element portion of the lesson. The T-In did not exhibit this element in any way. 
The management elements differed in a temporal pattern when the T-In and T-Out were examined. The Management: General (6) element occurred in longer duration for the T-In than the T-Out as the equipment was set up during the T-In lesson as opposed to the T-Out setting up the equipment beforehand. This element usually began when the Context: Music Off (D) element began. This setting up equipment by the T-In and the lack thereof by the T-Out led to shorter durations of this elements occurrence for the T-Out. Both teachers exhibited this element throughout the entire lesson. However, when examining the Management: Direction (7) element, the T-Out exhibited this element at a higher frequency than the T-In. Basically, for the T-Out, if the Management: Direction (7) element occurred, another Management: Direction (7) element was soon to follow. Both teachers exhibited these two management elements similarly in regards to when it happened throughout the lesson.

When examining the verbal instruction elements the differences were vast between the two teachers. Regarding the Verbal Instruction: Skill Explanation (8) element, the T-In exhibited this element in more frequency, in longer duration and in more clusters throughout the lesson than the T-Out. The T-In exhibited this element primarily in the Context: Instruction $(\mathrm{P})$ and Context: Review (A) portions of the lesson. The T-Out exhibited this element in clusters primarily in the Context: Lesson Preview (O) portion of the lesson. The T-Out exhibited this element in other portions as well such as the Context: Instruction (P) element and Context: Review (A) element portions, but in far less frequency than the T-In.

The Verbal Instruction: Prompt (9) element also differed substantially for each of the two teachers. The T-In exhibited more clusters of this element and the T-Out exhibited more isolated incidents of it throughout time. When this element was exhibited, another element of the same 
type followed with more predictability for the T-In. Both teachers exhibited this element similarly in regards to when it occurred in the lesson.

The Verbal Instruction: Relating Statement (0) element detailed differences between the two teachers as well. The T-Out exhibited this element while in the Context: Teacher Position Periphery (G) element and the Context: Teacher Central (F) element and the T-In only exhibited this element in the Context: Teacher Position Central (F) element. Furthermore, when this element was exhibited by each teacher, the T-In exhibited this element in longer duration each time. However, the T-Out exhibited this element more often and in smaller clusters, spread out in the opening, middle and closure, while the T-In exhibited it in the latter two parts of the lesson and in larger clusters.

Regarding the last verbal instruction element, the Verbal Instruction: Strategies/Rules (Q) element, the T-In exhibited more clusters of this element than the T-Out and in longer duration each time it was exhibited. The T-In exhibited most of these clusters in the Context: Instruction (P) element portion of the lesson, while the T-Out exhibited it throughout all of the portions of the lesson in more spread out, small cluster exhibitions.

When examining the Teacher Questioning: Subject Matter (I) element, the T-In exhibited more clusters and in longer duration for each occurrence of this element within these clusters. The T-In exhibited this element only when the Context: Music Off (D) element occurred and sometimes began soon after this music stopped. The T-Out exhibited this element with both the Context: Music Off and Context: Music On categories. The T-Out also exhibited this element in more spread out, less complex clusters.

Regarding the elements of Context: Teacher Position Central (F) and Context: Teacher Position Periphery (G) there was a substantial difference in the amount of time spent for each 
teacher regarding each element. During the Context: Instruction $(\mathrm{P})$ element, the T-In spent far greater time in the Context: Teacher Position Central (F) element than the T-Out. Furthermore, the T-Out went back and forth between the central and periphery positioning much more quickly during the Context: Instruction (P) element.

Regarding the Context: Indecision $(\mathrm{J})$ element, the T-Out exhibited a higher frequency of occurrence for this element regarding the clusters. This occurred during both the Content: Music On (S) and Context: Music Off (D) elements. This element only occurred in clusters during the Context: Music Off (D) element for the T-In.

Another element that exhibited visual differences for the T-Out and the T-In was Specific Observation (T). The T-In exhibited larger clusters for this element than the T-Out. The T-In exhibited this element most often in clusters as soon as the Context: Music On (D) element was present. The T-Out exhibited this element throughout the lesson, but in more isolated incidents and smaller clusters. The T-Out exhibited this when the Context: Music On (D) element was present, but there was not an immediacy of occurrence such as the T-In data reflected.

The two teachers differed in the Teacher Answers (U) element as well regarding the visual data. In almost every instance of this element, the T-Out first exhibited the Teacher Questioning: Subject Matter (I) element just prior to this element of Teacher Answers (U). It is almost as if the question that was asked by the T-Out was answered by the T-Out, or that the TOut reiterated the answer. The T-Out also exhibited this element in less frequency, in less duration and in less clusters than the T-In. The T-In exhibited this element in a cluster only once after the Teacher Questioning: Subject Matter (I), suggesting that the T-In students had a firmer grasp on the material. The answering of questions by the T-In occurred more often, were longer in duration and were in larger, more numerous clusters. 
Regarding the Modeling (W) element, the T-In exhibited this element throughout all portions of the lesson and the T-Out primarily exhibited it in the Context: Lesson Preview (O) element portion of the lesson. Basically, the T-Out modeled how content should be done before it was done in the lesson by the students, but revisited it far less than the T-In throughout the lesson.

When examining the Confusing Statement (Y) element, the T-Out exhibited this element in more frequency, duration and in more clusters throughout all portions of the lesson. The T-Out exhibited this directly before and after the Modeling (W) element throughout the lesson, which created a situation where the T-Out gave a statement that was confusing directly before or after the content was modeled by this teacher.

In summation. Notable differences between the two teachers were exhibited when looking at how and when the data appeared visually in the graphs. The most obvious differences in the data were that the T-In exhibited more tightly packaged clusters of elements for the Verbal Instruction: Prompt (9), the Verbal Instruction: Strategies/Rules (Q), the Teacher Questioning: Subject Matter (I), Specific Observation (T) and Teacher Answers (U) elements. The T-In exhibited elements which occurred when other contextual elements began or ended in a more predictable pattern, such as in conjunction with the Context: Music On (S) or Context: Music Off (D) elements and the Context: Instruction (P) elements. Also, the T-In exhibited more instructional elements throughout the entire lesson than the T-Out. The T-Out exhibited a less packaged more spread out element pattern that lacked temporal contiguity with other elements. The T-Out exhibited elements with less of a pattern or predictability regarding the visual data in the graphs and only exhibited some of the instructional elements in certain portions of the lesson. 
The T-Out also exhibited the Management: Direction (7) element far more than the T-In throughout the entire lesson with less predictability as to how or when it would occur.

\section{Graphical Data}

Students. The differences between the S-In and the S-Out regarding the graphical data are described below. Graphs 3 and 4 have provided a visual representation of the elements throughout time for the student's data.

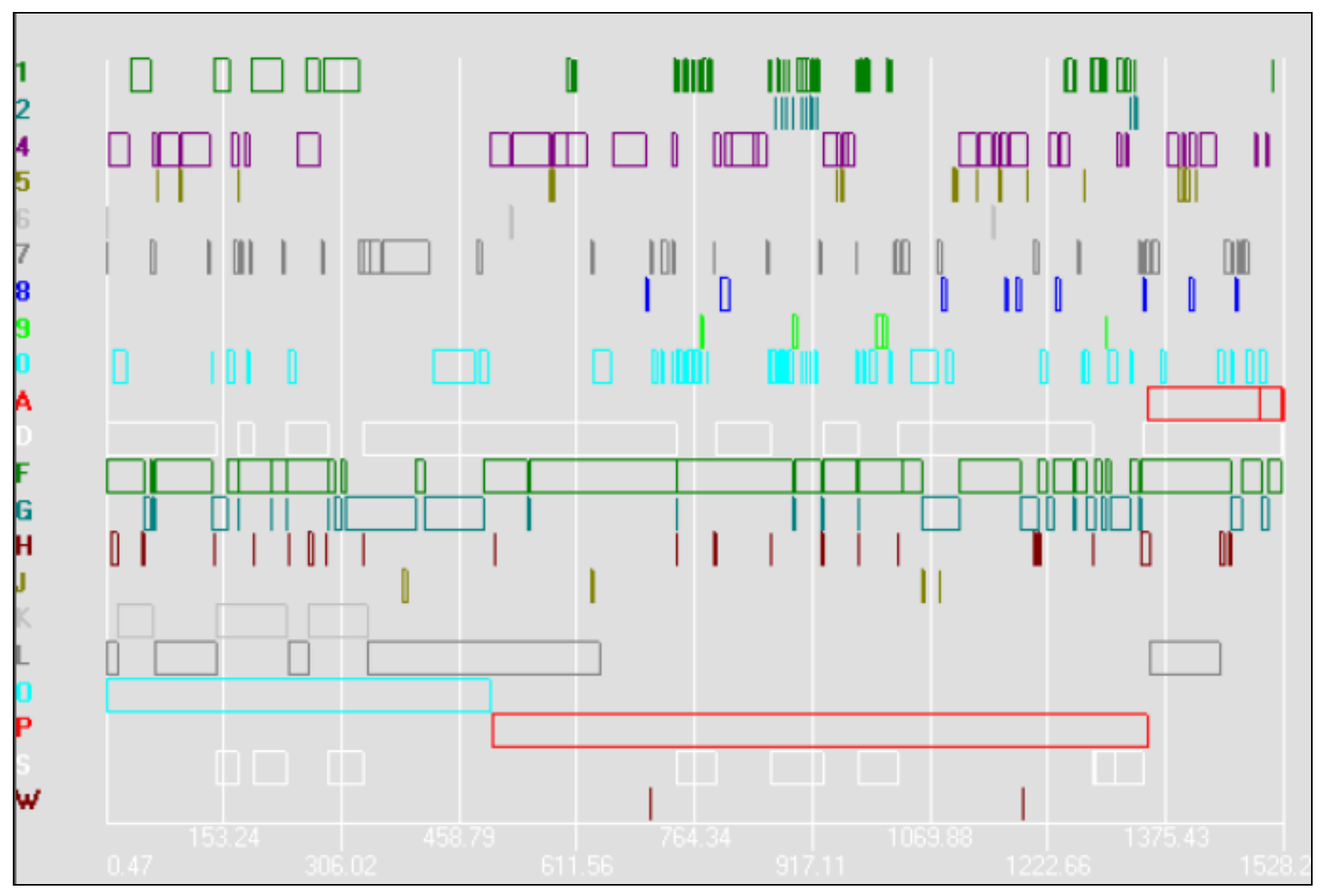

Graph 3: S-In Data 


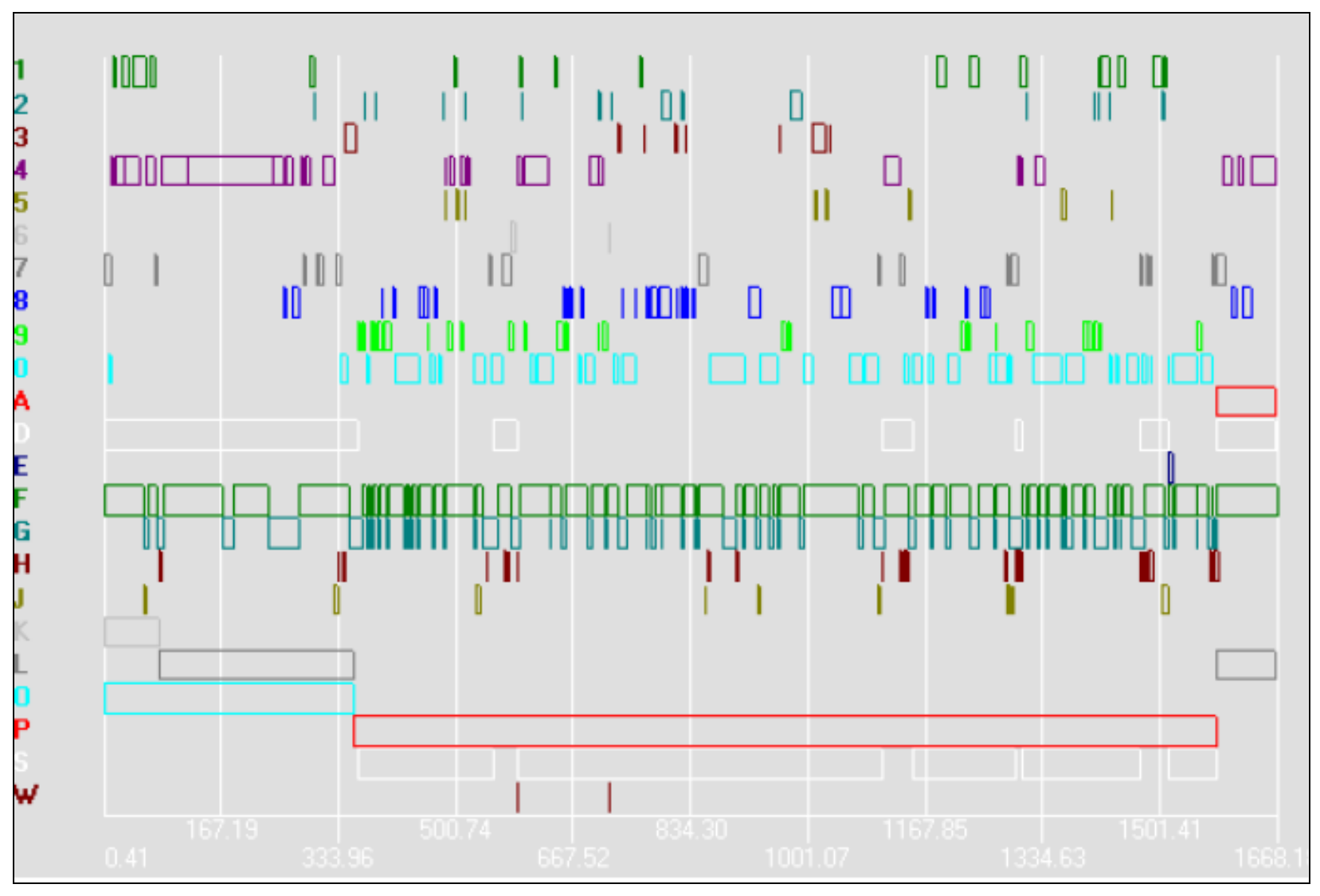

Graph 4: S-Out Data 
When examining the student's data, there were substantial differences between the frequency, duration and clusters of elements regarding how and when they occurred temporally. Regarding the Motor Appropriate (1) element, the S-In exhibited this element in more frequency, duration and clusters than the S-Out. The S-In had more of a temporal pattern regarding this element as it occurred in more clusters and most often directly before, after or even during the Cognitive: Passive (4) element. The most clusters regarding the Motor Appropriate (1) element came during the Context: Instruction (P) element portion of the lesson for the S-In. Regarding the Motor Appropriate (1) element for the S-Out, this element did not often precede or follow the Cognitive: Passive (4) element in the data and only occurred simultaneously with this element in the beginning of the lesson. This element for the S-Out was spread throughout the lesson with less of a pattern and in smaller clusters.

Regarding the Motor Inappropriate (2) element, the S-Out exhibited this element more frequently and in longer duration throughout all portions of the lesson. The S-Out exhibited these elements in a more spread out manner and with less of a pattern than the S-In. When the S-In exhibited this element it was in a more clustered format and occurred more closely in time before or after the Motor Appropriate (1) and Waiting (0) elements. The Motor Inappropriate (2) element only occurred in the Context: Instruction (P) element portion of the lesson for the S-In, and when it occurred, it occurred in shorter duration when compared to the S-Out.

The Motor Supporting (3) element only occurred for the S-Out and it occurred during the Context: Instruction (P) element portion of the lesson. The element also occurred most frequently during the Context: Teacher Position Periphery $(\mathrm{G})$ element.

The Cognitive: Passive (4) element was exhibited by the S-In most often during the Context: Music Off (D) element and in all of the portions of the lesson, which are again the 
Context: Lesson Preview (O), the Context: Instruction (P) and the Context: Review (A) elements. Furthermore, for the S-In, the Motor Appropriate (1) element usually preceded, followed or occurred simultaneously with this element, detailing a temporal relationship with other elements. The S-Out exhibited this element with substantially less frequency, duration and clusters. This element in the S-Out lesson did not exhibit the same temporal pattern with other elements as the S-In lesson exhibited. This element occurred most often in the Context: Lesson Preview $(\mathrm{O})$ element portion of the lesson for the S-Out. After this exhibition in the beginning of the lesson, this element occurred in a sparse manner throughout the rest of the lesson when compared to the S-In.

Regarding the Cognitive: Active (5) element, the S-In exhibited this element in the preview, instruction and review portions of the lesson. This element was exhibited with more frequency, in more clusters and with a more visible temporal relationship with the Cognitive: Passive (4) element for the S-In than the S-Out. The Cognitive: Active (5) element only occurred in the Context: Instruction (P) element portion of the lesson for the S-Out, and occurred with less frequency in conjunction with the Cognitive: Passive (4) element and other conceptually related elements.

When examining the On Task Management: Non-Verbal (7) element, the S-In exhibited this element in more frequency, duration and clusters. This element was exhibited throughout all portions of the lesson and occurred most often directly before or after the Waiting $(0)$ element in the lesson. The S-Out exhibited this element far less than the S-In.

Regarding the Off Task (8) element, the S-Out exhibited this element substantially more in frequency, duration and clusters than the S-In. This element occurred often times during the Context: Teacher Position Central (F) element for the S-Out. This element occurred in all of the 
portions of the lesson for the S-Out including the preview, instruction and review. This was not the case for the S-In, for when this element did occur, it occurred in the latter two contextual portions of the lesson.

When examining the Waiting (0) element, the S-In exhibited this element in greater frequency regarding the clusters, but in less duration for each singular occurrence. This element occurs throughout the entire lesson for the S-In, but occurred most often in the Context: Instruction $(\mathrm{P})$ element portion for the S-Out.

Regarding the Off Task (8) and Waiting (0) elements for the S-In and the S-Out, interesting differences pertaining to when these elements occurred in relation with other elements was visibly evident. These two elements, especially the Waiting (0) element usually occurred directly before or after the On Task Management: Non-Verbal (7) element for the S-In and occurred throughout the entire lesson. For the S-Out these two elements occurred most often during the instructional portion of the lesson. The exhibitions of the Waiting (0) element were much longer for the S-Out than for the S-In regarding each exhibition and the Off Task (8) element occurred far more often for the S-Out. These two elements usually occurred in relation to the Motor Appropriate (1), Motor Inappropriate (2) and Motor Supporting (3) elements from a temporal standpoint for the S-Out.

In summation. Notable differences between the student's data were exhibited when looking at how and when the data appeared visually in the graphs. The S-In data exhibited more elements that were in more tightly packed clusters, which occurred in time very closely to other conceptually related elements. For example, the S-In exhibited more clusters of elements regarding the Motor Appropriate (1) element, while occurring in time closely if not during the Cognitive: Passive (4) element. This was not the case for the S-Out data, which exhibited more 
spread out elements that were smaller in clusters and which did not always immediately precede or succeed another relevant element. The elements that did often times occur closely in time before or after the content learning elements for the S-Out were the Off Task (8) and Waiting (0) elements. Similarly, regarding the S-Out, often times even when the Context: Teacher Position Central (F) element was exhibited, the Off Task (8) element was evident. In conclusion, the S-In exhibited more complexity regarding the elements in clusters and also was more predictable as to when these clusters would occur in the data when compared to the S-Out.

\section{Data Interpretation}

The five dimensions of field systems mentioned earlier have been used to understand the data exhibited in the systems under analysis. This dimensional analysis has been based on the Sharpe and Hawkins (1992b) study. These dimensions have focused primarily on the temporal and contextual relationships that have been revealed in the data analysis.

1. Frequency and duration: Each individual element has been analyzed to determine the number and length of occurrence represented in the systems. The substantial teacher system differences between these two teachers regarding these two dimensions were that the T-In exhibited more instructional elements and in longer duration than the T-Out. An example of this for the T-In was regarding the element of Verbal Instruction: Skill Explanation (8), where the TIn exhibited this element more frequently and in longer duration. Other elements, such as the Context: Teacher Position Central (F) element was substantially longer in duration when exhibited by the T-In versus the T-Out. Furthermore, the T-Out exhibited far more occurrences of the Management: Direction (7) element than the T-In. Therefore, when examining each teacher's behavioral systems regarding the instructional elements, the T-In exhibited a higher frequency and duration for these specific elements. 
In examining the student system differences between the S-In and the S-Out regarding frequency and duration of elements, substantial differences again emerged. Regarding the content learning elements, the S-In exhibited these elements with more frequently and in longer duration than the S-Out. An example of this was the Motor Appropriate (1) element, which was exhibited by the S-In more frequently and in longer duration each time it was exhibited. The SOut exhibited a behavioral system which reflected more non-content learning elements than the S-In. An example of this was the Motor Inappropriate (2) element, which was exhibited by the SOut in substantially more frequency and duration.

2. Rhythm: This term describes the temporal patterns of elements, chains, or clusters of elements or occurrences of patterns throughout the instructional episode. Frequency and relative probability of temporal patterns have been analyzed relative to tempo, regularity and complexity of elements throughout time.

The substantial differences between these two teachers regarding tempo was that when examining the elements in each teacher's system, the T-In exhibited elements in conjunction with other elements far more quickly once a trigger was exhibited in a chain. A temporal pattern, or tightly packed elements, exhibiting less time after a trigger in the two event sequence data and less time after a precursor or less time before a successor regarding the three event sequence data were exhibited by this T-In. An example of how the T-In exhibited a behavioral system which reflected greater tempo in elements exhibited was for the Verbal Instruction: Prompt (9) element. When this element was exhibited as a trigger regarding the cluster, successors in the two event sequence chains, and precursor and successors in the three event chains regarding the fulcrum occurred substantially more quickly in conjunction with this element and others in the TIn behavioral system. Regarding the tempo of the elements exhibited, the T-Out behavioral 
system exhibited substantially less of a tempo with triggers and successors in the two event sequence data, or precursors, fulcrums and successors in conjunction with each other in the three event sequence data. More time elapsed before another element was exhibited in conjunction to a trigger or fulcrum throughout all of the data for the T-Out. Consequently, the data reflected less tempo in the T-Out behavioral system.

Regarding the regularity of data exhibited, the T-In exhibited substantially more chains in more tightly packed clusters once a trigger occurred, and especially for the instructionally related elements. The same evidence was exhibited amongst the three event sequence data. The T-Out element data were more sparsely exhibited for the majority of the elements and especially the instructional elements and was less predictable in regards to elements in conjunction with each other. An example of this was for the element of Teacher Questioning: Subject Matter (I), where the T-In exhibited this in more clusters throughout the lesson. Once again, the most substantial differences were regarding the instructional element differences between the two teachers.

When examining the complexity of the data exhibited, the T-In exhibited more complexity in the clusters than the T-Out. Regarding the majority of the elements, the clusters were more complex in that more elements occurred more closely in succession and often times occurred simultaneously with other elements for the T-In. An example of this was the Verbal Instruction: Skill Explanation (8) element that occurred in more complex clusters and in conjunction with the Teacher Questioning: Subject Matter (I) element.

When examining the tempo of elements in the student system for the S-In and S-Out, substantial differences were evident. The S-In exhibited elements much more quickly related to the triggers and the clusters were more tightly packed. Basically, for an element that occurred, another element was soon to follow regarding the trigger. This was also evident for the three 
event sequence data. An example of this was with the Motor Appropriate (1) element, which not only occurred more frequently for the S-In, but when it occurred elements closely followed that were of the same element. Therefore, specifically examining of the content learning elements, the S-In exhibited the majority of these behavioral elements at a quicker tempo when a trigger or fulcrum of these elements were exhibited.

Regarding the regularity of the student data, the S-In exhibited substantially more chains in more tightly packed clusters once a trigger occurred. This was especially evident for the content learning elements. More clusters of the Motor Appropriate (1), the Cognitive: Passive (4), and the Cognitive: Active (5) elements were present for the S-In. The S-Out exhibited more of the Motor Supporting (3) element as this element was not present in the S-In data. Therefore, when examining the two systems as a whole, regarding the content learning elements the S-In exhibited more regularity.

When examining the complexity of the elements in the student behavioral systems for the S-In and S-Out, differences again emerged. The S-In exhibited more complex clusters than the SOut. This was the most evident regarding the content learning elements. Basically, if a trigger or fulcrum occurred in the data for the S-In, other elements were soon to follow or occurred simultaneously with these elements. Even though the complexity was more evident for the S-In, the apparent predictability was more evident as well. An example of this was that the Motor Appropriate (1) element clusters occurred in more tightly packed clusters in the data. Furthermore, this Motor Appropriate (1) element was in close conjunction temporally with the Waiting (0) element. This complexity was not as evident for the S-Out, as the data were more sparsely exhibited in singular elements and in clusters. 
3. Complexity: This variable defines the system based on the number of individual categories that have been used to describe the stimulus and behavioral elements in the instructional episodes. This complexity has been determined by the number of categories created. Regarding the complexity of the data, the T-Out represented a more complex behavioral system. This was due to the fact that the T-Out exhibited more categories of behavioral elements than the T-In. When the type of behavioral elements was examined, however, the T-Out exhibited only one more category than the T-In. Furthermore, this category exhibited was the Corrective Feedback: Negative Behavior (5) element. Basically, the T-Out exhibited a more complex system, but it pertained to the T-Out exhibiting a non-instructional element.

When examining the S-In and the S-Out, the S-Out exhibited a more complex behavioral system. This was due to the fact that the S-Out had two more behavioral categories exhibited in the data set. These categories were the Motor Supporting (3) and the Negative Self-worth Comment (E) elements. One of these elements was a content learning element and the other was a non-content learning element. Therefore, more types of elements were exhibited in the S-Out system.

4. Coherence: This category described to what degree each of the elements was consistently related to each other. Coherence represents the degree or order and consistency in a setting. The BEST software has determined these probabilities. High probabilities of high frequency within a few dual chains reflect a greater organizational coherence or predictability. Low probabilities of low frequency within many dual chains indicate less coherence.

The T-In exhibited higher probabilities of higher frequency amongst fewer types of two event sequence chains than the T-Out. This was regarding the inclusion criteria of the chain occurring more than three or more times in the data and at a minimum statistical significance of 
.05 . The T-In exhibited 37 types of two event sequence chains, at a frequency of 372 chains and at a total probability of .5104 , while the T-Out exhibited 40 types of two event sequence chains, at a frequency of 367 chains and at a total probability of .4037 . Although these differences were not drastic, the T-In exhibited a more coherent system. Furthermore, when examining the types of two event sequence chains, the T-In exhibited more conceptually related elements within those chains than the T-Out.

The data regarding the coherence were very similar for the S-In and the S-Out. The S-In exhibited 18 types of two event sequence chains, at a frequency of 117 chains and at a total probability of .3539 , while the S-Out exhibited 17 types of two event sequence chains, at a frequency of 122 chains and at a total probability of .3233. Although these differences were not drastic, and the T-In represented higher probabilities of relatively the same frequency and same types of elements in the data, the type of chains exhibited once again painted a clearer picture as to what elements in the student's data were more coherent. The S-In exhibited more coherence toward content learning elements than the S-Out. For example, in the clusters of two chains, one trigger and two different successors for each teacher, the S-In exhibited content learning elements in all four of the clusters, while the S-Out only exhibited one content learning cluster out of the five.

5. Velocity: The frequency of occurrence for each element and sequence pairs has been analyzed to determine differences in elemental velocity. Differences in elemental velocities regarding both singular and sequence pairs have been summarized.

The differences in the descriptive data, the data describing how the singular elements were exhibited in the two teacher's lessons were evident. The T-Out exhibited a higher velocity of elements that occurred singularly in the data. The rate or velocity of these elements was 32.80 
elements per minute for the T-Out. The T-In exhibited 28.75 elements per minute for the elements that occurred singularly in the data. Therefore, the T-Out exhibited a higher velocity of elements occurring per minute than the T-In. However, when examining which elements occurred with the highest unconditional probability in each teacher's data, differences were again substantial. The elements with the highest unconditional probability for the T-Out were first the Management: Direction (7) element and second the Verbal Instruction: Prompt (9) element. This Management: Direction (7) element occurred almost twice as much for the T-Out than for the TIn. The elements with the highest unconditional probability for the T-In were first the Verbal Instruction: Prompt (9) element and second the Management: Direction (7) element. The T-In also exhibited the Verbal Instruction: Prompt (9) element more often than the T-Out. The T-In also exhibited all of the instructional elements in the system more often when combined than the T-Out.

When examining the velocity or rate for the two event sequence chains for the T-In, the T-In exhibited more chains per minute than the T-Out. The T-In exhibited 14.61 chains per minute, while the T-Out exhibited 13.20 chains per minute. Not only did the T-In exhibit a higher rate of elemental change per minute, but there were more instructional elements exhibited for the T-In than for the T-Out. Therefore, the T-Out exhibited a higher velocity of singular elements occurring in the system, but when looking at predictability of these elements from a chain standpoint, the rate of elements was more predictable for the instructional elements in sequence for the T-In.

When examining the velocity or rate for the S-In and the S-Out differences again emerged. Regarding the descriptive data or elements that occurred singularly, the S-Out exhibited a higher rate of change than that S-In. The rate for the S-Out was 13.56 elements per 
minute, while the rate for the S-In was 13.04 elements per minute. However, when examining the type of elements occurring for the students, differences were again substantial. The elements with the highest unconditional probability from a behavioral standpoint for the S-Out were first the Waiting (0) element and second the Off Task (8) element. The elements with the highest unconditional probability for the S-In were first the Motor Appropriate (1) element followed by the Waiting (0) element. The S-In exhibited this Motor Appropriate (1) element substantially more than the S-Out, but the Waiting (0) element occurred more for the S-In as well. The Off Task (8) element occurred substantially less for the S-In. From a standpoint of content learning elements, with elements that occurred singularly in the data, the S-In exhibited more of these elements in combined total than the S-Out. Therefore, the velocity was higher for the S-Out, but this pertained to all of the elements in the system and not specifically to the content learning elements.

When examining the velocity or rate for the two event sequence chains for the S-In and the S-Out, the S-In exhibited more chains per minute than the S-Out. The S-In exhibited 4.60 chains per minute, while the S-Out exhibited 4.39 chains per minute. Once again, as the previous dimensional data delineated, the content learning elements were more prevalent in the chains exhibited for the S-In. Furthermore, this paralleled the singular element data, in that more content learning elements were exhibited.

\section{Interview Data}

The auditory interviews with questions and answers for each teacher have been summarized for the T-In (Appendix C) and the T-Out (Appendix D). The differences between the answers of each teacher regarding the standardized interview questions are summarized below. (See Table 17). 
Table 17-Interview Answers Summarized

\begin{tabular}{|c|c|c|}
\hline Categories of Answers & Teacher's Answers Regarding Questions & Participant and location in text \\
\hline \multicolumn{3}{|l|}{ Type of Master's Education } \\
\hline & $\begin{array}{l}\text { "Master's administration, ah, educational } \\
\text { leadership" }\end{array}$ & T-Out, Line 5 \\
\hline & $\begin{array}{l}\text { "Master's degree in physical education } \\
\text { teacher education" }\end{array}$ & T-In, Line 5 \\
\hline \multicolumn{3}{|l|}{$\begin{array}{l}\text { Relationship of Master's Degree and } \\
\text { Behavioral Outcome }\end{array}$} \\
\hline & $\begin{array}{l}\text { "Educational leadership it allowed me to be } \\
\text { able to help the students" }\end{array}$ & T-Out, Line 9, 10 \\
\hline & "Delegation of roles" & T-Out, Line 10 \\
\hline & "Area of focus in behavior, um, management" & T-Out, Line 10,11 \\
\hline & "Helps with many students that are off task" & T-Out, Line 11 \\
\hline & $\begin{array}{l}\text { "Needs a little bit of extra, um, } \\
\text { encouragement" }\end{array}$ & T-Out, Line 11,12 \\
\hline & "With different, ah, behaviors" & T-Out, Line 12 \\
\hline & "Discipline situations that arise" & T-Out, Line 12, 13 \\
\hline & $\begin{array}{l}\text { "It helped me refresh myself on a lot of things } \\
\text { I had as an undergrad" }\end{array}$ & T-In, Line 10, 11 \\
\hline & "It helped" & T-In, Line 11 \\
\hline & "By getting the master's degree it helped" & T-In, Line 13 \\
\hline & "It helped me refocus" & T-In, Line 13, 14 \\
\hline & $\begin{array}{l}\text { "The little things that you learn about, but you } \\
\text { never really get to apply" }\end{array}$ & T-In, Line 14,15 \\
\hline & $\begin{array}{l}\text { "Little things they get lost in the shuffle of } \\
\text { being a first year teacher, so it helped" }\end{array}$ & T-In, Line 15,16 \\
\hline & "It helped me refresh on those things" & T-In, Line 16 \\
\hline & "You know, refine my teaching" & T-In, Line 17 \\
\hline Why Specific Master's Education & & \\
\hline
\end{tabular}




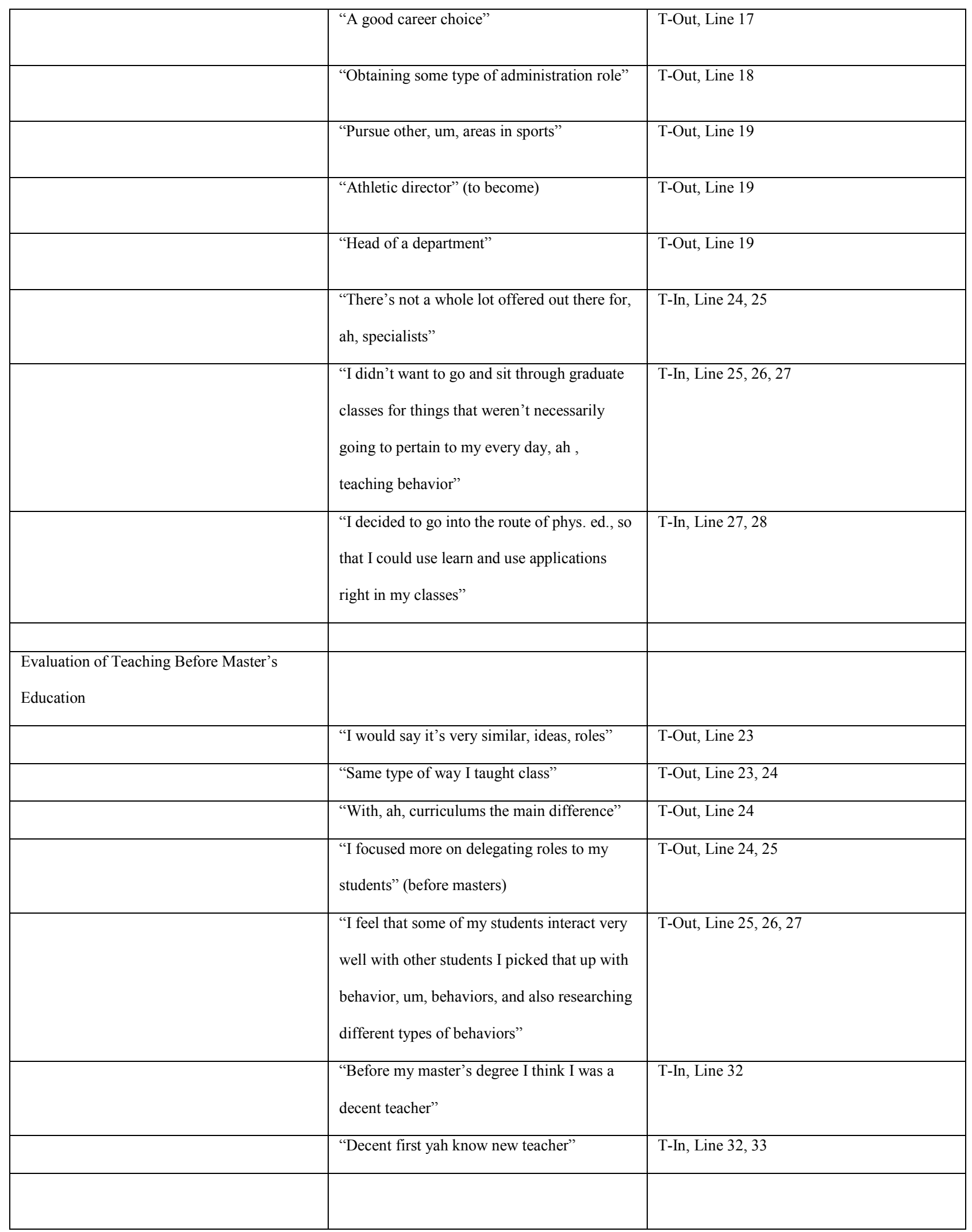




\begin{tabular}{|c|c|c|}
\hline $\begin{array}{l}\text { Evaluation of Teaching After Master's } \\
\text { Education }\end{array}$ & & \\
\hline & "I feel it's helped" & T-Out, Line 31 \\
\hline & "I feel it's helped significantly" & T-Out, Line 31 \\
\hline & $\begin{array}{l}\text { "Just also going through the process of getting } \\
\text { my masters" }\end{array}$ & T-Out, Line 31,32 \\
\hline & "I'm happy with the decisions I did" & T-Out, Line 33 \\
\hline & "It helped me in my class work" & T-Out, Line 33 \\
\hline & $\begin{array}{l}\text { "I feel a positive manner towards my } \\
\text { students" }\end{array}$ & T-Out, Line 33, 34 \\
\hline & $\begin{array}{l}\text { "I'm molding them into a better, ah, } \\
\text { productive student" }\end{array}$ & T-Out, Line 34,35 \\
\hline & $\begin{array}{l}\text { "The master's program in phys. ed. helped me } \\
\text { realize some things that I should have been } \\
\text { doing and wasn't doing" }\end{array}$ & T-In, Line 33, 34 \\
\hline & $\begin{array}{l}\text { "It helped me, um, refine my skills a little bit } \\
\text { better" }\end{array}$ & T-In, Line 34,35 \\
\hline & $\begin{array}{l}\text { "Gave me some practical application to use in } \\
\text { different situations" }\end{array}$ & T-In, Line 35,36 \\
\hline & $\begin{array}{l}\text { "It helped in my delivery of physical } \\
\text { education, um, information and content" }\end{array}$ & T-In, Line 36,37 \\
\hline & "It's much better" (my teaching) & T-In, Line 41 \\
\hline & $\begin{array}{l}\text { "The master's program it helped me realize } \\
\text { some things" }\end{array}$ & T-In, Line 41, 42 \\
\hline \multirow[t]{5}{*}{$\begin{array}{l}\text { Role of Other Factors Alongside Master's } \\
\text { Education }\end{array}$} & & \\
\hline & $\begin{array}{l}\text { "Helped my students learn better" (masters } \\
\text { and experience) }\end{array}$ & T-In, Line 44 \\
\hline & $\begin{array}{l}\text { "Helped them understand things better" } \\
\text { (master's and experience) }\end{array}$ & T-In, Line 45 \\
\hline & $\begin{array}{l}\text { "It's been very rewarding for both myself and } \\
\text { the students I teach" (masters and experience) }\end{array}$ & T-In, Line 45,46 \\
\hline & "But also just experience alone" & T-In, Line 42 \\
\hline
\end{tabular}




\begin{tabular}{|c|c|c|}
\hline & "Experience is very valuable" & T-In, Line 43 \\
\hline & $\begin{array}{l}\text { "That experience in conjunction with the } \\
\text { master's degree in phys. ed. has really just } \\
\text { helped my teaching" }\end{array}$ & T-In, Line 43, 44 \\
\hline \multicolumn{3}{|l|}{$\begin{array}{l}\text { Coursework in Master's Education Pertaining } \\
\text { to Behavior }\end{array}$} \\
\hline & $\begin{array}{l}\text { "A few (courses) more like focusing on } \\
\text { behavior" }\end{array}$ & T-Out, Line 39 \\
\hline & "All types of behaviors" & T-Out, Line 41,42 \\
\hline & "Students that were attention seeking" & T-Out, Line 42, 43 \\
\hline & (students who were) "Off task" & T-Out, Line 43 \\
\hline & $\begin{array}{l}\text { "Some (behaviors) that I wasn't really aware } \\
\text { of" }\end{array}$ & T-Out, Line 43 \\
\hline & $\begin{array}{l}\text { "More behavior, discipline to the higher end, } \\
\text { they were physically" (unclear) }\end{array}$ & T-Out, Line 43, 44 \\
\hline & $\begin{array}{l}\text { "I wasn't sure what to do with them and now, } \\
\text { um, found out more" (unclear) }\end{array}$ & T-Out, Line 44,45 \\
\hline & "Application classes" & T-In, Line 52 \\
\hline & "Behavior" (classes) & T-In, Line 52 \\
\hline & "Behavioral science type courses" & T-In, Line 53 \\
\hline & "Fitness education courses" & T-In, Line 53 \\
\hline & $\begin{array}{l}\text { "How to best deliver fitness education } \\
\text { concepts" }\end{array}$ & T-In, Line 53, 54 \\
\hline & $\begin{array}{l}\text { "Classes dealing with special education } \\
\text { students, how to best approach teaching } \\
\text { them" }\end{array}$ & T-In, Line 54,55 \\
\hline & $\begin{array}{l}\text { "A whole, a whole gambit of things (in } \\
\text { coursework) }\end{array}$ & T-In, Line 55,56 \\
\hline & $\begin{array}{l}\text { "Teaching is dealing with behavior, so you } \\
\text { know anything that had to deal with that" }\end{array}$ & T-In, Line 56,57 \\
\hline & $\begin{array}{l}\text { "Teaching has to do with behavior of the } \\
\text { student and behavior of yourself teaching } \\
\text { those students, so you know many of the }\end{array}$ & T-In, Line $57,58,59$ \\
\hline
\end{tabular}




\begin{tabular}{|c|c|c|}
\hline & $\begin{array}{l}\text { classes, although informative also came back } \\
\text { to the behavioral end of things" }\end{array}$ & \\
\hline & $\begin{array}{l}\text { "And, ah, tied everything together" (the } \\
\text { classes) }\end{array}$ & T-In, Line 59, 60 \\
\hline \multicolumn{3}{|l|}{ Reason for Master's Education } \\
\hline & "Professional advancement" & T-Out, Line 48 \\
\hline & "Professional advancement was the main one & T-Out, Line 49 \\
\hline & "Ah I had to continue with my certification" & T-Out, Line 49,50 \\
\hline & "Self improvement" & T-In, Line 66 \\
\hline & "Professional advancement" & T-In, Line 66 \\
\hline & $\begin{array}{l}\text { "The more education you have the more } \\
\text { money you get paid" }\end{array}$ & T-In, Line 67, 68 \\
\hline & $\begin{array}{l}\text { "Knocking out two birds with one stone" (self } \\
\text { improvement and professional advancement) }\end{array}$ & T-In, Line 68,69 \\
\hline & "Trying to become a better teacher" & T-In, Line 69 \\
\hline & $\begin{array}{l}\text { "Trying to earn some extra money at the same } \\
\text { time" }\end{array}$ & T-In, Line 69, 70 \\
\hline & "Move across the pay scale" & T-In, Line 70 \\
\hline & $\begin{array}{l}\text { "Both, both of those in one were the main } \\
\text { motivation for obtaining my master's degree" }\end{array}$ & T-In, Line 70, 71 \\
\hline
\end{tabular}

Differences between the two teacher's answers or statements regarding the interview questions have been described based on answer categories. In length and detail alone, the answers for the T-In were much longer and described in more depth. The T-In also exhibited answers regarding other factors which aided in his teaching.

Regarding the category of answers created for the type of master's education, the T-Out stated, "master's administration, ah, educational leadership" (Line 5), while the T-In stated, "master's degree in physical education teacher education" (Line 5). 
Pertaining to the category of answers created for, "relationship of master's degree and behavioral outcome," each teacher described differences in what behaviors were learned regarding in their ongoing education. Examples the T-Out cited were: "behavior, um, management" (Line 10, 11), "students who are off task" (Line 11), and "discipline situations that arise" (Line 12,13) as being behaviors learned with this type of education. Examples the T-In cited were: "refreshed myself on a lot of things I had as an undergrad" (Line 10, 11), "helped me refocus" (Line 13, 14), and "refined my teaching" (Line 17). The T-Out answers reflected managerial behaviors, while the T-In answers reflected a wide variety of behaviors revisited from the physical education teacher education undergraduate education.

When examining the category of answers created for "why specific ongoing education," differences again emerged. Examples the T-Out cited were: "obtaining some type of administration role" (Line 18), "pursue other areas in sports" (Line 19), and "athletic director" (Line 19). Examples the T-In cited were: “I didn't want to go and sit through graduate classes for things that weren't necessarily going to pertain to my every day, ah, teaching behavior"' (Line 25, $26,27)$, and "I decided to go the route of phys. ed., so that I could learn and use applications right in my classes" (Line 27, 28). Once again differences between the answers were eminent. Regarding the category of answers created for “evaluation of teaching before master's education" the teacher's differed in response. Examples the T-Out cited were that his teaching was "very similar" (Line 23) and that it was the "same type of way I taught class" (Line 23, 24) before the master's degree. Examples the T-In cited were: "I think I was a decent teacher" (Line 32), and "decent first yah know new teacher" (Line 32, 33). Therefore, the T-Out cited he taught generally the same way before the master's degree as after, but the T-In cited that he was a decent teacher, but this being for a first year teacher. 
When examining the category of answers created for "evaluation of teaching after master's education" the responses were again different. Examples the T-Out cited were, "I feel it's helped" (Line 31), "just also going through the process of getting my masters" (Line 31, 32), "I feel in a positive manner towards my students" (Line 33, 34), and "I'm molding them into a better, ah, productive student" (Line 34, 35). Examples the T-In cited were: "the master's program in phys. ed. helped me realize some things that I should have been doing and wasn't doing” (Line 33, 34), “it helped me, um, refine my skills a little bit better” (Line 34, 35), “gave me some practical application" (Line 35, 36), and "it helped my delivery of physical education, um, information and content" (Line 36, 37).

Regarding the category of answers created for "coursework in master's education pertaining to behavior," each teacher's responses again differed regarding their answers. Examples the T-Out cited were: "all types of behaviors" (Line 41, 42), "off task" (Line 43), and "more behavior, discipline to the higher end, they were physically" (unclear) (Line 43, 44). Examples the T-In cited were: "behavior science classes" (Line 53), "fitness education courses" (Line 53), "how to best deliver fitness education concepts" (Line 53, 54), and "classes dealing with physical education students, how to best approach teaching them" (Line 54, 55).

When examining the category of answers created for "reason for master's education", some of the answers were similar and some divergent. Examples the T-Out cited were: "professional advancement" (Line 48) and "had to continue with my certification" (Line 49, 50). Examples the T-In cited were: "self-improvement" (Line 66), "professional advancement" (Line 66), "trying to become a better teacher" (Line 69), and "trying to earn some extra money at the same time" (Line 69, 70). 
The T-In also answered questions based on the category of answers created for "role of other factors alongside master's education" that contributed to his teaching. Examples the T-In cited were: "helped my students learn better" (masters and experience) (Line 44), "helped them understand things better" (masters and experience) (Line 45), and "that experience in conjunction with the master's degree in phys. ed. has really just helped my teaching” (Line 43, 44).

\section{Descriptive, Sequence and Interview Data}

In describing each part of the data, similarities regarding the elemental summaries, and answers each teacher provided have been disseminated. The T-In exhibited data that were more instructional and talked about it this way in his answers. The T-Out exhibited data that were more managerial and in his interview answers he spoke in this manner as well.

Regarding the T-In, more singular elements in the data and more elements in the two and three event sequence chains included instructional elements. Examples including the most complex chains, the three event sequence chains for the T-In provided a stark contrast between the T-In and T-Out data. Specific evidence regarding the three event sequence chains, or high frequency fulcrums, detailed double the instructional elements. The T-In exhibited the Verbal Instruction: Skill Explanation (8) element, the Verbal Instruction: Strategies/Rules (Q) element and the Modeling (8) elements as fulcrums or high frequency elements, and the T-Out did not.

When examining the way the T-In answered regarding the master's degree categories of answers that were created, the T-In provided statements regarding the behaviors learned from it, evaluation of teaching after it, and coursework learned in it. Examples of these were "refresh myself on a lot of things I had as an undergrad" (Line 10,11), "practical application" (Line 35, 36), "delivery of physical education, um, information and content" (Line 36, 37) and "fitness 
education concepts" (Line 53, 54). Therefore, the way the T-In talked about his ongoing education paralleled his behavioral system regarding the instructional elements taught and content elements exhibited by his students.

The T-Out data exhibited substantially more managerial elements and non-instructional elements in the singular data and in the two and three event sequences. Examples of this were how in the descriptive data alone, the T-Out exhibited approximately double the Management: Direction (7) elements. In the two event sequences the T-Out exhibited the Corrective Feedback: Negative Behavior (5) element in conjunction with the Management: Direction (7) element. This element was not exhibited in the T-In data, was non-instructional and went beyond the basic managerial tasks of the teacher. This element was exhibited by definition in relation towards students who were off task and were disobeying class rules. The three event sequence chains exhibited the same trend as the most numerous chains had the Management: Direction (7) element as the fulcrum.

Interestingly enough, when examining the way the T-Out discussed his ongoing education, the answers given reflected the singular and sequence data. When examining the categories of answers created regarding “master's degree and behavioral outcome," examples the T-Out detailed were "behavioral, um, management" (Line 10, 11), and "helps with many students that are off task" (Line 11). Another answer given for the T-Out regarding the question of "coursework in master's education pertaining to behavior," was "more behavior discipline to the higher end" (Line 43, 44). Therefore, the way the T-Out talked about his ongoing education paralleled his behavioral system regarding the managerial elements taught and non-content elements exhibited by his students. 


\section{CHAPTER FIVE}

\section{Discussion}

The findings in this case study suggested that discipline-specific ongoing education may play a role in developing expertise in physical education. The relationship of the results found in this study, along with previous research in other fields examining how expertise can possibly be gained through ongoing education, and the analysis of it through FSA in this instance may bring about insight into the continuing development of physical education teachers.

\section{A Behavioral Explanation}

Developing expertise through experience has been a topic of interest within the educational community and so a specific research study in physical education has analyzed these subjects in conjunction (Sharpe \& Hawkins, 1992b). However, analyzing the role ongoing education may play in this development has been a neglected area of analysis. In attempting to analyze expertise, FSA is a method that can be used to address this void. Though we are unable to generalize the results of this novel study to other situations, invaluable information has been gained through this FSA regarding the role ongoing education may play in expertise development.

Teachers. When the T-In and the T-Out elemental and interview answer data were analyzed, substantial differences emerged. The T-In was more instructionally focused regarding the behavioral system data and in the way that he talked about his teaching. The T-In behavioral system also reflected more expertise based on more of a temporal relationship existing between the elements. The T-Out was less instructionally focused and more managerially focused regarding the behavioral system data and in the way he talked about his teaching. Furthermore, 
the T-Out behavioral system reflected less expertise because less of a temporal relationship existed between the elements.

When examining time alone, differences in how the teachers spent their time in their lessons was evident. The T-In exhibited longer durations of the Context: Lesson Preview (O) and Context: Lesson Review (A) elements than the T-Out. The Context: Pupil Configuration Warm Up (K) element was much longer for the T-In, and this teacher also brought his students back into the center of the gym more often to revisit the content. This was evident for the T-In, as he used more Verbal Instruction: Skill Explanation (8), Teacher Questioning: Subject Matter (I) and Teacher Answers (U) elements in his lesson. These were interesting differences because the TOut exhibited the Context: Lesson Preview (O) element for a shorter amount of time than for the T-In, then exhibited the Context: Pupil Configuration Drillwork (Z) element for a longer period of time than for the T-In. The Context: Music On (S) element was exhibited in much longer duration when present in the lesson for the T-Out. The stations would change and frequently the music was left on, as the T-Out would talk over the stereo, stating where the students should go next. The T-In, on the other hand, had the Context: Music Off (D) element exhibited more often and did not try to talk over the music or the students and kept control through more frequent inquiry and positive feedback. The T-In exhibited a more positive atmosphere by providing substantially more Positive Feedback: General (3) elements directed towards the students. The research study by Sharpe and Hawkins (1992b) has reinforced this notion of encouragement and positive reinforcement being used in a more effective manner over high frequency managerial and corrective episodes.

When the Specific Observation (T) element was exhibited by the T-Out in the sequences, some of the elements exhibited in conjunction with this element were of the managerial type. 
The T-Out also used more Corrective Feedback: Skill (4) elements to adjust the students' behavior and less verbal instruction elements in combined total. The T-Out also exhibited the behavior of looking at his watch throughout the lesson, but the T-In did not exhibit this behavior. Regarding the T-In, when he walked around during the lesson, the Management: General (6) element was exhibited more often because he was picking up and setting up equipment for the next activity. The T-Out lesson was set up beforehand, as the stations were created before the class started. The T-In on the other hand set up the facility during the lessons. This was a very interesting difference because based on the instructional design of the lesson, the T-In spent more time setting up during the actual lesson, which was reflected by the Management: General (6) element percentages.

The T-Out also exhibited the Corrective Feedback Negative Behavior (5) element in his lesson and this element was not exhibited in the T-In lesson. This evidence of more negative behavior by the T-Out seemed to reflect the statement of Sharpe and Hawkins (1992b), which revealed the need for more behavioral control due to the lack of positive and instructional behavior. The T-Out was less positive, more managerial, and less enthusiastic regarding the introduction and closure of the lesson, based on the elements exhibited. More confusing statements, fewer answered questions, and shorter explanations of what students were asked to do were reflected by this teacher's data.

When examining the instructional and managerial elements, the T-In was consistently involved with more complex yet probable instructional elements, while the T-Out exhibited more managerial elements. The T-In exhibited a higher velocity of elemental chains that were not only more instructional, but were also more predicable when examining the elements that were in conjunction with these instructional behaviors. It could again be argued that less management 
was needed in the T-In lesson because more questioning and revisiting of content occurred in the T-In lesson. An environment that is more positive, which was reflected in the T-In lesson, while exhibiting instructional elements that are of high-velocity, has been stated by other researchers as being a necessity for effectiveness (Cruickshank, 1976; Goldberger, 1984; Graham \& Heimerer, 1981; McAvoy, Hursh, Nardi, Savage \& Walls, 1988; Sharpe \& Hawkins, 1992b; Thaxton, Rothstein, \& Thaxton, 1977; Walls, Nardi, von Minden \& Hoffman; 2002).

Regarding teacher positioning, there were substantial differences between the T-In and the T-Out. The T-In exhibited far longer central positioning than the T-Out. Furthermore, when the T-In exhibited this Context: Teacher Position Central (F) element, it was for a much longer duration. The T-Out exhibited quick bouts of central and peripheral positioning. Research by Sharpe and Hawkins (1992b) cited more expertise by a teacher who exhibited longer central positioning. Regarding the Modeling (W) element, the T-In exhibited this element much more frequently and in the chains. In other words, the T-In provided this element throughout the entire lesson and more often, while the T-Out exhibited this element primarily at the beginning of the lesson.

Considering the Off Task (R) element, the T-In exhibited this element for the same frequency (once), but for a longer duration than the T-Out. The length of this occurrence was due to the phone ringing in the gym and the teacher having to answer the call. This occurred for a constant duration of approximately forty-nine seconds. Regarding the Off Task (R) element, the T-In spent more time in this behavior.

The interview answer data also paralleled the elements exhibited by each teacher, reflecting a more instructional approach for the T-In and a less instructional approach by the TOut. The way that the T-In talked about his ongoing education was reflected in the way that he 
taught, regarding behaviors thought to be learned. The T-In expressed statements relating to the answer category of "relationship of master's degree and behavioral outcome," such as, “ it helped me refresh myself on a lot of things I had as an undergrad" (Line 10,11), and "it helped me refocus" (Line 13,14). Regarding the answer category of "coursework in master's education pertaining to behavior," the T-In stated that "fitness education courses" (Line 53) and "how to best deliver fitness education concepts" (Line 53, 54) were the most memorable elements he thought he had learned. Both of these categories of answers, regarding the way the T-In spoke about what he learned in his ongoing education, paralleled the instructional elements exhibited in the T-In behavioral system.

The way the T-Out talked about his ongoing education reflected how he taught as well. The T-Out expressed statements relating to the answer category of "relationship of master's degree and behavioral outcome," such as, "area of focus in behavior, um, management" (Line 10, 11) and "helps with many students that are off task" (Line 11). Regarding the answer category of "coursework in master's education pertaining to behavior," the T-Out stated, (students who were) "off task" (Line 43) and "more behavior, discipline to the higher end, they were physically" (unclear) (Line 43, 44) were the most memorable elements he thought he had learned. Both of these categories of answers, regarding the way the T-Out spoke about what he learned in his ongoing education paralleled a less instructional and more managerial elemental exhibition in the T-Out behavioral system.

Students. When examining the S-In and the S-Out elemental data, substantial differences emerged. The S-In exhibited a more content-focused behavioral system and a more predictable behavioral system. The S-Out exhibited a less content focused behavioral system and a less predictable behavioral system. The S-In exhibited more content learning elements in the 
descriptive data and in the chains. As stated by another researcher in the physical education field, the quality of one's instruction may be exhibited best by the product (R. Wiegand, personal communication, Fall, 2010). Consequently, the analysis of the content learning elements in this study was essential. In combined percentage, the content learning elements were higher for the T-In and lower for the T-Out, while the non-content learning elements were higher for the T-Out and lower for the T-In. Regarding the high frequency fulcrums, half of the fulcrums were content learning for the T-In, while the T-Out exhibited zero of these elements as fulcrums. Furthermore, the S-Out exhibited the Off Task (8) element as a fulcrum, while the T-In did not exhibit this element in his three-event, student sequence data. Regarding the sequence data, the S-In was more complex and yet more predictable for the content learning elements.

Prior research examining the behavioral systems of students has been absent regarding FSA. Research examining linear behaviors that are a proxy for student achievement, i.e. motor appropriate behavior, has suggested that more effective lessons exhibit higher percentages of this element, which assessed ALT-PE in this study (Hawkins, Wiegand \& Bahneman, 1983; McKenzie, 2009; Metzler, 1989; Parker, 1989). The motor appropriate behavior was higher for the S-In in this study, but both the S-In and S-Out could have exhibited higher percentages regarding this behavior. The S-In exhibited a motor appropriate percentage of $15.52 \%$, while the S-Out exhibited 7.30\%. Suggested percentages for ALT-PE, which was measured by motor appropriate behavior in this study, should equate to $10-20 \%$ or more class time to constitute an effective lesson (Metzler, 1989). With that said, some variance in percentage can occur relative to the instructional context in which the motor appropriate behavior occurs (A. Hawkins, personal communication, 2013). Regarding the S-In and the S-Out, the S-In lesson exhibited more effectiveness regarding motor appropriate behavior, but even the S-In lesson could have 
exhibited a higher percentage of this behavior to more fully denote what an effective lesson constitutes.

\section{Expertise Revisited}

In the physical education environment, comparisons, descriptions, lists, and summaries have been used in the past to describe what may constitute (Berliner, 1986; Dodds, 1994; Van der Mars et al., 1995; Sharpe \& Hawkins, 1992b). This system of analysis has frequently left researchers with a tabula rasa regarding a systematic modus operandi when analyzing expertise. Differing environments, organisms, and even students have complicated the replicability of such studies analyzing expertise. Analyzing subtle factors that occur in a temporal fashion may be the best method as of yet to attempt to analyze expertise in the physical education environment. Therefore, pedagogical researchers should have the tools to analyze what may constitute expertise and this may be accomplished by analyzing the behavioral field or system and everything that exists within it. An analysis pertaining to the environment and everything in it or FSA has been cited as a current holistic method to address this expertise conundrum.

\section{Research Implications}

Analyzing teachers by means of FSA in regards to their ongoing education and the insight learned from it may contribute to the development of more expert pedagogues. In examining the dimensions in this study, regarding the frequency and duration, rhythm, complexity, and coherence and velocity, differences were exhibited between the two teachers. In this instance, it was apparent that the T-In exhibited more expertise in his behavioral systems. What is also suspected is that the specific ongoing education obtained by each teacher played some part in the behavioral systems exhibited. The way that the T-In talked regarding the role of experience and discipline-specific ongoing education also came about when speaking of how 
expertise may have been gained. This avenue of focus should be followed when contributions to expertise in teaching are under examination. In analyzing these subtleties along with the interview data, the researcher can deduce what can be done to more fully develop future physical education teachers towards a definition of expertise.

Knowledge gained. Regarding this study, elements occurring temporally or in clusters, regarding the instructional elements for the teachers and content elements for the students, exhibited more expert practice. In this manner, expertise was exhibited for the T-In, amongst the systemic variables uncovered, by these specific instructional elements occurring in a more temporal pattern. Examples of elements that occurred in a temporal relationship with each other, or in more tightly packed clusters, which exhibited more expertise for the T-In were the Verbal Instruction: Skill Explanation (8) element and Teacher Questioning: Subject Matter (I) element; Verbal Instruction: Prompt (9) element and Specific Observation (T) element; and the Verbal Instruction: Strategies/ Rules (Q) element and Teacher Answers (U) element. These elements were not exhibited with as much temporal immediacy in the T-Out lesson. This study adds to the literature regarding expertise in physical education, in that relationships amongst instructional elements that have exhibited temporal contiguity specific to this environment can further delineate how certain elements should be exhibited in conjunction with each other to define what should constitute expertise. Expertise in this study regarding the T-In lesson was exhibited by a more complex and yet probable system that showed a relationship between instructional elements occurring with a high velocity, which has been detailed as being of high importance in previous literature (Sharpe \& Hawkins, 1992b). Regarding other studies, specifically using FSA, temporal contiguity amongst instructional elements was previously shown to exhibit expertise regarding the systemic variables under analysis. For example, the Sharpe and Hawkins (1992b) 
study elicited much of same results regarding expert versus novice instruction, as the expert exhibited "great fluency, complexity, rhythm, velocity, and coherence to a largely sequential instructional repertoire" (p. 70). Elements in this study, examining systemic variables, exhibited the same trend of high probability of high frequency elements regarding fewer dual chains occurring in the expert's lesson.

Regarding the student data, the S-In exhibited systemic variables occurring with more temporal contiguity regarding the content learning elements. Examples of systemic elements that occurred in a temporal manner with each other, or in more tightly packed clusters, which exhibited a more predicable system were the Motor Appropriate (1) element and the Cognitive: Passive (4) element; and the Cognitive: Passive (4) element and the Cognitive: Active (5) element. These elements were not exhibited with as much temporal immediacy in the S-Out lesson. When examining these elements in the student behavioral systems, not only were the percentages of content learning elements higher in the S-In lesson, but the temporal immediacy amongst these content learning elements occurred with a higher velocity and with more predictability. This may further purport the necessity for content learning elements to be occurring with temporal immediacy in conjunction with other elements that are conceptually related. The S-In lesson not only exhibited linear behaviors in higher percentages regarding the content learning elements, but also exhibited more expertise regarding the relationships occurring between specific content learning elements. These behavioral systems exhibited in this study can be used to further address what expert practice looks like from a student behavioral system standpoint.

Regarding both teacher and student data, this study adds to the expertise literature in that instructional elements in the teacher's behavioral system and the content learning elements in the 
student's behavioral system, should occur in a more temporal pattern, exhibiting a higher velocity of elements that have been shown in linear analysis alone to constitute effectiveness. In this manner, systemic variables that occur temporally can further denote what more expert practice looks like regarding a specific environment. This study has pinpointed specific elemental variables regarding a certain field and has determined that certain instructional elements and content learning elements should be exhibited and in conjunction with one another temporally to constitute more expert practice.

\section{Future Studies}

This study was set forth to discover the role that discipline-specific ongoing education may play in developing expertise. Although the results in this case study are not generalizable to other populations, FSA may be a viable tool in discovering expertise regarding other environments. By means of this analysis, it can be suggested that future studies be replicated by incorporating both teachers and their corresponding students to fully assess the environment in its entirety. Furthermore, the results of this study have provided a starting point in the analysis of discipline-specific ongoing education and teacher and student behavior in physical education. Replication of such studies in the future should be a necessity, and the analysis of both teacher and student behavior can more fully explain what is going on in the environment being analyzed. Characteristics of expert practice and what it constitutes can then be compiled by numerous studies analyzing these differing environments.

Teacher development. Training future teachers regarding more expert practice may be a method of teacher education, which in the past has not been addressed by simple linear behavioral percentage suggestions. Based on the behavioral systems in this study which exhibited more expertise, future teachers can be taught relationships that should exist between 
variables exhibited in this study and other pedagogical research examining expertise. In this manner, studies such as this one, which suggest that more expertise occurs with temporal relationships between the instructional elements for the teachers, i.e. skill explanations, teacher questioning, teacher answers, specifically observing, and how these behavioral interactions can address the student's behavior change, may be a method in the development of undergraduate and graduate teachers regarding expert practice. Educating these teachers in their educational preparation, in proper linear behaviors and behaviors in conjunction with each other which exhibit more expertise, can be accomplished by knowing what behavioral elements should occur with a higher velocity and in a more temporal fashion. Providing these future and/or current teachers with behavioral systems research that specifies which behaviors are instructional and which are not, as well as exhibiting which behaviors in conjunction with each other exhibit expert practice, would be a successful method of introducing this expert practice material.

Furthermore, these future teachers should be able to pinpoint what their students should be doing regarding their lessons, such as exhibiting a high degree of success within an activity and listening to content taught in a temporal fashion, so that they can address the lack thereof regarding their students' behavior. Behavioral systems research regarding the student behaviors can also be provided to these teachers, so that they can become cognizant as to what is and is not a content learning behavior. This analysis should occur from a standpoint of not only linear student behaviors, but also behaviors exhibiting temporal contiguity amongst the content learning elements that will assist in student achievement gains as exhibited in the example above. Based on this literature, knowing what to look for in oneself and in one's students could more successfully holistically train teachers as to what they and their students should be doing regarding an expert practice standpoint. 
Other areas of analysis. Another area in which analyzing expertise could be possibly examined regarding FSA, could be teachers who have obtained certification through the traditional route of teacher education programs versus alternative certification programs. Analyzing the behavioral systems of teachers who have gone through different types of certification processes such as these may unearth interesting differences by means of FSA. Gaining insight into what differences may exist between behavioral systems of teachers who have been trained differently, may provide further insight and relevance towards differences in even the type of teacher education programs completed.

Strengthening future studies. Regarding future studies using FSA to develop category systems based on the specific environment, incorporating another reviewer who is not in the physical education field to analyze the dialogue data and create a categorical system for the contextual and behavioral elements should be accomplished. In this manner, having a third researcher analyze whether the categories created exhibited what was actually evident in the dialogue could further control for the bias that may have occurred with only two reviewers. Incorporating a reviewer who is more objective regarding the data could possibly strengthen the reliability of the study. Having a reviewer who is not knowledgeable about the physical education field may further bring about concepts or observations not initially seen by the other two researchers.

Additionally, regarding the interview portion of this study, the creation of a richer study regarding the interview data could have been accomplished by means of a more structured interview analysis protocol suggested by prior literature regarding questionnaire creation and content analysis. Standardized, open-ended interviews could be used in a manner by which lines of questioning could be assessed by multiple reviewers before the questions are incorporated into 
the study. Three or more researchers could have been used to form a consensus as to what questions should have been used to gain insightful information regarding the type of ongoing education obtained by each participant in the study (Patton, 2002). Use of standardized, openended interviews, by means of properly developed questions, and analysis of text for reoccurring words or themes to see what "key phrases or concepts predominate," could be accomplished through this type of data analysis (Patton, 2002, p.453). In this manner, once key phrases or concepts are identified, convergent or divergent themes arising from these variables could be used to categorize the data by these researchers (Lincoln \& Guba, 1985; Patton, 2002). Once this step has been accomplished these researchers could then attest to the fact that the category system "fits" the data and that the data has been properly "fitted into" it (Patton, 2002, p.466). Afterwards, "interocular" differences (e.g. visible ones) in participant answers relative to these key phrases or concepts that have been categorized could be again properly disseminated (Patton, 2002, p.467). In this manner, a more structured, thorough analysis regarding multiple reviewers could have taken place to further legitimize what has been uncovered. In the future, using such analyses that incorporate a more structured form of questionnaire development and content analysis should be used to further legitimize how answers regarding specific questions were similar and different regarding the two participants in the study. 


\section{References}

Aaronson, D., Barrow, L., \& Sanders, W. (2003). Teachers and student achievement in the Chicago public high schools (Working Paper No. 2002-28). Chicago: Federal Reserve Bank of Chicago. Retrieved February 15, 2010, from http://www.chicagofed.org/webpages/publications/working_papers/2002/wp_28.cfm

Al-Mulla, A.F., (2002). Comparison of academic learning time in elementary school physical education classes taught by novice and experienced teachers in Bahrain. Journal of the International Council for Health, Physical Education, Recreation, Sport \& Dance, 38(1), 16-19.

Beighle, A., \& Pangrazi, R.P. (2002). The seven habits of highly effective physical education teachers. Teaching Elementary Physical Education, 13(4), 6-9.

Berliner, D.C. (1986). In pursuit of the expert pedagogue. Educational Researcher, 15(7), 5-13.

Betts, J.R., Zau, A.C., \& Rice, L.A. (2003). Determinants of Student Achievement: New Evidence from San Diego. Sacramento, CA: Public Policy Institute of California.

Brophy, J. (1982). Successful teaching strategies for the inner-city child. Phi Delta Kappan, 63, $527-530$.

Campbell, N.D., \& Lopez, E.J. (2008). Paying teachers for advanced degrees: evidence on student performance from Georgia. Journal of Private Enterprise, 24(1), 1-17.

Clotfelter, C.T., Ladd, H.F., \& Vigdor, J.L. (2007). Teacher credentials and student achievement in high school: A cross-subject analysis with fixed effects. Washington, DC: Urban Institute.

Cooper, J.O. (1981). Measuring behavior (2 ${ }^{\text {nd }}$ ed.) Columbus, OH: Charles E. Merrill. 
Cooper, J.O., Heron, T.E., \& Heward, W.L. (2007). Applied behavioral analysis (2 ${ }^{\text {nd }}$ ed.). Upper Saddle River, NJ: Pearson Education.

Covey, S. (1989). The seven habits of highly effective people. New York: Free Press.

Cruikshank, D.R. (1976). Synthesis of selected recent research on teacher effects. Journal of Teacher Education, 27(1), 57-60.

Dawe, H.A. (1984). Teaching: Social science or performing art? Harvard Educational Review, $54,111-114$.

Delprato, D.J. (1992). Behavioral field systems analysis: History and scientific relatives. Journal of Teaching in Physical Education, 12, 3-8.

Dodds, P. (1994). Cognitive and behavioral components of expertise in teaching physical education. Quest, 46, 153-163.

Dodds, P. \& Placek, J.H. (1991). Silverman's RT-PE review: Too simple a summary of a complex field. Research Quarterly for Exercise and Sport, 62(4), 365-368.

Eisner, E.W. (1983). The art and craft of teaching. Educational Leadership, 40, 4-13.

Fink, J., \& Siedentop, D. (1989). The development of routines, rules, and expectations at the start of the school year. Journal of Teaching in Physical Education, 8, 198-212.

Frome, P., Lasater, B., \& Cooney, S. (2005). Well-qualified teachers and high-quality teaching: Are they the same? (Research Brief). Atlanta, GA: Southern Regional Education Board.

Gage, N.L. (1978). The scientific basis of the art of teaching. New York: Teachers College Press.

Goe, L., \& Stickler, L. (2008). Teacher quality and student achievement: Making the most of recent research. Washington, DC: National Comprehensive Center for Teacher Quality.

Goldberger, M. (1984). Effective learning through a spectrum of teaching styles. Journal of Physical Education, Recreation \& Dance, 55(8), 17-21. 
Goldhaber, D.D., \& Brewer, D.J. (1998). When should we reward degrees for teachers? Phi Delta Kappan, 80(2), 134-138.

Goldhaber, D.D., \& Brewer, D.J. (2000). Does teacher certification matter? High school teacher certification status and student achievement. Education Evaluation and Policy Analysis, $22,129-145$.

Graham, G., \& Heimerer, E. (1981). Research on teacher effectiveness: A summary with implications for teaching. Quest, 33(1), 14-25.

Graham, G., Soares, P., \& Harrington, W. (1983). Experienced teachers' effectiveness with intact classes: An ETU study. Journal of Teaching in Physical Education, 2(2), 3-14.

Griffey, D.C., \& Housner, L. D. (1991). Differences between experienced and inexperienced teachers' planning decisions, interactions, student engagement, and instructional climate. Research Quarterly for Exercise and Sport, 62(2), 196-204.

Ha, A., Chan, D., \& Xu, L. (2002). Teacher behaviors and student activity levels in secondary school physical education. Journal of the International Council for Health, Physical Education, Recreation, Sport, and Dance, 39(3), 56-59.

Harris, D.N., \& Sass, T.R. (2007). What makes for a good teacher and who can tell? Paper presented at 2007 summer workshop of the National Bureau of Economic Research (NBER).

Harrison, J.M. (1987). A review of the research on teacher effectiveness and its implications for current practice. Quest, 39, 36-55.

Hastie, P. (1994). Selected teacher behaviors and student ALT-PE in secondary school physical education. Journal of Teaching in Physical Education, 13, 242-259. 
Hawkins, A. (1992). Preface: A personal introduction. Journal of Teaching in Physical Education, 12, 1-2.

Hawkins, A., Wiegand, R., \& Bahneman, C. (1983). The conceptual nature of ALT-PE and its use in an undergraduate teacher preparation program. Journal of Teaching in Physical Education, Monograph 1, 11-16.

Housner, L.D., \& Griffey, D.C. (1985). Teacher cognition: Differences in planning and interactive decision making between experienced and inexperienced teachers. Research Quarterly for Exercise and Sport, 56(1), 45-53.

James, A.R., \& Cruz, L.M. (2005). Address the "whole person": Ensuring student success. Teaching Elementary Physical Education. 16(6), 20-22.

Jensen, G. (2007). Expertise is more than an accumulation of experience. Magazine of Physical Therapy, 15(4), 55.

Johnson, K.A., (2000). The effects of advanced teacher training in education on student achievement. A Report of the Heritage Center for Data Analysis. (Publication number CDA00-09). Washington, DC: The Heritage Foundation.

Kantor, J.R. (1969). The scientific evolution of psychology (Vol. 2). Chicago: Principia Press.

Kounin, J.S. (1970). Discipline and group management in classrooms. New York: Holt, Rinehart \& Winston.

Lincoln, Y.S. \& Guba, E.G. (1985). Naturalistic inquiry. Newbury Park, CA: Sage Publications. McAvoy, R., Hursh, D.E., Nardi, A.H., Savage, L., \& Walls, R.T. (1988, June). The Psychology of Learning Team Benedum Foundation Hospital Proposal: Section II. (Available from Richard T. Walls, College of Human Resources and Education, West Virginia 
University, Research and Training Center, P.O. Box 6122, Morgantown, WV 265066122.

Metzler, M. (1989). A review of research on time in sport pedagogy, Journal of Teaching in Physical Education, 8, 87-103.

Monk, D.H. (1994). Subject area preparation of secondary mathematics and science teachers and student achievement. Economics of Education Review, 13(2), 125-145.

Pangrazi, R. P. (2001). Dynamic physical education for elementary school children (15 ${ }^{\text {th }}$. ed.) San Francisco, CA: Benjamin Cummings.

Parker, M. (1989). Academic Learning Time-Physical Education (ALT-PE), 1982 Revision. In P.W. Darst, D. B. Zakrajsek \& V. H. Mancini (Eds.), Analyzing Physical Education and Sport Instruction (pp. 195-206). Champaign, IL: Human Kinetics.

Pate, R.R., Davis, M.G., Robinson, T.N., Stone, E.J., McKenzie, T.L., \& Young, J.C. (2006). Promoting physical activity in children and youth: A leadership role for schools: A scientific statement from the American Heart Association Council on Nutrition, Physical Activity, and Metabolism (Physical Activity Committee) in collaboration with the Councils on Cardiovascular Disease in the Young and Cardiovascular Nursing, Circulation, 114, 1214-1224.

Patton, M.Q. (2002). Qualitative research and evaluation methods ( $3^{\text {rd }}$ ed.). Thousand Oaks, CA: Sage.

Placek, J. (1983). Conceptions of success in teaching: Busy, happy and good? In T. Templin \& J. Olson (Eds.), Teaching in physical education (pp. 46-56). Champaign, IL: Human Kinetics. 
Ray, R.D., \& Delprato, D.J. (1989). Behavioral systems analysis: Methodological strategies and tactics. Behavioral Science, 34(2), 81-127.

Rink, J.E. (2010). Teaching physical education for learning (6 ${ }^{\text {th }}$ ed.). New York, NY: McGrawHill.

Rivkin, S.G., Hanushek, E.A., \& Kain, J.F. (2005). Teachers, schools and academic achievement. Econometrica, 73(2), 417-458.

Roza, M., \& Miller, R. (2009). Separation of degrees: State-by-state analysis of teacher compensation for master's degrees. Schools in Crisis: Making Ends Meet. Center for Reinventing Public Education: Seattle, WA. Retrieved on February 15, 2011 http://www.crpe.org/cs/crpe/download/csr_files/rr_crpe_masters_jul09_db.pdf

Rubin, L.J. (1985). Artistry in teaching. New York: Random House.

Schempp, P., McCullick, B., St. Pierre, P., Woorons, S., You, J., \& Clark, B. (2004). Expert golf instructor's student teacher interaction patterns. Research Quarterly for Exercise and Sport, 75(1), 60-70.

Sharpe, T., \& Hawkins, A. (1992a). Strategies and tactics for field systems analysis. Journal of Teaching in Physical Education, 12, 9-23.

Sharpe, T., \& Hawkins, A. (1992b). Expert and novice elementary specialists: A comparative analysis. Journal of Teaching in Physical Education, 12, 55-75.

Sharpe, Jr., T.L., Hawkins, A., \& Wiegand, R. (1989). An analysis of model/practice versus verbal/rehearsal introductions of systems skills within an individually prescribed instructional system. Journal of Teaching in Physical Education, 9, 25-38.

Sharpe, T., \& Koperwas, J. (2010). BEST: Behavioral evaluation strategy \& taxonomy software. California: Sage. 
Siedentop, D.L. (2009). National plan for physical activity: Education sector. Journal of Physical Activity and Health, 6(Suppl 2), S168-S180.

Siedentop, D., Birdwell, D., \& Metzler, M. (1979, April). A process approach to measuring teacher effectiveness in physical education. Paper presented at the Annual Conference of the American Alliance for Health, Physical Education, Recreation and Dance, New Orleans.

Siedentop, D., \& Eldar, E. (1989). Expertise, experience, and effectiveness. Journal of Teaching in Physical Education, 8, 254-260.

Siedentop, D., Tousignant, M., \& Parker, M. (1982). Academic learning time-physical education 1982 revised coding manual. Unpublished manuscript, Ohio State University.

Silverman, S. (1991). Research on teaching in physical education. Research Quarterly for Exercise and Sport, 62(4), 352-364.

Terry, B.D. (2009). Shortchanging our kids: How poor teacher quality \& failed government policies harm students. Austin, TX: Texas Public Policy Foundation.

Thaxton, A.B., Rothstein, A.L., \& Thaxton, N.A. (1977). Comparative effectiveness of two methods of teaching P.E. to elementary school girls. Research Quarterly, 48(2), 420-427.

The Center for Public Education. (2005). Teacher Quality and Student Achievement: Research Review. Retrieved June $7^{\text {th }}, 2011$ from http://www.centerforpubliceducation.org/MainMenu/Staffingstudents/Teacher-quality-and-student-achievement-At-a-glance/Teacherquality-and-student-achievement-Research-review.html

U.S. Department of Health and Human Services. (2001). Healthy People 2010: Understanding 
and Improving Health. U.S. Government Printing Office, Washington, DC: U.S Government Printing Office.

U.S. Department of Education. (2004). No Child Left Behind Act 2001: A Toolkit for Teachers.

U.S. Department of Education (2011). Education resource organizations directory: State education agency. Retrieved from http://wdcrobcolp01.ed.gov/Programs/EROD/org_list.cfm?category_cd=SEA van der Mars, H., Darst, P.W., Vogler, E.W., \& Cusimano, B. (1995). Novice and expert physical education teachers: Maybe they think and decide differently...but do they behave differently? Journal of Teaching in Physical Education, 14, 340-347.

Vogler, E.W., van der Mars, H., Cusimano, B. E., \& Darst, P. (1992). Experience, expertise, and teaching effectiveness with mainstreamed and nondisabled children in physical education. Adapted Physical Activity Quarterly, 9, 316-329.

Walls, R.T., Nardi, A.H., von Minden, A.M., \& Hoffman, N. (2002). The characteristics of effective and ineffective teachers. Teacher Education Quarterly, 29(1), 39-48.

Webster, C.A. (2010). Increased student motivation through teacher communication: Six essential skills every physical educator should master. The Journal of Physical Education, Recreation, and Dance, 81(2), 1-64.

Werner, P., \& Rink, J. (1989). Case studies of teacher effectiveness in second grade physical education. Journal of Teaching in Physical Education, 8, 280-297.

Wenglinsky, H. (2000). How teaching matters: Bringing the classroom back into discussions of teacher quality (Policy Information Center Report). Princeton, NJ: Educational Testing Service. Retrieved February 15, 2011, from http://www.nocheating.org/Media/Education_Topics/pdf/teamat.pdf 
Zeng, H. Z., Leung, R. W., Liu, W., \& Hipscher, M. (2009). Physical education in urban high school class settings: Features and correlations between teaching behaviors and learning activities. Physical Educator.

Zeng, H.Z., Leung, R.W., \& Hipscher, M. (2010). An examination of teaching behaviors and learning activities in physical education class settings taught by three different levels of teachers. Journal of Social Sciences, 6(1), 18-28. 


\section{Appendix A}

\section{Teacher Behavior Categories}

\begin{tabular}{|c|c|c|}
\hline$\underline{\text { Teachers Behavioral Categories }}$ & $\underline{\text { Behavioral Code }}$ & $\underline{\text { Behavioral Definitions }}$ \\
\hline General Observation & 1 & $\begin{array}{l}\text { The teacher is watching } \\
\text { student groups or individuals } \\
\text { engaged in any category of } \\
\text { student behavior. The teacher } \\
\text { must not be engaged in any } \\
\text { other category or teacher } \\
\text { behavior in order to record } \\
\text { general observation. (non- } \\
\text { instructional) }\end{array}$ \\
\hline Positive Feedback: Specific & 2 & $\begin{array}{l}\text { The teacher makes a positive } \\
\text { verbal statement or gesture } \\
\text { following an appropriate } \\
\text { subject matter or non-subject } \\
\text { matter student behavior } \\
\text { clearly designed to increase or } \\
\text { maintain such responses in the } \\
\text { future. This statement is } \\
\text { directed to an individual } \\
\text { student. (instructional or }\end{array}$ \\
\hline
\end{tabular}




\begin{tabular}{|c|c|c|}
\hline & & managerial) \\
\hline Positive Feedback: General & 3 & $\begin{array}{l}\text { The teacher makes a positive } \\
\text { verbal statement or gesture } \\
\text { following an appropriate } \\
\text { subject matter or non-subject } \\
\text { matter student behavior } \\
\text { clearly designed to increase or } \\
\text { maintain such responses in the } \\
\text { future. This statement is } \\
\text { directed to more than one } \\
\text { student such as the class as a } \\
\text { whole or a group of students. } \\
\text { (instructional or managerial) }\end{array}$ \\
\hline Corrective Feedback: Skill & 4 & $\begin{array}{l}\text { The teacher makes a negative } \\
\text { or critical verbal statement or } \\
\text { gesture following an } \\
\text { inappropriate student behavior } \\
\text { regarding a skill clearly } \\
\text { designed to decrease such } \\
\text { responses in the future. } \\
\text { (instructional) }\end{array}$ \\
\hline Corrective Feedback: Negative Behavior & 5 & The teacher makes a negative \\
\hline
\end{tabular}




\begin{tabular}{|c|c|c|}
\hline & & $\begin{array}{l}\text { or critical statement or gesture } \\
\text { following an inappropriate } \\
\text { non-subject matter student } \\
\text { behavior in which they are not } \\
\text { listening to the teacher's } \\
\text { directions, are off task, or are } \\
\text { violating class rules. (non- } \\
\text { instructional) }\end{array}$ \\
\hline Management: General & 6 & $\begin{array}{l}\text { The teacher is engaged in } \\
\text { carrying out a non-subject- } \\
\text { matter task, in which they are } \\
\text { setting up equipment, turning } \\
\text { on the music or using any } \\
\text { equipment in a managerial } \\
\text { function. (managerial) }\end{array}$ \\
\hline Management: Direction & 7 & $\begin{array}{l}\text { The teacher is engaged in } \\
\text { carrying out a non-subject- } \\
\text { matter task, in which they are } \\
\text { directing students in a } \\
\text { managerial task. The teacher } \\
\text { may be giving students } \\
\text { options to assist them in }\end{array}$ \\
\hline
\end{tabular}




\begin{tabular}{|c|c|c|}
\hline & & $\begin{array}{l}\text { managerial tasks or are asking } \\
\text { questions of students engaged } \\
\text { in managerial portions of the } \\
\text { lesson. (managerial) }\end{array}$ \\
\hline Verbal Instruction: Skill Explanation & 8 & $\begin{array}{l}\text { The teacher is verbally } \\
\text { explaining aspects of a skill to } \\
\text { students, including how to do } \\
\text { the critical elements of a skill } \\
\text { or explaining how a skill will } \\
\text { be performed in a task or } \\
\text { activity. (instructional) }\end{array}$ \\
\hline Verbal Instruction: Prompt & 9 & $\begin{array}{l}\text { The teacher is verbally } \\
\text { prompting students or telling } \\
\text { them to do a skill at the } \\
\text { present moment. } \\
\text { (instructional) }\end{array}$ \\
\hline Verbal Instruction: Relating Statement & 0 & $\begin{array}{l}\text { The teacher is verbally } \\
\text { describing the skill in the } \\
\text { lesson and comparing it to } \\
\text { other skills, sports or activities } \\
\text { to create a deeper } \\
\text { understanding of the lesson }\end{array}$ \\
\hline
\end{tabular}




\begin{tabular}{|c|c|c|}
\hline & & $\begin{array}{l}\text { content. The teacher may be } \\
\text { describing how the content } \\
\text { may pertain to a larger area of } \\
\text { focus or future skill } \\
\text { development. (instructional) }\end{array}$ \\
\hline Verbal Instruction: Strategies/Rules & Q & $\begin{array}{l}\text { The teacher is verbally } \\
\text { explaining how individuals or } \\
\text { groups can be more effective } \\
\text { or competitive in an activity or } \\
\text { game performance. The } \\
\text { teacher may also be verbally } \\
\text { describing rules of activities, } \\
\text { games or skills. (instructional) }\end{array}$ \\
\hline Modeling & $\mathrm{W}$ & $\begin{array}{l}\text { The teacher demonstrates to } \\
\text { students how to do a subject } \\
\text { matter task, or participates } \\
\text { with students in a subject } \\
\text { matter task or activity. } \\
\text { Teacher may be using a } \\
\text { student (model) to help } \\
\text { demonstrate the proper critical } \\
\text { elements of a skill. }\end{array}$ \\
\hline
\end{tabular}




\begin{tabular}{|c|c|c|}
\hline & & (instructional) \\
\hline Physical Contact: Positive & $\mathrm{E}$ & $\begin{array}{l}\text { The teacher in engaged in a } \\
\text { positive manner with the } \\
\text { student, either physically } \\
\text { guiding a student towards } \\
\text { proper technique or keeping a } \\
\text { student on task through } \\
\text { positive contact. (instructional } \\
\text { or managerial) }\end{array}$ \\
\hline Off Task & $\mathrm{R}$ & $\begin{array}{l}\text { The teacher is not paying } \\
\text { attention to what are clearly } \\
\text { his or her responsibilities } \\
\text { regarding the class at hand. } \\
\text { (non-instructional) }\end{array}$ \\
\hline Specific Observation & $\mathrm{T}$ & $\begin{array}{l}\text { The teacher is watching one } \\
\text { student engaged in a subject } \\
\text { matter task for the purpose of } \\
\text { providing feedback related to } \\
\text { performance. The teacher } \\
\text { position must be proximal to } \\
\text { the student position so that } \\
\text { observation is clearly focused }\end{array}$ \\
\hline
\end{tabular}




\begin{tabular}{|l|l|l|}
\hline & & $\begin{array}{l}\text { on a specific student who is } \\
\text { performing. (instructional) }\end{array}$ \\
\hline Confusing Statement & Y & $\begin{array}{l}\text { The teacher makes a statement } \\
\text { that is contradictory, unclear } \\
\text { or not understood by the } \\
\text { students. (non-instructional) }\end{array}$ \\
\hline Teacher Answers & U & $\begin{array}{l}\text { The teacher answers a } \\
\text { student's subject matter } \\
\text { question during the lesson. } \\
\text { (instructional) }\end{array}$ \\
\hline Teacher Questioning: Subject Matter & I & $\begin{array}{l}\text { The teacher asks a student or a } \\
\text { group of students a question } \\
\text { regarding the subject matter. } \\
\text { (instructional) }\end{array}$ \\
\hline
\end{tabular}

\begin{tabular}{|l|l|l|}
\hline Contextual Categories & & \\
\hline$\underline{\text { Environmental Stimuli }}$ & Stimuli Code & Stimuli Definitions \\
\hline$\underline{\text { Context }}$ & & Opening activities which \\
Lesson Preview & O & $\begin{array}{l}\text { copare students for the lesson } \\
\text { content. (contextual) }\end{array}$ \\
\hline Instruction & P & Presentation and practice of \\
\hline
\end{tabular}




\begin{tabular}{|c|c|c|}
\hline & & $\begin{array}{l}\text { actual lesson content. } \\
\text { (contextual) }\end{array}$ \\
\hline Review & $\mathrm{A}$ & $\begin{array}{l}\text { Concluding activities of the } \\
\text { lesson. (contextual) }\end{array}$ \\
\hline Music & & \\
\hline On & $\mathrm{S}$ & $\begin{array}{l}\text { Music is turned On } \\
\text { (contextual) }\end{array}$ \\
\hline Off & $\mathrm{D}$ & $\begin{array}{l}\text { Music is turned Off } \\
\text { (contextual) }\end{array}$ \\
\hline Teacher Position & & \\
\hline Central & $\mathrm{F}$ & $\begin{array}{l}\text { The teacher is centrally } \\
\text { positioned in the gymnasium } \\
\text { regarding the group they are } \\
\text { observing, providing } \\
\text { feedback, instructing, or } \\
\text { managing. (contextual) }\end{array}$ \\
\hline Periphery & $\mathrm{G}$ & $\begin{array}{l}\text { The teacher is positioned on } \\
\text { the perimeter of the } \\
\text { gymnasium and not centrally } \\
\text { positioned, regarding the } \\
\text { group they are observing, }\end{array}$ \\
\hline
\end{tabular}




\begin{tabular}{|c|c|c|}
\hline & & $\begin{array}{l}\text { providing feedback, } \\
\text { instructing, or managing. } \\
\text { (contextual) }\end{array}$ \\
\hline \multicolumn{3}{|l|}{ Indiscriminate Stimuli } \\
\hline Transition & $\mathrm{H}$ & $\begin{array}{l}\text { The teacher changes the task } \\
\text { or activity within the lesson } \\
\text { prompting a smooth or } \\
\text { orderly transition. } \\
\text { (contextual) }\end{array}$ \\
\hline Indecision & $\mathrm{J}$ & $\begin{array}{l}\text { Teacher changes the task or } \\
\text { activity within lesson such } \\
\text { that a non-distinct or } \\
\text { disorderly transition takes } \\
\text { place. (contextual) }\end{array}$ \\
\hline \multicolumn{3}{|l|}{ Pupil Configuration } \\
\hline Warm-up & $\mathrm{K}$ & $\begin{array}{l}\text { The students are properly } \\
\text { positioned to participate in the } \\
\text { warm-up portion of the } \\
\text { lesson. (contextual) }\end{array}$ \\
\hline Center of Gym & $\mathrm{L}$ & $\begin{array}{l}\text { The students are positioned in } \\
\text { the center of the gym for } \\
\text { teacher directed instruction or }\end{array}$ \\
\hline
\end{tabular}




\begin{tabular}{|l|l|l|}
\hline & & $\begin{array}{l}\text { review of content. } \\
\text { (contextual) }\end{array}$ \\
\hline Drillwork & $Z$ & The students are properly \\
positioned to physically \\
participate in the content \\
portion of the lesson. \\
(contextual)
\end{tabular}


Appendix B

Student Behavior Categories

\begin{tabular}{|c|c|c|}
\hline $\begin{array}{l}\text { Students Behavioral } \\
\text { Categories }\end{array}$ & $\begin{array}{l}\text { Behavioral } \\
\text { Code }\end{array}$ & Behavioral Definitions \\
\hline Motor Appropriate & 1 & $\begin{array}{l}\text { The student is engaged in a subject matter } \\
\text { motor activity in such a way as to produce a } \\
\text { high degree of success. This includes skill } \\
\text { and/or health related fitness movements. } \\
\text { (content learning) }\end{array}$ \\
\hline Motor Inappropriate & 2 & $\begin{array}{l}\text { The student is engaged in a subject matter } \\
\text { motor activity but the task is either too difficult } \\
\text { for the individual's capabilities or is so easy } \\
\text { that practicing it could not contribute to lesson } \\
\text { goals. (non-content learning) }\end{array}$ \\
\hline Motor Supporting & 3 & $\begin{array}{l}\text { The student is engaged in a subject matter } \\
\text { motor activity the purpose of which is to assist } \\
\text { others to learn or perform the activity. (content } \\
\text { learning) }\end{array}$ \\
\hline Cognitive: Passive & 4 & $\begin{array}{l}\text { The student is appropriately involved in a } \\
\text { passive cognitive, subject matter task such as } \\
\text { watching or listening to the teacher describe }\end{array}$ \\
\hline
\end{tabular}




\begin{tabular}{|c|c|c|}
\hline & & content. (content learning) \\
\hline Cognitive: Active & 5 & $\begin{array}{l}\text { Verbal statements including asking or } \\
\text { answering questions related to lesson content. } \\
\text { (content learning) }\end{array}$ \\
\hline $\begin{array}{l}\text { On Task Management: } \\
\text { Verbal }\end{array}$ & 6 & $\begin{array}{l}\text { The student is appropriately engaged in verbal } \\
\text { statements, including asking or answering } \\
\text { questions, in carrying out an assigned non- } \\
\text { subject matter task. (managerial) }\end{array}$ \\
\hline $\begin{array}{l}\text { On Task Management: } \\
\text { Non-Verbal }\end{array}$ & 7 & $\begin{array}{l}\text { The student is appropriately engaged in non- } \\
\text { verbal behavior in carrying out an assigned } \\
\text { non-subject matter task. (managerial) }\end{array}$ \\
\hline Off Task & 8 & $\begin{array}{l}\text { The student is either not engaged in an activity } \\
\text { in which he or she should be engaged, or is } \\
\text { engaged in an activity other than the one in } \\
\text { which he or she should be engaged. (non- } \\
\text { content learning) }\end{array}$ \\
\hline Interim & 9 & $\begin{array}{l}\text { The student is engaged in a non-instructional } \\
\text { aspect of an ongoing activity. (non-content } \\
\text { learning) }\end{array}$ \\
\hline Waiting & 0 & $\begin{array}{l}\text { The student has completed a task and is } \\
\text { awaiting the next instructions or opportunity to } \\
\text { respond. (non-content learning) }\end{array}$ \\
\hline
\end{tabular}




\begin{tabular}{|l|l|l|}
\hline Confusion & W & $\begin{array}{l}\text { Student is visibly and/or verbally confused as to } \\
\text { what is going on in the lesson. (non-content } \\
\text { learning) }\end{array}$ \\
\hline Negative Self-Worth & E & $\begin{array}{l}\text { Student makes a comment by which they are } \\
\text { putting themselves down. (non-content } \\
\text { learning) }\end{array}$ \\
\hline
\end{tabular}

\begin{tabular}{|l|l|l|}
\hline Contextual Categories & & \\
\hline Environmental Stimuli & Stimuli Code & Stimuli Definitions \\
\hline Context & & $\begin{array}{l}\text { Opening activities which prepare students for } \\
\text { the lesson content. (contextual) }\end{array}$ \\
\hline Lesson Preview & O & Presentation and practice of actual lesson \\
content. (contextual)
\end{tabular}




\begin{tabular}{|c|c|c|}
\hline & & $\begin{array}{l}\text { observing, providing feedback, instructing, or } \\
\text { managing. (contextual) }\end{array}$ \\
\hline Periphery & $\mathrm{G}$ & $\begin{array}{l}\text { The teacher is positioned on the perimeter of } \\
\text { the gymnasium and not centrally positioned, } \\
\text { regarding the group they are observing, } \\
\text { providing feedback, instructing, or managing. } \\
\text { (contextual) }\end{array}$ \\
\hline \multicolumn{3}{|c|}{ Indiscriminate Stimuli } \\
\hline Transition & $\mathrm{H}$ & $\begin{array}{l}\text { The teacher changes the task or activity within } \\
\text { the lesson prompting a smooth or orderly } \\
\text { transition. (contextual) }\end{array}$ \\
\hline Indecision & $\mathrm{J}$ & $\begin{array}{l}\text { Teacher changes the task or activity within } \\
\text { lesson such that a non-distinct or disorderly } \\
\text { transition takes place. (contextual) }\end{array}$ \\
\hline \multicolumn{3}{|c|}{$\underline{\text { Pupil Configuration }}$} \\
\hline Warm-up & $\mathrm{K}$ & $\begin{array}{l}\text { The students are properly positioned to } \\
\text { participate in the warm-up portion of the lesson. } \\
\text { (contextual) }\end{array}$ \\
\hline Center of Gym & $\mathrm{L}$ & $\begin{array}{l}\text { The students are positioned in the center of the } \\
\text { gym for teacher directed instruction or review } \\
\text { of content. (contextual) }\end{array}$ \\
\hline Drillwork & $\mathrm{Z}$ & The students are properly positioned to \\
\hline
\end{tabular}




\begin{tabular}{|l|l|l|}
\hline & $\begin{array}{l}\text { physically participate in the content portion of } \\
\text { the lesson. (contextual) }\end{array}$ \\
\hline
\end{tabular}


Appendix C

Standardized open-ended interview questions and answers: Teacher Within-Field

1. What type of master's degree did you obtain?

Teacher In-F: I have my master's degree in physical education teacher education.

Interviewer: (Prompt) From?

Teacher In-F: From West Virginia University

2. What role do you feel your master's degree has played in your teaching behavior?

10

Teacher In-F: Um, the master's program and the degree, um, it helped me refresh myself on a lot of things I had as an undergrad. Um, it helped you know when you first get into a teaching position your first year you're just overwhelmed by everything that takes place and everything that you need to do that um by getting the master's degree it helped, it helped me refocus on you know the little things that you learn about, but you never really get to apply, until you have a job and sometimes those little things they get lost in the shuffle of being a first year teacher, so it helped, it helped me refresh on those things and you know, refine my teaching, um as a second, third, fourth year teacher, fifth year teacher now. Interviewer: Ok.

213 . Why did you choose to receive this type of education?

Teacher In-F: I..... 
Teacher In-F: I always, um, well ongoing wise, um, there's not a whole lot offered out $\underline{\text { there for, ah, specialists, like art, phys. ed., music, that kind of stuff and I didn't want to }}$ go and sit through graduate classes for things that weren't necessarily going to pertain to $\underline{\text { my every day, ah, teaching behavior, so that was why I decided to go into the route of }}$ phys. ed., um, so that I could use learn and use applications right in my classes.

Interviewer: Ok.

4. How do you feel you would evaluate you teaching behavior before your master's degree? Teacher In-F: Um, before my master's degree, I think I was a decent teacher, decent first yah know new teacher. Um, like I said earlier, the master's program in phys. ed. helped $\underline{\text { me realize some things that I should have been doing and wasn't doing and it helped me, }}$ $\underline{\text { um refine my skills a little bit better, gave me some practical application to use in }}$ different situations, um, so, you know it, it, it helped in my delivery of physical education, um, information and content.

5. Ok then how do you feel you would evaluate your teaching behavior after your master's degree then?

Teacher In-F: I think, yah know, it's much better not only because of the master's program it helped me realize some things, but also just experience alone, um, I think that $\underline{\text { experience is very valuable and um that experience in conjunction with the master's }}$ degree in phys. ed. has really just helped my teaching, ah helped my students learn better, helped them understand things better and ah it's been, it's been very rewarding for both myself and the students I teach. 
6. Ok was there specific coursework in your master's degree that focused on proper teacher and student behavior in the teaching environment?

Teacher In-F: Yes, ah

Interviewer: (Prompt) Which behaviors?

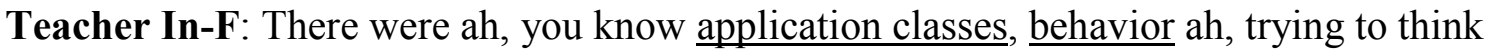
back to the ah, behavioral science type courses, um, fitness education courses, $\underline{\text { how to }}$ best deliver fitness education concepts, um, classes dealing with special education $\underline{\text { students, how to best approach teaching them. Um, just you know, a whole, a whole }}$ gambit of things that basically, you know teaching is dealing with behavior, so you know anything that had to deal with that, with teaching has to do with behavior of the student and behavior of yourself teaching those students, so you know many, many of the classes, $\underline{\text { although informative also came back to the behavioral end of things and, and, ah, tied }}$ everything together.

7. Ok so what was the reason you decided to obtain a master's degree in the first place? advancement? way things are here in the state of Pennsylvania right now, ah, the more education you have the more money you get paid, um, so you know knocking out two birds with one $\underline{\text { stone, trying to become a better teacher, while also you know trying to earn some extra }}$ 

were the main motivation for obtaining my master's degree.

Interviewer: Ok, thank you very much. 
Appendix D

1. What type of master's degree did you obtain and from?

Teacher Out-F: I obtained a master's administration, ah, educational leadership and it was from, ah, the University of Edinboro.

2. Ok what role do you feel your master's degree has played in your teaching behavior? Teacher Out-F: Um, with, ah, educational leadership it allowed me to be able to help the $\underline{\text { students, }}$ um, with delegation of roles and also I had, ah an area of focus in behavior, um, $\underline{\text { management, which helps with many students that are off task or that, um, need a little }}$ $\underline{\text { bit of extra, um, encouragement, um, with different, ah, behaviors and discipline }}$ situations that arise.

Interviewer: Ok.

3. Why did you choose to receive this type of education, ongoing?

Teacher Out-F: First of all I thought it would be a good career choice, um, for, um, obtaining some type of administration role in a school district and also allow me to pursue other, um, areas in sports related to, um, athletic director or head of a department. degree? 
Teacher Out-F: Um I would say it's very similar, ideas, roles, $\underline{\text { same type of way I taught }}$ class with ah curriculums the main difference I used was, um, like I said I focused more on delegating roles to my students, um because I feel that some students interact very well with other students, I picked that up with behavior, um behaviors, and also researching different types of behaviors.

5. How do you feel you would evaluate your teaching behavior after your master's degree then? Teacher Out-F: Um, I feel it's helped, I feel it's helped significantly just also going through the process of getting my masters in that cause I feel that, um, ah that, it end all um, I'm happy with the decisions I did, so it helped me in my class work that I feel a positive manner toward my students that I'm molding them into a better, ah, productive student.

6. Ok ah, was there specific coursework in your master's degree that focused on proper teacher and student behavior in the teaching environment?

Teacher Out-F: Ah, a few, more like focusing on behavior.

Interviewer: (Prompt) Yah, which, which behaviors maybe? behaviors and ways to pinpoint them regarding um, ah, students that were attention seeking or off task or some that I wasn't really aware of, more behavior, discipline to the higher end, they were physically, I wasn't sure what to do with them and now, um, found out more of the ball. 
7. Ok ok, what was the reason you decided to obtain a master's degree in the first place? Was it permanent certification, self improvement, professional advancement? Teacher Out-F: Professional advancement, also um, it was I needed to like you said pretty much all of them, professional advancement was the main one and then also, ah, I had to continue with my certification. 
Appendix E

Consent Letter Teachers

\title{
WestVirginiaUniversity. \\ Office of Research Compliance
}

\section{CONSENT AND INFORMATION FORM}

\author{
Teacher's OMR ICF
}

Principal Investigator: Hawkins, Andrew

Department: Physical Education

Tracking Number: $\quad \mathrm{H} \cdot 23978$

Study Title:

An Exploration of Master's Degree Field Study and Teacher and Student Behavior in P.E.

Co-lnvestigator(s):

Davis, William,

Sponsor

N/A

\section{Contact Persons}

In the event you experience any side effects or injury related to this research study, you should contact Dr. Hawkins at 304/293-0849. (After hours contact Mr. William Davis at 724/986-9674)If you have any questions, concerns, or complaints about this research study, you can contact Dr. Andrew Hawkins or Mr. William Davis at 304/293-0849 or 724/986-9674 respectively.

For information regarding your rights as a research subject, you may contact the Office of Research Compliance at 304/293-7073.

\section{Introduction}

In addition if you would like to discuss problems, concerns, have suggestions related to research, or would like to offer input about the research, contact the Office of Research Integrity and Compliance at 304293-7073.

Tracting 2 Hage 1 of 5


You, , have been asked to participate in this research study, which has been explained to you by Mr. William Davis, M.Ed., ABD. This research study is being conducted by Mr. William Davis in the Department of Physical Education at West Virginia University. This research study is being conducted to fulfill the requirements for a doctoral dissertation in Kinesiology in the Department of Physical Education at West Virginia University, under the supervision of Dr. Andrew Hawkins.

\section{Purposes of the Study}

The purpose of this research study is to learn more about teacher and student behavior and how ongoing education may play a part in developing better practice in physical education. WVU expects to enroll approximately 50 subjects; a total of approximately 50 subjects at all sites are expected to participate in this research study.

\section{Description of Procedures}

This research study involves the video recording of you and your student's behavior in three regularly occurring physical education lessons. Extraction from the video recordings of you and your student's behavior in relation to your ongoing education in physical education will be evaluated and compared to another physical education teacher and his students who has obtained differing ongoing education. I, Mr. William Davis, will video record you and your student's behavior to see if similarities and/or differences exist between behavior exhibited in your lesson and the lesson of the other physical educator, Behavior of the teacher that may be extracted and analyzed are as follows: encouragement, positive feedback, corrective feedback, modeling, etc. Behavior of your students that may be extracted and analyzed may be as follows! activity appropriate behavior, activity inappropriate behavior, student waiting, student off-task behavior, etc. Each of these behaviors has been previously exhibited in the physical education environment. These are examples of behavior that may be extracted from the videos to examine differences in you and your student's behavioral sequences in relation to your instruction. It will take approximately three video recorded lessons for its completion. You will be recorded for three lessons and you will pick the lesson you feel is your "best teach" to be analyzed. Your students will be video recorded in two

\begin{tabular}{|c|c|}
\hline Trackding $*$. & H.23978 \\
\hline Apperoved Ore & $06 / 076012$ \\
\hline Valid Throught & 060600013 \\
\hline Lent Amended. & NA \\
\hline
\end{tabular}

$\overline{\text { Initals }} \overline{\text { Deth }}$


Tracking * H.29978

minute intervals throughout the lesson to get a representative sample of their behavior regarding your instruction. After selection of the video recorded lesson you feel is your "best teach", you will be asked a series of questions that relate to the ongoing education you have obtained.

\section{Risks and Discomforts}

There are no known or expected risks from participating in this research study, except for being uncomfortable with the video recordings taking place within the gymnasium. If you are uncomfortable with the video recordings, you can be removed from the video recorded lessons and consequently the research study at any time.

\section{Alternatives}

You do not have to participate in this study.

\section{Benefits}

You may not receive any direct benefit from this study. The knowledge gained from this study may eventually benefit others.

\section{Financial Considerations}

There are no special fees for participating in this study.

If you choose to participate in this study, you will receive $\$ 100.00$ after audio and video recording is completed.

\section{Confidentiality}

Any information about you that is obtained as a result of your participation in this research will be kept as confidential as legally possible.

Audio and video recordings will be kept locked up and will be destroyed as soon as possible after the research is finished.

In any publications that result from this research, neither your name nor

\begin{tabular}{|c|c|c|c|c|}
\hline Tracking $*$ : & H.23978 & Page 3 of 5 & & \\
\hline Appiovied Ort & $06 / 076012$ & & Initals & $\overline{\text { Daten }}$ \\
\hline Vald Through: & $06 / 06 / 2013$ & & & \\
\hline Last Amended: & NIA & & & \\
\hline
\end{tabular}


Tracking a: H-23978

any information from which you might be identified will be published without your consent.

\section{Voluntary Participation}

Participation in this study is voluntary. You are free to withdraw your consent to participate in this study at any time. In the event new information becomes available that may affect your willingness to participate in this study, this information will be given to you so that you can make an informed decision about whether or not to continue your participation. You have been given the opportunity to ask questions about the research, and if you need further explanation to not hesitate to call me, Mr. William Davis, at 724/986-9674.

\begin{tabular}{|c|c|c|}
\hline Tracking 2 : & $\mathrm{H}-23978$ & Page 4 of 5 \\
\hline Appioved Ort & $06 / 070012$ & \\
\hline Vald Throughe & $06 / 06 / 2013$ & \\
\hline Lat Amended: & NA & \\
\hline
\end{tabular}


Upon signing this form, you will receive a copy.

I willingly consent to participate in this research.

Blanabure of Bubject or Frinited Name

Qubjects Lepal Represertatys

The participant has bad the opporumity to have questions addressed. The participant willingly agrees to be in the study.

Blanature of Investgater or co-Investpeor
Frinied Name
TIme

\begin{tabular}{|c|c|c|}
\hline Tracting 5 & $\mathrm{H}-2957 \mathrm{~s}$ & Page 5 of 5 \\
\hline Apponid Gre & acongots & \\
\hline Yald Throught & achegots & \\
\hline Lent Emandad: & NuE & \\
\hline
\end{tabular}


Appendix F

Consent-Assent Letter Parents/Students

\title{
W. WestVirginiaUniversity. \\ Office of Research Compliance
}

PARENTAL OR GUARDIAN CONSENT AND INFORMATION FORM

\author{
OMR-Parental, Guardian, Assent Consent
}

Principal Investigator: Hawkins, Andrew

Department: Physical Education

Tracking Number: $\quad \mathrm{H}-23978$

\section{Study Title:}

An Exploration of Master's Degree Field Study and Teacher and Student Behavior in P.E.

\section{Co-Investigator(s):}

Davis, William

\section{Sponsor}

N/A

\section{Contact Persons}

In the event you experience any side effects or injury related to this research study, you should contact Dr. Hawkins at 304/293-0849. (After hours contact Mr. William Davis at 724/986-9674)If you have any questions, concerns, or complaints about this research study, you can contact Dr. Andrew Hawkins or Mr. William Davis at 304/293-0849 or 724/986-9674 respectively.

For information regarding your rights as a research subject, you may contact the Office of Research Compliance at 304/293-7073.

\section{Introduction}

In addition if you would like to discuss problems, concerns, have suggestions related to this research study, or would like to offer input about this research study, contact the Office of Research Integrity and Compliance at 304-293-7073.You, asked to allow your child , are being , to participate in this

\begin{tabular}{|c|c|c|}
\hline Tracking * : & H.23978 & Page 1 of 5 \\
\hline Appiovied Ore & $06 / 076012$ & \\
\hline Vald Through & 0610600013 & \\
\hline Last Amended: & NA & \\
\hline
\end{tabular}

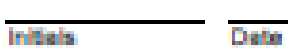


Tracking a: H-23978

research study, which will be explained to you by Mr. William Davis, M.Ed., ABD. This research study is being conducted by Mr. William Davis in the Department of Physical Education at West Virginia University. This research study is being conducted to fulfill the requirements for a doctoral dissertation in Kinesiology in the Department of Physical Education at West Virginia University, under the supervision of Dr. Andrew Hawkins.

\section{Purposes of the Study}

The purpose of this research study is to learn more about teacher and student behavior and how ongoing education may play a part in developing expertise in physical education. WVU expects to enroll approximately 50 subjects; a total of approximately 50 subjects at all sites are expected to participate in this research study.

\section{Description of Procedures}

This research study involves the video recording of your child, their classmates and their corresponding physical education teacher in three physical education lessons. This video recording will occur in their regularly scheduled physical education classes. Teachers and students in this setting will be video recorded to find out if their behaviors are similar or different when compared to another physical education teacher's lesson. I, Mr. William Davis will record your child 's teacher's behavior and your child's behavior to see how your child 's teacher's behavior affects your child's behavior. In this manner, I can then examine the similarities and differences in both the teacher's behavior and their student's behavior in relation to each of the two teacher's ongoing educations. Behaviors of the teacher that may be analyzed are as follows: encouragement, positive feedback, corrective feedback, modeling, etc. Behaviors of your child in relation to the teacher's behavior that may be analyzed may be as follows: activity appropriate behavior, activity inappropriate behavior, student waiting, student off-task behavior, etc. Each of these behaviors has been exhibited in the physical education environment previously. These are examples of behaviors that may be extracted from the videos to examine differences in your child 's teacher's behavior and your child 's behavior. Your child 's behavior may be in response to their teacher's behavior, therefore your child will not be identified specifically by name in this study. It will take approximately three video recorded lessons for its completion.

\begin{tabular}{|c|c|c|c|c|}
\hline Tracting $z$ : & $\mathrm{H} \cdot 23078$ & Page 2 of 5 & & \\
\hline $\begin{array}{l}\text { Apporoved Ore } \\
\text { Valid Throught }\end{array}$ & $\begin{array}{l}06 / 076012 \\
06 / 06 / 2013\end{array}$ & & Inites & $\overline{\text { Dater }}$ \\
\hline Last Amendod. & NA & & & \\
\hline
\end{tabular}


Tracking a: H-23978

Your child and their classmates will be video recorded in two minute intervals throughout the lesson to get a representative sample of their behavior regarding their teacher's instruction.

\section{Risks and Discomforts}

There are no known or expected risks from participating in this study, except for being uncomfortable with the video recording taking place within the gymnasium. If your child is uncomfortable with the video recording, they can be removed from the video recorded lessons and consequently the study at any time.

\section{Alternatives}

Your child does not have to participate in this study.

Alternatives that could be considered in your child's case include participation in physical education activities during this time period in which your child is not being video recorded. I, Mr. William Davis, will have a trained physical education teacher from the West Virginia University Physical Education Teacher Education Department on site with me during the video recorded physical education lessons to teach a physical education lesson to students who do not want to be video recorded during the research study. This lesson will be of the same content that the physical education teacher at your child's elementary school will be teaching that day.

\section{Benefits}

Your child may not receive any direct benefit from this study. The knowledge gained from this study may eventually benefit others.

\section{Financial Considerations}

No costs will be incurred to your child for participation in the study.

\section{Confidentiality}

Any information about your child that is obtained as a result of participation in this research study will be kept as confidential as legally possible. Audio or video recordings will be kept locked up and will be destroyed as soon as possible after the research study is finished. In any

\begin{tabular}{|c|c|c|c|c|}
\hline Tracking 2 : & H.23978 & Page 3 of 5 & & \\
\hline Approvid Ort & $06 / 076012$ & & Initals & $\overline{\text { Date }}$ \\
\hline Vald Through & $06 / 06 / 2013$ & & & \\
\hline Lest Amended: & NA & & & \\
\hline
\end{tabular}


Tracking at H-23978

publications that result from this research study, neither your child 's name nor any information from which your child might be identified will be published without your consent.

\section{Voluntary Participation}

Participation in this research study is voluntary. Your child may refuse to participate in this research study. Your child may withdraw from this research study at any time. Refusal to participate or withdrawal will not affect your child's grades and will involve no penalty to your child. In the event new information becomes available that may affect your willingness to allow your child to participate in this research study, this information will be given to you so that you can make an informed decision about whether or not to continue your child's participation. If you have read what will occur in the research study and have any questions and/or need any clarification as to what will take place before you will allow your child to participate in this research study please do not hesitate to call me, Mr. William Davis at 724/986-9674. Assent:1. I have explained all aspects of the research to the subject to the best of his or her ability to understand.2. I have answered all the questions of the subject relating to this research. 3 . The subject agrees to be in the research. 4 . I believe the subject's decision to enroll is voluntary. Conducting Assent Discussion
Parent/GuardianMy child appears to understand the research to the best of Signature of Person his or her ability and has agreed to participate.

Signature of

Parent/Guardian Date

\begin{tabular}{|c|c|c|c|c|}
\hline Tracking 2 : & H-23978 & Page 4 of 5 & & \\
\hline Approved Ort & $\begin{array}{l}06 / 076012 \\
0606 / 2013\end{array}$ & & Initals & $\overline{\text { Dath }}$ \\
\hline $\begin{array}{l}\text { Valid Through: } \\
\text { Lent Amended: }\end{array}$ & & & & \\
\hline
\end{tabular}


Tracting at $\quad$ H.29978

Upon signing this form, you will receive a copy.

I willingly consent to allow my child to participate in this research.

SIgnature of Parent or Guardan

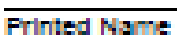

Date Time

The parent/guardian has had the opportunity to have questions addressed. The parent/guardian willingly agrees to allow his/ber child to be in the study.

SIgnature of Inveatigator or

Printed Name

Date

Time

Co-inveatgator

Tracking \&: $\quad H-23978$

Approved Or. 06070012

Valid Throughe OS/06/2013

Last Amended: NUA
Page 5 of 5

Iniliels

Dete 